\title{
Survey of ANL Organization Plans for Word Processors, Personal Computers, Workstations, and Associated Software
}

\author{
By
}

Karen R. Fenske

Viki S. Rockwell

August 1992 


\section{ACKNOWLEDGMENTS}

We thank the members of the various ANL organizations who completed the informational survey forms. We also thank Mike Boxberger, Fred Moszur, James Regula, and Dennis Tussing for contributing to and reviewing the Survey. Special thanks goes to John Jasunas for his patience, perseverance, and assistance in collecting and organizing the individual survey information.

We have created this document with Microsoft Word on the Apple Macintosh for printing on the Linotype L300P typesetter. 


\section{HOW TO USE THE SURVEY}

The Computing and Telecommunications Division (CTD) has compiled this Survey of ANL Organization plans for Word Processors. Personal Computers, Workstations, and Associated Soj"ware (ANL/TM, Revision 4) to provide DOE and Argonne with a record of recent growth in the acquisition at d use of personal computers, microcomputers, and word processors at ANL. Laboratory planners, service providers, and people involved in office automation may find the Survey useful. It is for internal use only, and any unauthorized use is prohibited.

Readers of the Survey should use it as a reference document that (1) documents the plans of each organization for office automation, (2) identifies appiopriate planners and other contact people in those organizations, and (3) encourages the sharing of this information among those people making plans for organizations and decisions about office automation. The Survey supplements information in both the ANL Statement of Site Strategy for Computing Workstations (ANL/TM 458) and the ANL Site Response for the DOE Information Technology Resources Long-Range Plan (ANL/TM 466).

CTD's procedure for compiling this Survey has been to request that each organization provide the following information in previously formatted tables:

- Contact person(s), subject, phone number, and c-mail address.

- Numbers of personal computers and workstations that existed in FY 1991, that exist in FY 1992 and that will exist in FY1993.

- Numbers of word processors that existed in FY 1991, that exist in FY1992, and that will exist in FY1993.

- Names and numbers of software packages that will be in use by September 1992.

- Numbers of network connections that exist in FY1992 and that will exist in FY1993.

- Additional plans for office automation.

If a cost center did not provide information for a table, we omitted that table from the Survey.

"N/A" symbolizes a number that a cost center did not provide in a completed table.

The following organizations did not participate in FY1993:

- Argonne West--EBR-II (Cost Center 102)

- Argonne West--Radiation Monitoring (Cost Center 551)

- Argonne West--Safety Staff (Cost Center 536)

- Argonne West--Special Materials (Cost Center 532)

Use the appropriate prefix for your site to call extensions listed for individual contacts. 
Advanced Photon Source (Cost Center 130) ........................................................................................... 1

Argonne East-Experiment Analysis (Cost Center 104) .......................................................................

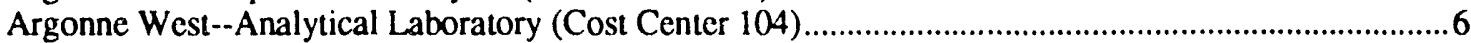

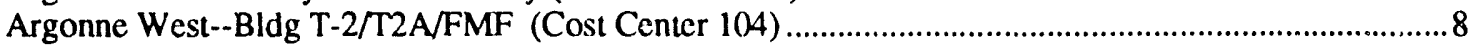

Argonne West--Communications and Computing Services (Cost Center 552) .........................................11

Argonne West-- Fuel Cycle (Cost Center 118) ..................................................................................14

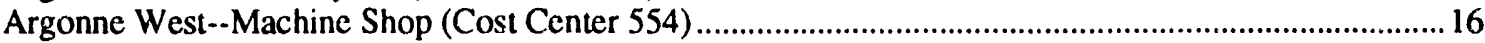

Argonne West-..Office of Operational Safety (Cost Center 570) …......................................................... 18

Argonne West--Office of Quality Assurance (Cost Center 102) .....................................................................20

Argonne West--Plant Services (Cost Center 557) ......................................................................................22

Argonne West Reactor Program Services Division--Accounting (Cost Center 533) ...................................24

Argonne West Reactor Program Services Division--Division Office (Cost Center 530) ...........................26

Argonne West Reactor Program Services Division--Environmental Waste Management (Cost

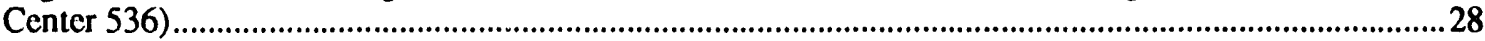

Argonne West Reactor Program Services Division-- Food Services (Cost Center 559).............................. 30

Argonne West Reactor Program Services Division--Human Resources (Cost Center 531) ........................31

Argonne West Reactor Program Services Division--Information Services (Cost Center 537)...................33

Argonne West Reactor Program Services Division-.-Prucurement (Cost Center 534) ...............................35

Argonne West Reactor Program Services Division--Radiation Fire Safety (Cost Center 551) ..................36

Argonne West Reactor Program Services Division--Receiving and Inspection (Cost Center 561) ............38

Argonne West Reactor Program Services Division--Safeguards (Cost Center 532) ...................................40

Argonne West Reactor Program Services Division--Security (Cost Center 535) ..........................................42

Argonne West Reactor Program Services Division--Supply (Cost Center 538) ........................................44

Argonne West--Site Engineering/Project Management (Cost Center 556) ...............................................46

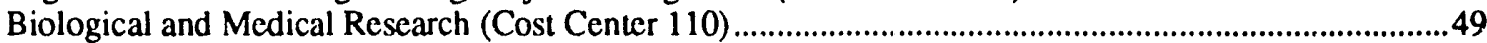

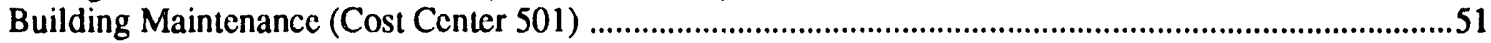

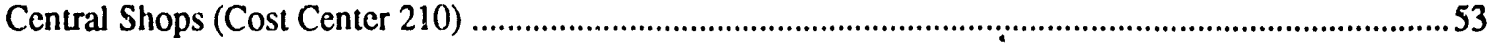

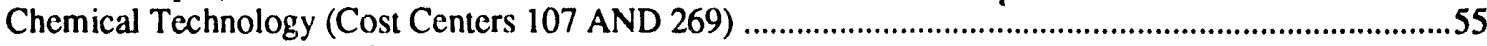

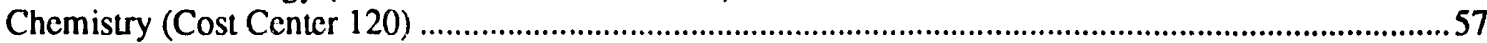

Chief Operations Officer (Cost Center 202) ...........................................................................................61

Computing and Telecommunications Division (Cost Centers 146, 245, 247, and 296)............................63

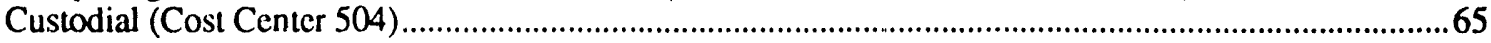

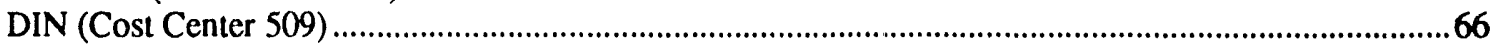

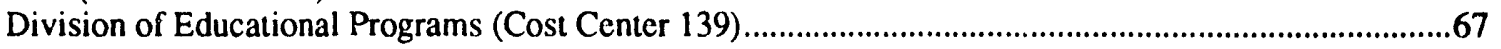

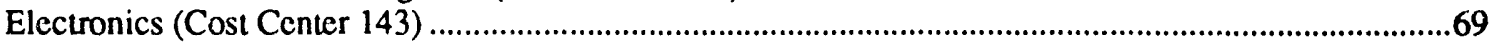

Energy, Environmental, and Biological Research Program Administration (Cost Center 274) ..................71

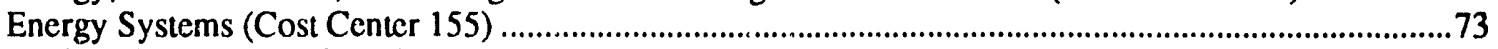

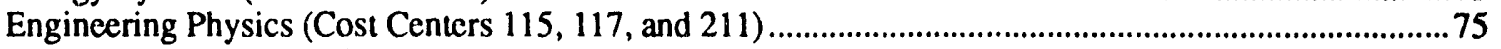

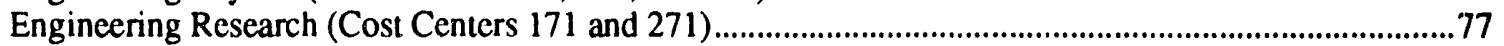

Environment, Safety, and Health/Quality Assurance Oversight (Cost Center 333) .....................................79

Environment, Safety, and Health Division-Division Office (Cost Center 235) ..........................................81

Environment, Safety, and Health Division-Dosimetry and Analytical Services (External

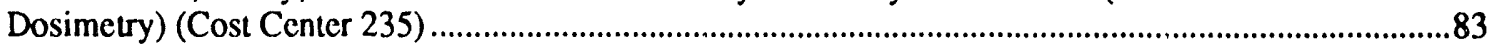

Environment, Safety, and Health Division-Dosimetry and Analytical Services (Internal Dosimetry)

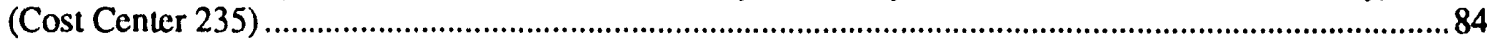

Environment, Safety and Health Division--Emergency Management (Cost Center 235) ............................86

Environment, Safety and Health Division--Fire Protection (Cost Center 235) ..........................................88

Environment, Safety, and Health Division-Health Physics (Cost Center 235) ...........................................90

Environment, Safety, and Health Division-Industrial Hygiene (Cost Center 235)....................................93

Environment, Safety, and Health Division-Safety Engineering Section (Cost Center 235) .......................95

Environment, Safety, and Health Division-Training and Quality Assurance (Cost Center 235) .................97

Environmental Assessment and Information Sciences Division (Cost Center 165) ....................................99

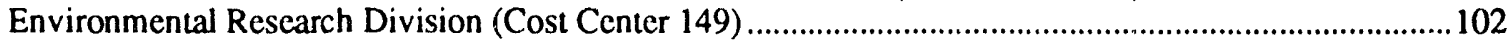

Environment and Waste Management Program (Cost Center 140) ......................................................... 105

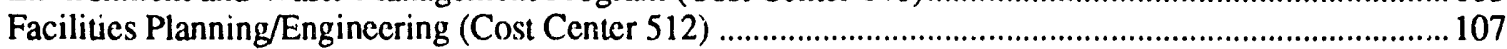

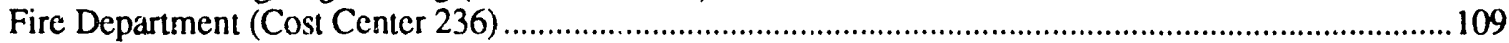

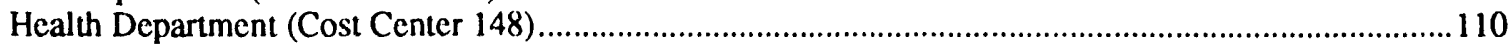

High Energy Physics (Cost Center 137) .........................................................................................112 


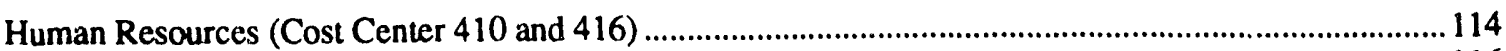

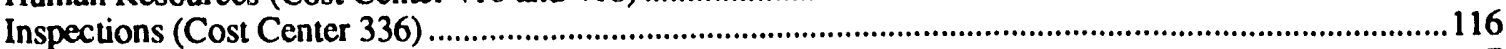

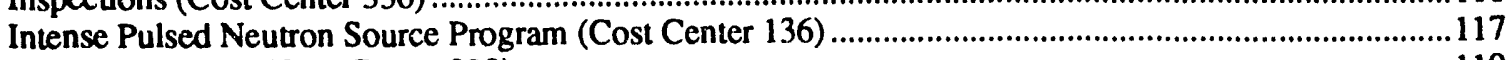

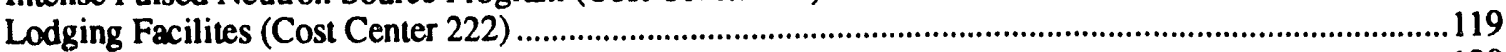

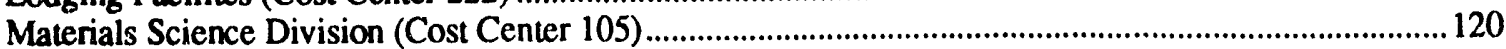

Materials and Components Technology (Cost Center 114) ........................................................................... 122

Materials and Services Department (Cost Center 315) .................................................................................124

Mathematics and Computer Science (Cost Center 145) .............................................................................126

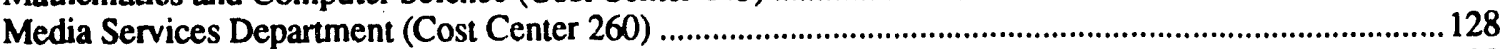

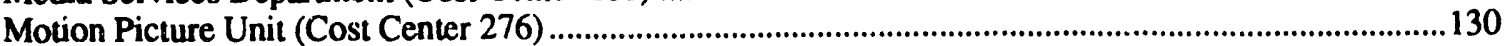

Office of the Chief Financial Officer (Cost Centers 400, 401, 402, and 403) .............................................132

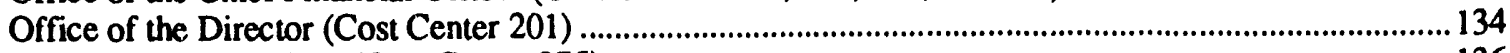

Office of Public Affairs (Cost Center 275) .........................................................................................136

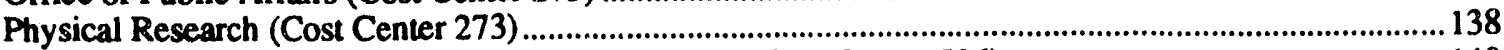

Plant Facilities and Services--Plant Manager's Office (Cost Center 506) ....................................................140

Procurement Department (Cost Center 322) .................................................................................................142

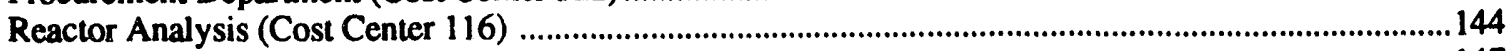

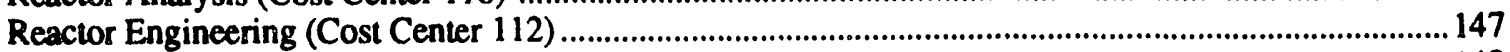

Secretarial and Clerical Services (Cost Center 416) ....................................................................................149

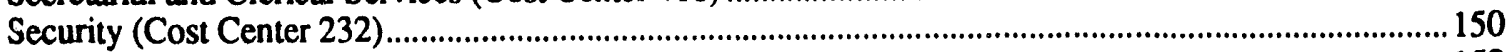

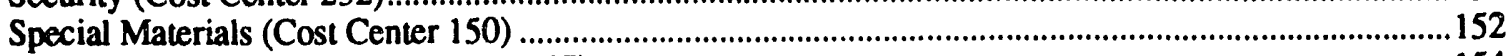

Special Projects Office (Cost Center 197) .............................................................................................. 154

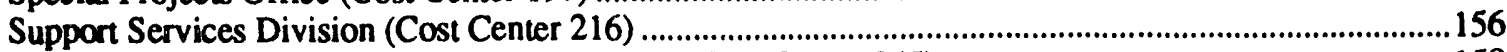

Technical Communications Service Department (Cost Center 265) ........................................................ 158

Technical Information Services Department (Cost Center 161) ......................................................................161

Travel Office (Cost Center 319) ...................................................................................................................163

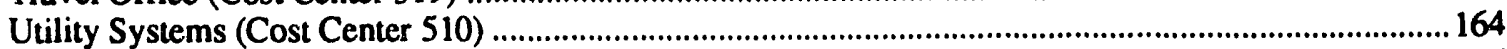

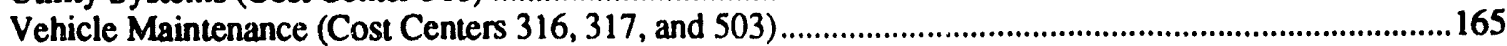

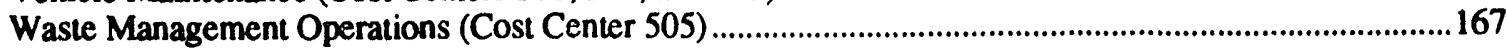




\section{Advanced Photon Source (Cost Center 130)}

\begin{tabular}{|ccccc|}
\hline \multicolumn{4}{c|}{ Primary Contacts } \\
Contact Person & Subject & Fax\# & Phone & E-Mail Address \\
Bill Kator & Inventory & 4240 & 6500 & KATORW@ANLAPS \\
\hline
\end{tabular}

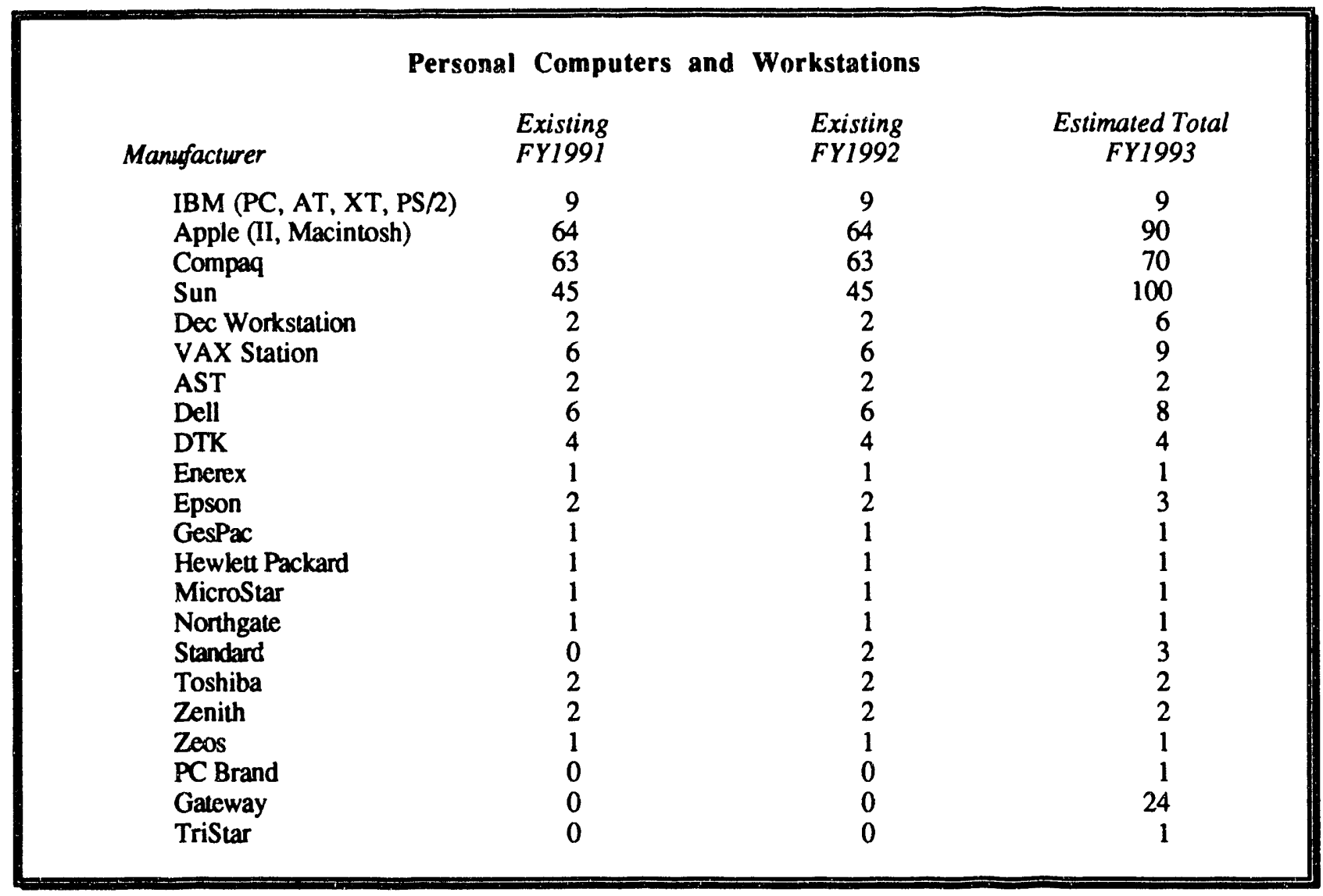




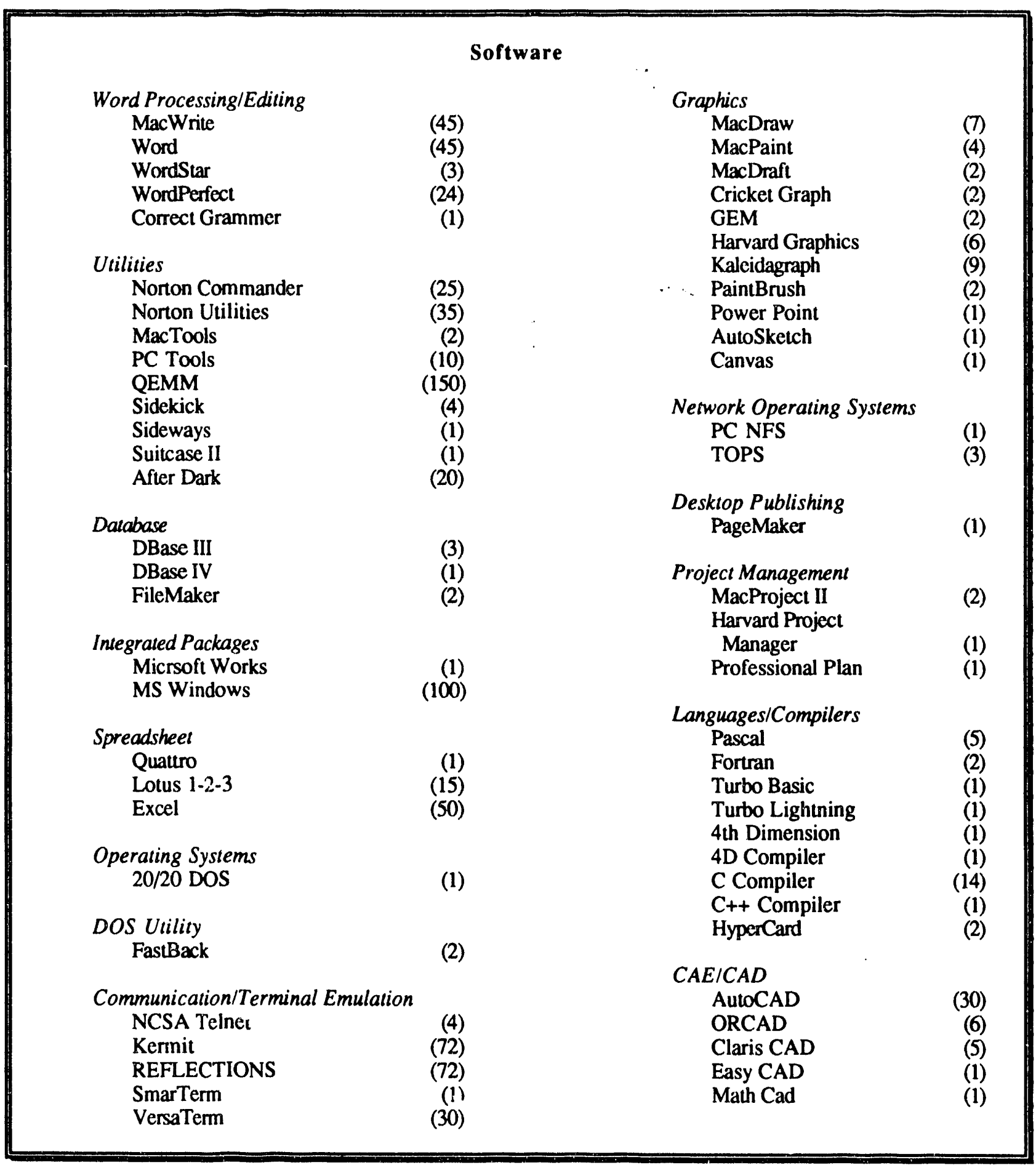




\section{Software (continued)}

\section{Other}

$\begin{array}{lll}\text { Abecos D } & (1) & \text { Back \& Forth } \\ \text { CALA } & (1) & \text { Charisma } \\ \text { Dyno } & (1) & \text { Electrc 5 } \\ \text { Fault } & (1) & \text { GenSize } \\ \text { Image Grabber } & (1) & \text { LabView } \\ \text { Mathematica } & (1) & \text { Mathtype } \\ \text { OpenWindow } & (1) & \text { PE2D } \\ \text { Prototyper } & (1) & \text { Pspice } \\ \text { Square D } & (1) & \text { Symontec } \\ \text { Tosca } & (2) & \text { Trace Calc }\end{array}$

\section{Network Connections}

$\begin{array}{cc}\text { Existing } & \text { Estimated Total } \\ \text { FY1992 } & \text { FY1993 }\end{array}$

Lab-wide Ethernet $\begin{array}{lll}\text { TCP/IP } & 312 & 400\end{array}$

$\begin{array}{lll}\text { DECnet } & 160 & 175\end{array}$

$\begin{array}{lll}\text { Both } & 32 & 50\end{array}$

$\begin{array}{lll}\text { Local AppleTalk } & 60 & 60\end{array}$

Please describe systems and/or applications that you plan to implement during the next fiscal year in the areas of (1) scientific work stations, (2) personal computing, (3) local area networking, and (4) office automation. Please also indicate standardized software packages for your division.

1. Scientific Work Stations: Continue to replace character cell terminals with UNIX and VMS workstations as part of the migration towards distributed computing.

2. Personal Computing: Will still be acquiring PCs due to lower cost software. Will be incorporating them into the distributed computing environment.

3. Local Area Networking: All PCs and workstations are being networked. Two CISCO routers have been installed. The network is being split into two (1) controls and (2) general users with FDDI interconnect.

4. Office Automation: On-line documentation systems for manuals and access to all the CAD drawings are being tested for eventual implementation.

5. Standardized Software: Looking more into data exchange between software packages rather than standardized software. 
Argonne East--Experiment Analysis (Cost Center 104)

\begin{tabular}{|ccccc|}
\hline \multicolumn{4}{c|}{ Primary Contacts } \\
Contact Person & Subject & Fax\# & Phone & E-Mail Address \\
Gary Batte & All & 7340 & 7026 & N/A \\
\hline
\end{tabular}

Personal Computers and Workstations

$\begin{array}{cccc} & \text { Existing } & \text { Existing } & \text { Estimated Total } \\ \text { Manufacturer } & \text { FY1991* } & \text { FY1992 } & \text { FY1993 }\end{array}$

Apple (Mac-Plus, II, ci, cx, si) IBM (PC, AT, XT, All Clones)

Sun Sparc Stations, SLC

Sun Sparc Stations, IPX

NCD X Terminais $15 S$

$\begin{array}{ll}1 & 1 \\ 8 & 8 \\ 2 & 2 \\ 1 & 1 \\ 3 & 3\end{array}$

* No figures available for FY 1991

\section{Software}

Word Processing/Editing WordPerfect (UNIX)

Operating Systems

Windows

DOS
(6)
Spreadsheet

MS Excel

(3)

Networking

DATAVIEWS (UNIX)

1
8
2
1
3


Please describe systems and/or applications that you plan to implement during the next fiscal year in the areas of (1) scientific work stations, (2) personal computing, (3) local area networking, and (4) office automation. Please also indicate standardized software packages for your division.

1. Scientific Work Stations: None planned.

2. Personal Computing: We will increase both in the quantity and use of personal computers. We will look at using one for the tracking of tasks and resources. We have increased the amount and size of databases and are making them available to all personnel.

3. Local Area Networking: We will increase the number of users once we have upgraded to the current version of Novell and will look at bridging to other LANs.

4. Office Automation: CDOCS (Classified Document Control System) is being planned for use by all division personnel.

5. Standardized Software: Word processing, database, spreadsheet, and graphics packages are being evaluated for standardized use by section personnel. 


\title{
Argonne West--Analytical Laboratory (Cost Center 104)
}

\begin{tabular}{|ccccc|}
\hline \multicolumn{4}{c|}{ Primary Contacts } \\
Contact Person & Subject & Fax\# & Phone & E-Mail Address \\
Gary Batte & All & 7340 & 7026 & N/A \\
\hline
\end{tabular}

\section{Personal Computers and Workstations}

$\begin{array}{lllc} & \text { Existing } & \text { Existing } & \text { Estimated Total } \\ \text { Manufacturer } & \text { FY1991* } & \text { FY1992 } & \text { FY1993 }\end{array}$

\begin{abstract}
IBM (PC, AT, XT)
PC Clone (486-xx)

PC Clone (386-xx)

PC Clone (286-xx)
\end{abstract}

Apple (Mac-Plus, II, ci,cx,si)

$\begin{array}{ll}4 & 4 \\ 4 & 4 \\ 1 & 1 \\ 6 & 6 \\ 6 & 6\end{array}$

Figures not available for FY1991

\section{Software}

Word Processing/Editing

MS Word

WordPerfect

Word for Word

Teach Text

Word 1.0.3

Database

DBase IV

DBase III+

Quattro Pro

FoxPro

Operating Systems

Windows

DOS
(1)

(1)

(2)

(1)

(6)

(1)

(4)

(8)
Graphics/CAD

DrawPerfect

Adobe Illustrator

Auto Sketch

Cricket Graph

MacDraw

(1)

(1)

(1)

(1)

(1)

Spreadsheet

Lotus 1-2-3

(1)

MS Excel

(2)

Communication/Terminal Emulation

Tellx

Versaterm Pro 


\section{Software (continued)}

Utility

Norton Utilities

(4)

Virus

HyperCard

Fast Back

QMM

Norton Backup

Languages/Compilers

H.T. Basic

(3)

(1)

(1)
Other

DP500AA

Calendar

MacProject

(1)

Networking

MacLink Translators

(1)

\section{Network Connections}

Existing

FY1992

Novell Netware 386

Lab-Wide Tellabs

DecNet

TCP/IP
0

0

0
Estimated Total FY1993

5

10

10

Please describe systems andlor applications that you plan to implement during the next fiscal year in the areas of (1) scientific work stations, (2) personal computing, (3) local area networking, and (4) office automation. Please also indicate standardized software packages for your division.

1. Scientific Work Stations: None planned.

2. Personal Computing: We will increase both in the quantity and use of personal computers. We will look at using one for the tracking of tasks and resources. We have increased the amount and size of databases and are making them available to all persionnel.

3. Local Area Networking: We will increase the number of users once we have upgraded to ine current version of Novell and will look at bridging to other LANs.

4. Office Automation: CDOCS (Classified Document Control System) is being planned for use by all division personnel.

5. Standardized Software: Word processing, database, spreadsheet, and graphics packages are being evaluated for standardized use by section personnel. 


\section{Argonne West--Bldg T-2/T2A/FMF (Cost Center 104)}

\section{Primary Contacts}

$\begin{array}{ccccc}\text { Contact Person } & \text { Subject } & \text { Fax\# } & \text { Phone } & \text { E-Mail Address } \\ \text { Gary Batte } & \text { All } & 7340 & 7026 & \text { N/A }\end{array}$

\section{Personal Computers and Workstations}

\begin{tabular}{|c|c|c|}
\hline $\begin{array}{l}\text { Existing } \\
\text { FY1991* }\end{array}$ & $\begin{array}{l}\text { Existing } \\
\text { FY1992 }\end{array}$ & $\begin{array}{c}\text { Estimated Total } \\
\text { FY199.3 }\end{array}$ \\
\hline $\begin{array}{l}\text { Apple (Mac-Plus, II, ci, cx, si) } \\
\text { IBM (PC, AT, XT, System 2/80-386) } \\
\text { PC Clone (386xx) } \\
\text { PC Clone (286-xx) } \\
\text { Sun Sparc Station IPX }\end{array}$ & $\begin{array}{r}30 \\
6 \\
7 \\
3 \\
1\end{array}$ & $\begin{array}{r}30 \\
6 \\
7 \\
3 \\
1\end{array}$ \\
\hline
\end{tabular}

* Figures not available for FY 1991

\section{Software}

Word Processing/Editing

MS Word

WordPerfect

Word 4.0

Teach Text

Word 5.0

MacWrite

Correct Grammer

Communication/Terminal Emulation

Tops

Reflection 7

MacLink + Transiators

MacLink Plus

VersaTerm

LapLink

Reflection 4

MacLink PC

Database

DBase IV

Quattro Pro

FoxPro
(3)

(2)

(1)

(2)

(1)

(9)

(1)

(1)

(4)

(4)

(1)

(1)

(1)

(3)

(2)

(1)
Graphics/CAD

DrawPerfect

MacDraft

Auto Sketch

Cricket Graph

MacDraw II

StatView II

File Vision

30 Charts

Generic CADD

Paste Ease

LabView II

MS Chart

Math CAD

Kaleido Graph

MacDraft Graphics

Delta Graph

(1)

(8)

(2)

(1)

(6)

(3)

(2)

(1)

(2)

(3)

(2)

(1)

(1)

(1)

(1)

(2)

Spreadsheet

Lotus 1-2-3

MS Excel

Cricket

Stiffit 


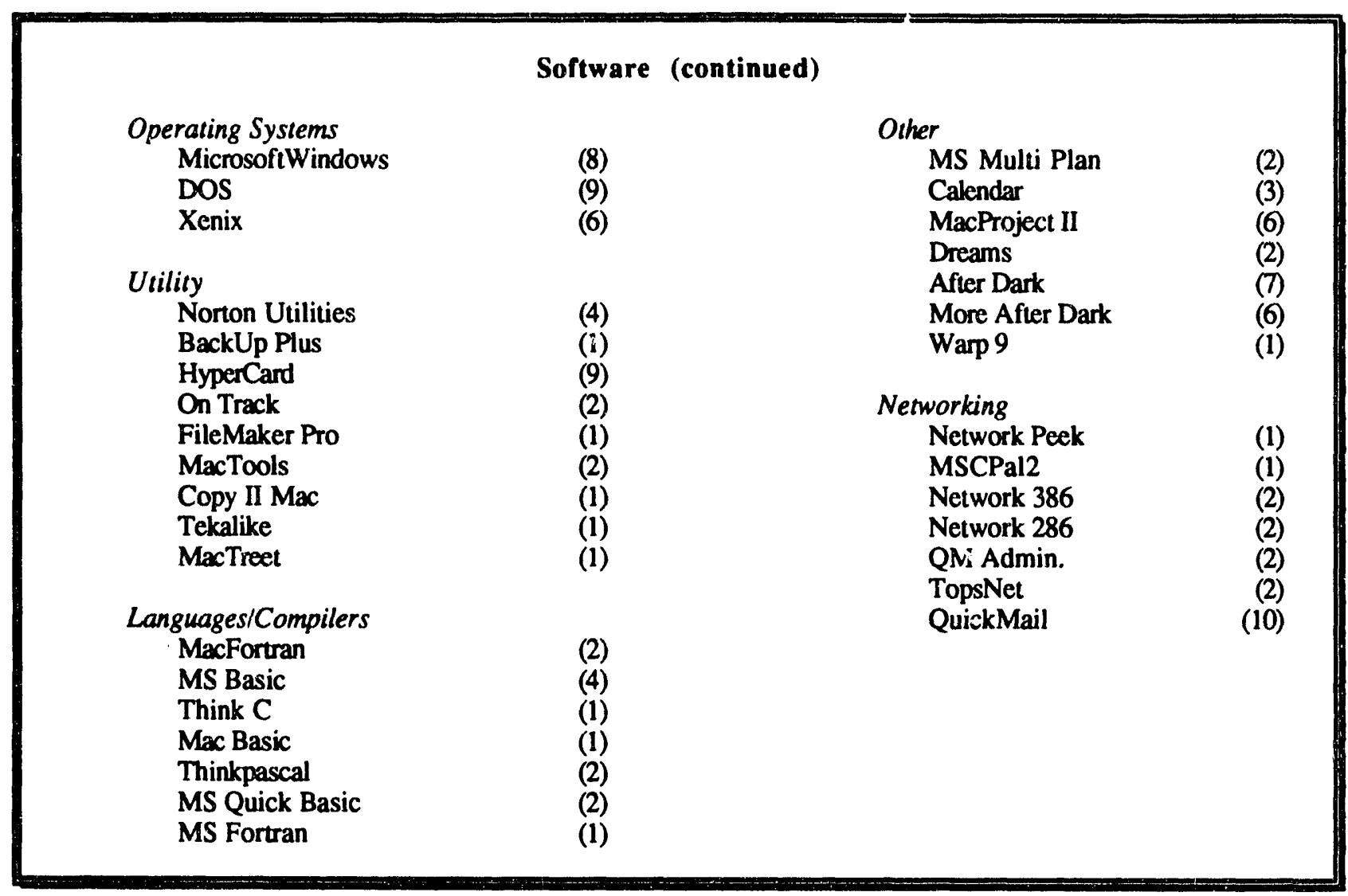

\section{Network Connections}

$\begin{array}{cc}\text { Existing } & \text { Estimated Total } \\ \text { FY1992 } & \text { FY1993 }\end{array}$

Local AppleTalk

Novell Netware 386

14

Lab-Wide Tellabs

10

TCP/IP

14

10

14 
Please describe systems andlor applications that you plan to implement during the next fiscal year in the areas of (1) scientific work stations, (2) personal computing. (3) local area networking, and (4) office automation. Please also indicate standardized software packages for your division.

1. Scientific Work Stations: None planned.

2. Personal Computing: We will increase both in the quantity and use of personal computers. We will look at using one for the tracking of tasks and resources. We have increased the amouni and size of databases and are making them available to all personnel.

3. Local Area Networking: We will increase the number of users once we have upgraded to the current version of Novell and will look at bridging to other LANs.

4. Office Automation: CDOCS (Classified Document Control System) is being planned for use by all division personnel.

5. Standardized Software: Word processing, database, spreadsheet, and graphics packages are being evaluated for standardized use by section personnel. 


\section{Argonne West--Communications and Computing Services (Cost Center 552)}

\begin{tabular}{|ccccc|}
\hline & \multicolumn{5}{c|}{ Primary Contacts } \\
Contact Person & Subject & Fax\# & Phone & E-Mail Address \\
Gary Batte & All & 7340 & 7026 & N/A \\
\hline
\end{tabular}

\section{Personal Computers and Workstations}

$\begin{array}{ccc}\text { Existing } & \text { Existing } & \text { Estimated Total } \\ \text { FY1991 } & \text { FY1992 } & \text { FY1993 }\end{array}$

Apple Mac ci

IBM (PC,AT, XT)

PC Clone (486-xx)

PC Clone (386-xx)

PC Clone (286-xx)

$\begin{array}{lll}0 & 1 & 1 \\ 0 & 6 & 6 \\ 0 & 4 & 4 \\ 0 & 7 & 7 \\ 0 & 5 & 5\end{array}$

\section{Word Processors}

Manufacturer

Existing

FY1991

Existing

FY1992

Estimated Total

FY 1993

SilverReed

1

1

1

\section{Software}

Word Processing/Editing MS Word

(1)

WordPerfect Office

WordPerfect 5.1

WordPerfect Library

Spreadsheet

Lotus 1-2-3

Quattro Pro

Excel
(1)

(11)

(1)

(1)

(4)

(3)
Database

DBase IV

(2)

DBase IIIt+

FoxPro

(4)

Languages/Compilers

MS-Assembler

(1)

MS-C

MS-Basic

Quick-C

Lattice-C
(1)

(2)

(1)

(1) 


\section{Software (continued)}

Networking and Utilities

Novell Netware 386

V3.11 (100 users)

Novell Netware 286 V2.15

LAN Watch

CC: Mail

EtherPeek

ORPS

TrackNet 2000

DataSecurity Plus

Pathworks/PC

Gateway

PC/TCP

\section{Communication/Terminal Emulation}

Reflection $2 / 4 / 7$

Rlink7

Kermit

(2)

Versaterm Pro

CrossTalk

Spreadsheet

Lotus 1-2-3

Quatro Pro

Excel

Operating Systems

$\mathrm{OS} / 2$

Microsoft Windows

Dos
(1)

(4)

(5)

(1)

(7)
Utility

QEMM

Soft PC

(2)

(1)

Virus Scan

Norton Utilities

Hyper Card

(4)

(1)

Norton Backup

Languages/Compilers

MS-Assembler

MS-C

MS-Basic

Quick-C

Lattice-C

Word Processing/Editing

MS Word

WordPerfect Office

WordPerfect 5.1

Word Perfect Library

CAE/CAD Graphics

Designer

Flowchart III

Auto Sketch

Auto CAD

\section{Network Connections}

\section{Existing \\ FY1992}

Novell Netware 386

Lab-Wide Te:llabs

DecNet

TCP/IP
40

6

1

\section{Estimated Total} FY1993 
Please describe systems and/or applications that you plan to implement during the next fiscal year in the areas of (1) scientific work stations, (2) personal computing, (3) local area networking, and (4) office automation. Please also indicate standardized software packages for your division.

1. Scientific Work Stations: None planned.

2. Personal Computing: We will increase both in the quantity and use of personal computers. We will look at using one for the tracking of tasks and resources. We have increased the amount and size of databases and are making them available to all personnel.

3. Local Area Networking: We will increase the number of users once we have upgraded to the current version of Novell and will look at bridging to other LANs. There are currently 5 Novell LANs on site that are connected together.

4. Office Automation: CDOCS (Classified Document Control System) is being planned for use by all division personnel.

5. Standardized Software: Word processing, database, spreadsheet and graphics applications will be evaluated for standard implementation by all section personnel to provide consistency and ease of transfer. 


\section{Argonne West-. Fuel Cycle (Cost Center 118)}

Primary Contacts

Contact Person

Subject

Fax\#

Phone

E-Mail Address

C.L.Beck

All

7151

7463

N/A

\section{Personal Computers and Workstations}

Manufacturer

SUN SPARC

Silicon Graphics

IBM \& Clones (86)

(286)

(386)

(486)

(NoteBook)

Apple MACs (512)

(Plus)

(II)

(NoteBook)

\section{Existing \\ FY1991}

2
0
12
20
35
2
0
7
6
30
2

\section{Existing}

FY1992
Estimated Total FY1993

\section{Software*}

Word Processing/Editing

WordPerfect 5.0

Word

Spreadsheet

Lotus 1-2-3

Quattro Pro

Communication/Terminal Emulation

Kermit

Reflections
Database

DBase IV

Fox Pro

CAD/Graphics

AutoCAD

Project Mangement

Primivera
2

1

12

20

55

12

2

7

6

40

4

* Numbers of software packages not available. 


\section{Network Connections}

Existing
FY1992
Estimated Total
FY1993

Please describe systems andlor applications that you plan to implement during the next fiscal year in the areas of (I) scientific work stations, (2) personal computing. (3) local area networking, and (4) office automation. Please also indicate standardized software packages for your division.

1. Scientific Work Stations: None planned.

2. Personal Computing: Continued purchase of PC's and MACs for office automation workstations as required.

3. Local Area Networking: Novell--a 100-user license will be purchased for integrating all PC and MAC users into a Division-wide local area network for administrative, office automation and communications convenience. Installations of 10BaseT (twisted) pair hubs connected to an ANL-W ethemet backbone for each building and premise distribution system for in-house wiring to all divisional offices requiring network connectivity.

4. Office Automation: WordPerfect office will be purchased for scheduling and general information management for individuals in FCD. Purchase a divisional server with centralized file backup/archival capabilities and file server resources to optimize software and data base management. E-Mail capability will be developed for PCs and MACs.

5. Standardized Software:

WordPerfect 5.0

Primivera

AutoCad

WordPerfect Office

cc:Mail

Quick Mail 


\section{Argonne West--Machine Shop (Cost Center 554)}

\begin{tabular}{|ccccc|}
\hline \multicolumn{5}{c|}{ Primary Contacts } \\
Contact Person & Subject & Fax\# & Phone & E-Mail Address \\
Larry Spencer & All & 7738 & 7411 & N/A \\
\hline
\end{tabular}

\begin{tabular}{|cccc|}
\hline & Personal Computers & and Workstations \\
& Existing & Existing & Estimated Total \\
FYI993 & FY1992 & \\
Manufacturer & FY1991 & & 1 \\
MIS 386SX-16 & 0 & 1 & 1 \\
ACT & 0 & 1 & 1 \\
HP Laser Jet IIP & 0 & 1 & \\
\hline
\end{tabular}

\section{Word Processors}

Manufacturer

Xerox Model 6040

\section{Existing}

FY1991

1
Existing

FY1992

1
Estimated Total FY1993

\section{Software}

Word Processing/Editing

WordPerfect

(1)

Communication/Terminal Emulation

Other

Reflections

(1)

1 
Please describe systems and/or applications that you plan to implement during the next fiscal year in the areas of (1) scientific work stations, (2) personal computing, (3) local area networking, and (4) office automation. Please also indicate standardized software packages for your division.

1. Scientific Work Stations: None planned.

2. Personal Computing: Software upgrade and improved peripheral equipment.

3. Local Area Networking: None planned.

4. Office Automation: Continue to develop and implement office automation through improvements in software and computer capabilities.

5. Standardized Software: WordPerfect, Kermit, Reflections. 


\section{Argonne West--Office of Operational Safety (Cost Center 570)}

\begin{tabular}{|rcccc|}
\hline \multicolumn{4}{c|}{ Primary Contacts } \\
Contact Person & Subject & Fax\# & Phone & E-Mail Address \\
Lori DeLuca & All & 7655 & 7109 & N/A \\
\hline
\end{tabular}

Personal Computers and Workstations

$\begin{array}{cccc} & \text { Existing } & \text { Existing } & \text { Estimated Total } \\ \text { Manufacturer } & \text { FY1991* } & \text { FY1992 } & \text { FY1993 }\end{array}$

Gateway 2000

USAPC

Maclisi

Macintosh IIci, Powerbook 170

MiS

$\begin{array}{ll}1 & 1 \\ 4 & 8 \\ 1 & 1 \\ 1 & 1 \\ 1 & 1\end{array}$

* Figures for FY1991 are unavailable.

\begin{tabular}{|c|c|c|c|}
\hline \multicolumn{4}{|c|}{ Software } \\
\hline Word Processing & & Spreadsheet & \\
\hline WordPerfect (IBM) & (5) & Microsoft Chart & (1) \\
\hline QueSoftware's Right Writer & (1) & Microsoft Excel & (1) \\
\hline Word 4.0 & (1) & Microsoft Multiplan & (1) \\
\hline Word 5.0 & (2) & Lotus 1-2-3 & (3) \\
\hline WordPerfect $2.0(\mathrm{Mac})$ & (1) & & \\
\hline & & Project Management & \\
\hline Utilities & & MacProject & (1) \\
\hline Marstek's Hand Scanner & & & \\
\hline Utility Program & (1) & Desktop Publishing & \\
\hline MacTools Deluxe & (1) & Pagemaker 4.0 & (1) \\
\hline Communication/Terminal Emulation & & Languages/Compilers & \\
\hline VersaTerm Pro & (1) & MacFortran/020 & (1) \\
\hline & & Microsoft Basic & (1) \\
\hline Graphics & & Microsoft Fortran & (1) \\
\hline Z-Soft's PaintBrush for DAK & (1) & & \\
\hline MacDraw & (2) & $C A E / C A D$ & \\
\hline SuperPaint & (1) & MacDraft & (1) \\
\hline CricketDraw & (1) & MathCAD & (1) \\
\hline PowerPoint & (1) & & \\
\hline
\end{tabular}




\section{Software (continued)}

Other

Marstek's Perceive

Personal Omnifont

OCR

YGA $1024 / \mathrm{D} / \mathrm{XL} / \mathrm{DXL}$

Version 3.1

First Things First

StatView 512+

Microsoft MS-DOS

Version 5.0
Alpha Four

Reason

Microsoft Mouse

Setup

Filevision

MacTerminal

Panorama 1.5
(1) AMI-PRO

(1) MS-DOS 4.01

Microsoft Windows 3.1

(1) Copy II Mac/Mac Tools

(1) Filevision IV

(1) Mindset

(1) Telalike TK! Solver
(1)

(1)

(1)

(1)

(1)

(1)

(1)

\section{Network Connections}

$\begin{array}{cc}\text { Existing } & \text { Estimated Total } \\ \text { FY1992 } & \text { FY1993 }\end{array}$

Novell

0

12

Please describe systems and/or applications that you plan to implement during the next fiscal year in the areas of $(1)$ scientific work stations, (2) personal computing, (3) local area networking, and (4) office automation. Please also indicate standardized software packages for your division.

1. Scientific Work Stations: None planned.

2. Personal Computing: Increase software as the job requires it.

3. Local Area Networking: All PCs should have Ethemet.

4. Office Automation: None planned.

5. Standardized Software: None indicated. 


\section{Argonne West--Office of Quality Assurance (Cost Center 102)}

\begin{tabular}{|lcccc|}
\hline \multicolumn{4}{c|}{ Primary Contacts } \\
Contact Person & Subject & Fax\# & Phone & E-Mail Address \\
Ellen Christensen & All & 7488 & 7523 & N/A \\
\hline
\end{tabular}

\begin{tabular}{|lccc|}
\hline \multicolumn{2}{c|}{ Personal Computers and Workstations } \\
& Existing & Existing & Estimated Total \\
& FY1991 & FY1992 & \\
Manufacturer & & & 2 \\
IBM (PC, AT, XT, PS 2$)$ & 1 & 3 & 1 \\
USA & 0 & 0 & 3 \\
Macintosh & 0 & 0 & 1 \\
Bentley & 0 & 0 & 1 \\
Club American & 0 & 0 & \\
\hline
\end{tabular}

\begin{tabular}{|llll|}
\hline & Software & & \\
Database & & & \\
DBase III Plus & $(1)$ & Word Processing & WordPerfoct \\
DBase II & $(1)$ & Microsoft Word & $(3)$ \\
Other & & Mass-11 & $(1)$ \\
Paradox & $(1)$ & Utilities & \\
DOS & $(5)$ & Norton Utilities & $(1)$ \\
Filemaker Pro & $(1)$ & Sidekick & $(1)$ \\
Fastback & $(1)$ & Graphics & \\
ORPS & $(2)$ & MacDraft & Super Paint \\
ABC Flowcharter & $(1)$ & & $(1)$ \\
Windows & $(2)$ & Spreadsheet & Excel \\
Quick Mail & $(2)$ & Quautro & $(3)$ \\
Writing Assistant & $(1)$ & Quattro Pro & $(1)$ \\
Communication/Terminal Emulation & & Lotus 1-2-3 & $(1)$ \\
Kermit & $(1)$ & & $(1)$ \\
Reflections & $(2)$ & & \\
Project Management & & & \\
Mac Project & $(1)$ & & \\
\hline
\end{tabular}


Please describe systems and/or applicalions that you plan to implement during the next fiscal year in the areas of (I) scientific work stations, (2) personal computing. (3) local area networking, and (4) office automation. Please also indicate standardized sof tware packages for your division.

1. Scientific Work Stations: None planned.

2. Personal Computing: None planned.

3. Local Area Networking: None planned.

4. Office Automation: None planned.

5. Standardized Software: None indicated. 


\section{Argonne West--Plant Services (Cost Center 557)}

\section{Primary Contacts}

Contact Person

Subject

Fax\#

Phone

E-Mail Address

Everet A. Clifton

All

7030

7680

N/A

\section{Personal Computers and Workstations}

$\begin{array}{cccc} & \text { Existing } & \text { Existing } & \text { Estimated Total } \\ \text { Manufacture } & \text { FY1991 } & \text { FY1992 } & \text { FY1993 }\end{array}$

Club American

APS

MIS 386SX-16

Gateway 286

IBM PS-2

IBM 8086

$\begin{array}{ll}5 & 4 \\ 1 & 0 \\ 8 & 8 \\ 1 & 1 \\ 1 & 1 \\ 0 & 1\end{array}$

\section{Word Processors}

Manufacturer

IBM Wheelwriter 6

Xerox 620

Xerox 6020

IBM Wheeleriter 30

\section{Existing}

FY1991

2
1
1
1

Existing

FY1992

Estimated Total FY 1993

\section{Software}

Word Processing/Editing

Mass-11

(4)

Deskmate

WordPerfect 5.1

Database

DBase III Plus

DBase IV

Spreadsheet

Lotus 1-2-3

Quattro Pro
(6)

(1)
Graphics

AutoSketch
DrawPerfect

(5)

(2)

Desktop Publishing

Always

Sideways

(3)

(1)

Security

FluShol

PC-Vault
2 
Software (continued)

Other
Registrar
Regscan
TopPriority
Ladder
MicroSoft Project
IBM Info Window
Calendar Creator
Fast Regs
SARA
Form Tools
Whereabouts
Windows
Plan Perfect
Ami Pro

(1)

(2)

(4)

(1)

\section{Utilities}

Norton Commander

Wonder

Mouse

Norton Utilities

Rapid File

(11)

Communication/Terminal Emulation

Kermit

(8)

Project Management

Project Management
(2)

Please describe systems and/or applications that you plan to implement during the next fiscal year in the areas of (1) scientific work stations, (2) personal computing, (3) local area networking, and (4) office automation. Please also indicate standardized software packages for your division.

1. Scientific Work Stations: None planned.

2. Personal Computing: 3 PCs capable of handling the Graphics that is required by IFR Maintenance.

3. Local Area Networking: LAN System for the WCG with capabilities to handle at least 10 connections.

4. Office Automation: None planned.

5. Standardized Software: OPmist for scheduling; Fox Pro's Database to replace pundit. 
Argonne West Reactor Program Services Division--Accounting (Cost Center 533)

\begin{tabular}{|ccccc|}
\hline \multicolumn{4}{c|}{ Primary Contacts } \\
Contact Person & Subject & Fax\# & Phone & E-Mail Address \\
Susan Sommers & All & N/A & 7245 & N/A \\
\hline
\end{tabular}

\section{Personal Computers and Workstations}

$\begin{array}{cccc}\text { Manufacturer } & \begin{array}{c}\text { Existing } \\ \text { FY1991 }\end{array} & \begin{array}{c}\text { Existing } \\ \text { FY1992 }\end{array} & \begin{array}{c}\text { Estimated Total } \\ \text { FY1993 }\end{array} \\ \text { MIS } & 1 & & 1 \\ \text { PC-USA } & 0 & 1 & 2\end{array}$

Software

Word Processing/Editing WordPerfect

Spreadsheet

Lotus 1-2-3

(1)

Communication/Terminal Emulation

Reflections

(3)

Preface

(1)

\section{Network Connections \\ Existing \\ FY1992 \\ Estimated Total FY1993}

Lab-wide Ethemet TCP/IP
2

3 
Planned activities and/or applications to be implemented during the next fiscal year in the areas of (1) scientific work stations, (2) personal computing. (3) local area networking, and (4) office automation. Please also indicate standardized software packages for you division.

1. Scientific Work Stations: None planned.

2. Persomal Computing: None planned.

3. Local Area Networking: Lab-wide Ethernet TCP/IP (1 planned).

4. Office Automation: None planned.

5. Standardiaed Software: None indicated. 
Argonne West Reactor Program Services Division--Division Office (Cost Center 530)

\begin{tabular}{|ccccc|}
\hline \multicolumn{5}{c|}{ Primary Contacts } \\
Contact Person & Subject & Fax\# & Phone & E-Mail Address \\
Jerry Ward & All & 7655 & 7108 & N/A \\
\hline
\end{tabular}

\begin{tabular}{|cccc|}
\hline & $\begin{array}{c}\text { Personal Computers } \\
\text { and }\end{array}$ & & \\
Manufacturer & Existing & Existing & Estimated Total \\
& FY1991 & FY1992 & \\
PC Limited & 1 & 0 & 0 \\
ACT 386 & 3 & 2 & 2 \\
ALTECH & 2 & 0 & 0 \\
MacIntosh & 4 & 1 & 1 \\
MIS & 2 & 1 & 1 \\
Gateway 2000 & 1 & 2 & 2 \\
\hline
\end{tabular}

\section{Word Processors}

Manufacturer

Existing

FY1991

Existing

FY1992

Estimated Total FY 1993

$\begin{array}{llll}\text { NBI } & 0 & 0 & 0 \\ \text { Xerox } & 1 & 0 & 0\end{array}$

\section{Softrvare}

Word Processing/Editing

Word

WordPerfect

(1)

Graphics

MacDraft

PC-Plot

Auto Sketch

Freelance

Communication/Terminal Emulation

Kermit

Reflections

Ethernet

(1)

(4)

(2)
Utility

Windows 3.0

(4)

Norton Backup

Excel

(1)

(3)

Screenblanker

(3)

PC Guardian

(1)

Norton Commander

Personal Advocate

Lexis

(1)

(1)

(1)

Dauabase

DBase IV

(1) 


\section{Network Connections}

$\begin{array}{lc}\text { Existing } & \text { Estimated Total } \\ \text { FY1992 } & \text { FY1993 }\end{array}$

L.ab-wide Ethemet TCP/IP

Planned activities and/or applications to be implemented during the next fiscal year in the areas of (1) scientific work stations, (2) personal computing, (3) local area networking, and (4) office automation. Please also indicate standardized software packages for you division.

1. Scientific Work Stations: None planned.

2. Personal Computing: None planned.

3. Local Area Networking: Lab-wide Ethernet TCP/IP (1 planned).

4. Office Automation: None planned.

5. Standardiaed Software: None indicated. 


\section{Argonne West Reactor Program Services Division--Environmental Waste Management (Cost Center 536)}

\begin{tabular}{|lcccc|}
\hline \multicolumn{4}{c|}{ Primary Contacts } \\
Contact Person & Subject & Fax\# & Phone & E-Mail Address \\
G. C. Marshall & All & 7344 & 7666 & N/A \\
\hline
\end{tabular}

Personal Computers and Workstations

$\begin{array}{cccc} & \text { Existing } & \text { Existing } & \text { Estimated Total } \\ \text { Manufacturer } & \text { FY1991 } & \text { FY1992 } & \text { FY1993 }\end{array}$

$\begin{array}{lrrr}\text { PC-USA } & 1 & 13 & 18 \\ \text { ACT } & 3 & 3 & 3 \\ \text { Galeway } & 1 & 1 & 1 \\ \text { DTK } & 1 & 1 & 1 \\ \text { ZIP } & 2 & 2 & 2 \\ \text { Macintosh } & 1 & 1 & 1\end{array}$

Software

Word Processing/Editing

WordPerfect

(20)

Utility

Windows

PC Tools

(18)

Norton Utilities

(6)

Database

DBase

(9)

FoxPro

Quattro Pro

FileMaker Pro

Communications/Terminal Emulation

Kermit

(12)

RLink 3

Pro Com

CCPlus

Graphics

Harvard Graphics

DrawPerfect

MicroGraphics Draw

Auto Sketch

Other

RegScan

Comply

Alpha IV

Sidekick

OrgChart

OHS

(2)

(i)

\section{Spreadsheet}

Lotus 1-2-3

Excel 


\section{Network Connections}

Existing

FY1992
Estimated Total

FY1993

Lab-wide Tellabs

7

14

Planned activities and/or applications to be implemented during the next fiscal year in the areas of (1) scientific work stations, (2) personal computing. (3) local area networking, and (4) office automation. Please also indicate standardized software packages for you division.

1. Scientific Work Stations: None planned.

2. Personal Computing: Upgrading of all PCs and software (PC Tools, FoxPro, DrawPerfect, etc.) will continue in FY1993. This will allow increased use of PCs for general applications and creating/maintaining information tracking databases.

3. Local Area Networking: None planned.

4. Office Automation: None planned.

5. Standardiaed Software: None indicated. 
Argonne West Reactor Program Services Division--Food Services (Cost Center 559)

\begin{tabular}{|rcccc|}
\hline \multicolumn{5}{c|}{ Primary Contacts } \\
Contact Person & Subject & Fax\# & Phone & E-Mail Address \\
Dave Krekel & All & 7738 & 7108 & N/A \\
\hline
\end{tabular}

Cost Center 559 has no hardware, software or network connections 
Argonne West Reactor Program Services Division--Human Resources (Cost Center 531)

\begin{tabular}{|ccccc|}
\hline \multicolumn{4}{c|}{ Primary Contacts } \\
Contact Person & Subject & Fax\# & Phone & E-Mail Address \\
Shelley Wray & All & 7366 & 7323 & N/A \\
\hline
\end{tabular}

\begin{tabular}{|cccc|}
\hline \multicolumn{3}{c|}{ Personal Computers } & and Workstations \\
& Existing & Existing & Estimated Total \\
Manufacturer & FY1991 & FY1992 & FY1993 \\
IBM (PC, AT, XT, PS/2) & 1 & 1 & 1 \\
Club American & 1 & 1 & 2 \\
Altec & 0 & 0 & 4 \\
MIS & 0 & 0 & 2 \\
PC-USA & 0 & 0 & 1 \\
\hline
\end{tabular}

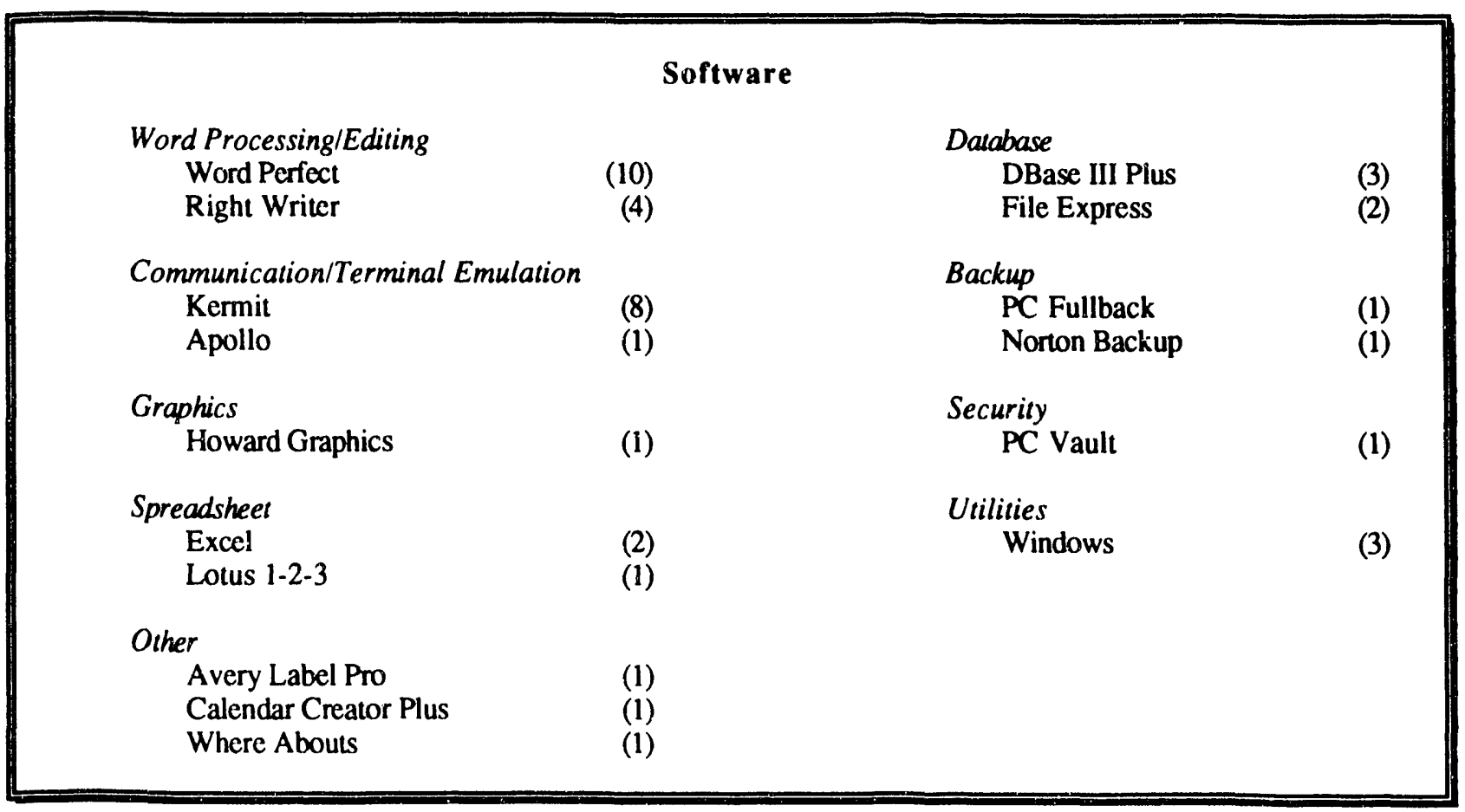


Please describe systems andlor applications that you plan to implement during the next fiscal year in the areas of $(1)$ scientific work stations, (2) personal computing, (3) local area networking, and (4) office automation. Please also indicate standardized software packages for your division.

1. Scientific Work Stations: None planned.

2. Personal Computing: None planned.

3. Local Area Networking: None planned.

4. Office Automation: None planned.

5. Standardized Software: None indicated. 
Argonne West Reactor Program Services Division--Information Services (Cost Center 537)

\begin{tabular}{|ccccc|}
\hline \multicolumn{3}{c|}{ Primary Contacts } \\
Contact Person & Subject & Fax\# & Phone & E-Mail Address \\
& & & & N/A \\
John K. Ammon & All & 7656 & 7507 & N/A \\
Judy Krieger & All & 7656 & 7237 & \\
& & & \\
\hline
\end{tabular}

\begin{tabular}{|lccc|}
\hline & & & \\
& & & \\
& & & \\
& Existing & Existing & Estimated Total \\
Manufacturer & FYl991 & FY1993 & 1 \\
IBM (PC, AT, XT, PS/2) & 4 & 5 & 2 \\
ACT (386-25) & 2 & 2 & 1 \\
Altcc (386-25) & 1 & 1 & 2 \\
PC-USA & 1 & 1 & 2 \\
\hline
\end{tabular}

\section{Software}

Utility

FastBack Plus

Norton Utilities

OCLC

Communication/Terminal Emulation

Kermit

Telix
(4)

(4)

(3)

(1)
Word Processing/Editing WordPerfect

(4)

Imaging Software

Wilson Disk

Current Contents

ASME Vessel Code

Computer Library

Silver Platter

Science Citation
(2)

(2)

(2)

(2)

(2)

(2)

\section{Network Connections}

Existing

FY1992 Estimated Total
FY1993 
Please describe systems and/or applications that you plan to implement during the next fiscal year in the areas of (1) scientific work stations, (2) personal computing, (3) local area networking, and (4) office automation. Please also indicate standardized software packages for your division.

1. Scientific Work Stations: None planned.

2. Personal Computing: We will increase both in the quantity and use of personal computers. We have increased the amount and size of databases and are making them available to all personnel.

3. Local Area Networking: None planned.

4. Office Automation: We have implemented ASKSAM as book, journal, and periodicals tracking system. This is incorporated with a bar code scanning system.

5. Standardized Software: We have adopted DBase as our database software and WordPerfect as our word processing software. 


\section{Argonne West Reactor Program Services Division-.-Procurement (Cost Center 534)}

\section{Primary Contacts}

$\begin{array}{ccccc}\text { Contact Person } & \text { Subject } & \text { Fax } & \text { Phone } & \text { E-Mail Address } \\ \text { G.B. Wray } & \text { All } & 7283 & 7243 & \text { N/A }\end{array}$

Personal Computers and Workstations

$\begin{array}{cccc} & \text { Existing } & \text { Existing } & \text { Estimated Total } \\ \text { Manufacturer } & \text { FY1991 } & \text { FY1992 } & \text { FY } 1993\end{array}$

$\begin{array}{llll}\text { Galeway } & 4 & 1 & 1 \\ \text { ACT } & 4 & 4 & 4 \\ \text { ALTEC } & 2 & 2 & 2 \\ \text { PC USA } & 0 & 4 & 4\end{array}$

\section{Software}

Word Processing/Editing

Mass-11

Word Perfoct

Communication/Terminal Emulation

Reflections

\section{Database}

DBase III Plus

Lotus 1-2-3

Quattro Pro

Please describe systems andior applications that you plari to implement during the next fiscal year in the areas of (I) scientific work stations. (2) personal computing, (3) local area networking, and (4) office automation. Please also indicase slandardized sof ware packages for your divisior.

1. Scientific Work Stations: None planned.

2. Personal Computing: None planned.

3. Local Area Networking: Establish procurement LAN w/Novell Netware 286 Version 2.15 .

4. Office Automation: None planned.

5. Standardized Software: None indicated. 


\section{Argonne West Reactor Program Services Division--Radiation Fire Safety (Cost} Center 551)

\begin{tabular}{|ccccc|}
\hline \multicolumn{5}{c|}{ Primary Contacts } \\
Contact Person & Subject & Faxt & Phone & E-Mail Address \\
G. C. Marshall & All & 7344 & 7666 & N/A \\
\hline
\end{tabular}

Personal Computers and Workstations

$\begin{array}{lccc}\text { Manufacturer } & \begin{array}{c}\text { Existing } \\ \text { FY1991 }\end{array} & \begin{array}{c}\text { Existing } \\ \text { FY1992 }\end{array} & \begin{array}{c}\text { Estimated Total } \\ \text { FY1993 }\end{array} \\ \text { PC-USA } & 0 & 2 & 8 \\ \text { ACT } & 5 & 5 & 5 \\ \text { Gateway } & 2 & 2 & 2 \\ \text { MIS } & 1 & 1 & 1 \\ \text { ZIP } & 1 & 1 & 1 \\ \text { CLUB } & 4 & 4 & 4 \\ \text { IBM } & 2 & 2 & 2 \\ \text { Computer Direct } & 1 & 1 & 1\end{array}$

\section{Software}

Word Processing/Editing

WordPerfoct

Spreadsheet

Lotus 1-2-3

Excel

(8)

(3)

Communication/Terminal Emulation

Kermit

RLink

PC Anywhere

Reflectiuns 7
(1)
Utility

Windows

PC Tools

(10)

Norton Commander

(12)

(1)

Graphics

TecGraph Pad

(1)

DrawPerfect

PC Paint

Database

DBase

(6)

Languages/Compilers

Fortran

(2) 


\section{Software (continued)}

Other

Maestro II

Cindy

GENII

MIRDOSE

AirDose

$\begin{array}{ll}\text { (1) } & \text { RSAC4 } \\ \text { (3) } & \text { X-Com } \\ \text { (1) } & \text { QAD-FN } \\ \text { (1) } & \text { COOSA } \\ \text { (3) } & \text { RPTExam }\end{array}$

(3)

RadDocay

HAAS

(14)

(2)

(1) REMEDY

(1)

(1) Varskin

(7) ACS

(13)

\section{Network Connections}

Existing

FY1992

Lab-wide Tellabs
12
Estimated Total FY1993

Planned activities and/or applications to be implemented during the next fiscal year in the areas of (1) scientific, work stations, (2) personal computing, (3) local area networking, and (4) office automation. Please also indicale standardized software packages for you division.

1. Scientific Work Stations: None planned.

2. Personal Computing: Upgrading of all PCs and software will continue in FY1993. This will allow increased use of PCs for general applications and creating/maintaining information tracking databases.

3. Local Area Networking: None planned.

4. Office Automation: None planned.

5. Standardized Software: None indicated. 
Argonne West Reactor Program Services Division--Receiving and Inspection (Cost Center 561)

\begin{tabular}{|ccccc|}
\hline \multicolumn{5}{c|}{ Primary Contacts } \\
Contact Person & Subject & Fax\# & Phone & E-Mail Address \\
Dave Krekel & All & 7738 & 7495 & N/A \\
\hline
\end{tabular}

Personal Computers and Workstations

$\begin{array}{lccc}\text { Manufacturer } & \begin{array}{c}\text { Existing } \\ \text { FYl991 }\end{array} & \begin{array}{c}\text { Existing } \\ \text { FY1992 }\end{array} & \begin{array}{c}\text { Estimated Total } \\ \text { FY1993 }\end{array} \\ \text { IBM (PC, AT, XT, PS/2) } & 1 & & 2 \\ \text { PC Limited } & 2 & 2 & 2 \\ \text { Hewlet Packard } & 1 & 1 & 1 \\ \text { PC-USA } & 0 & 1 & 1\end{array}$

\section{Software}

Word Processing/Editing WordPerfect

Mass-11

Database

DBase III Plus

CALPRO

Desktop Publishing

PageMaker

(4)

(2)

Other

FastBack

Reflections

(1)
Utility

Norton Utilities

(2)

Windows

(1)

Communication/Terminal Emulation

Kermit

(1)

Spreadsheet

Excel 
Planned activities and/or applications to be implemented during the next fiscal year in the areas of (1) scientific work stations, (2) personal computing, (3) local area networking, and (4) office automation. Please also indicate standardized software packages for you division.

1. Scientific Work Stations: None planned.

2. Personal Computing: Implenenting access to ORPS for Occurrence Reporting.

3. Local Area Networking: None planned.

4. Office Automation: Institute tracking and trending of action items.

5. Standardized Software: None indicated. 
Argonne West Reactor Program Services Division--Safeguards (Cost Center 532)

\begin{tabular}{|ccccc|}
\hline \multicolumn{5}{c|}{ Primary Contacts } \\
Contact Person & Subject & Fax\# & Phone & E-Mail Address \\
Manny Sanchez & All & 7915 & 7347 & N/A \\
\hline
\end{tabular}

\section{Personal Computers and Workstations}

$\begin{array}{cccc} & \text { Existing } & \text { Existing } & \text { Estimated Tolal } \\ \text { Manufacturer } & \text { FY1991 } & \text { FY1992 } & \text { FY1993 }\end{array}$

IBM (PC, AT, XT, PS/2 13

MAC (II CX, II, II SI, SE, Plus) 12

PC Limited

Club American

12
2

Gateway 2

ACT

PC-USA

ALTEC

SeeQua

Unknown (on order)

Existing
FY1992

FY1993

Software

Database

DBase II/III

FoxPro

SQL Server

Fox Base

Forest Trees

Erwin

Repon Writer

Languages/Compilers

Object View

Aztec C

Thinlee

Graphics

MacPaint

Dreams

Spreadsheel

Excel
(5)

(1)

(1)

(1)

(1)

(2)

(1)

(1)

(29)
Word Processing/Editing

MacWrite

Gramrnatik IV

Word

WordPerfect

WordStar

Windows

(5)

(9)

(4)

(17)

(4)

(16)

Utilities

Statistics

--Statview II

--JMP

Norton Commander

Norton Utilities

(1)

(1)

(2)

(2)

Communication /Terminal Emulation

Kermit

(7) 
Planned activities and/or applications to be implemented during the next fiscal year in the areas of (I) scientific work stations, (2) personal computing, (3) local area networking, and (4) office automation. Please also indicate standardized software packages for you division.

1. Scientific Work Stations: None planned.

2. Personal Computing: None planned.

3. Local Area Nenworking: A database server will be connected to 10 workstations using Microsoft LAN Manager networking software. File storage, backup, and print spooling services will also be needed.

4. Office Automation: None planned.

5. Standardized Software: None indicated. 


\section{Argonne West Reactor Program Services Division--Security (Cost Center 535)}

\begin{tabular}{|ccccc|}
\hline \multicolumn{5}{c}{ Primary Contacts } \\
Contact Person & Subject & Fax & Phone & E-Mail Address \\
Manny Sanchez & All & 7915 & 7347 & N/A \\
\hline
\end{tabular}

\section{Personal Computers and Workstations}

Manufacturer

IBM (PC, AT, XT, PS/2)

PC Limited

Club American

Hewlet Packard

MIS Computer

ACT

Microcomputer

\section{Existing \\ FY1991}

2
3
2
1
5
0
0

Existing
FY1992

2

3

2

0

6

1
Estimated Total FY1993

\section{Software}

Word Processing/Editing

WordPerfect

Grammatic

Leading Edge

Spreadsheet

Express Calc

Easy Flow

Database

DBase III Plus

File Express

QFiler

\section{Utilities}

MS Windows

Norton Utilities

Tool Box

Everex Board
(9)

(2)

(1)
Graphics

Express Graphics

One Step Graphic \&

Palette Plus

Surfer

Image

Corp Ladder

Formtools Gold

DrawPerfect

(3)

(1)

(1)

(1)

(1)

(1)

(1)

Security

G $6000 \mathrm{Key}$ and Core

Control

Assess VA

(1)

PC Vault

FTR

Superlok 300

JTA

DOE Deadly Force 
Software (continued)

Other

DOS

PC Fullback

Reflections

VGA Wonder

Microsoft Serial Mouse

Lantastics

SL

Auto Convert

Logitech
(2)

(1)
CAE/CAD

US Metric Conversion

MathCAD

Generic CAD

(1)

(1)

(1)

Project Management

Softbytes

TRACK

(1)

(2)

\section{Network Connections}

\section{Existing}

FY1992

Lab-wide Tellab

2 Estimated Total
FY1993

Please describe systems andlor applications that you plan to implement during the next fiscal year in the areas of (1) scientific work stations, (2) personal computing, (3) local area networking, and (4) office automation. Please also indicate standardized sof tware packages for your division.

1. Scientific Work Stations: None planned.

2. Personal Computing: None planned.

3. Local Area Networking: None planned.

4. Office Automation: None planned.

5. Standardized Software: None indicated. 


\section{Argonne West Reactor Program Services Division-.Supply (Cost Center 538)}

\begin{tabular}{|ccccc|}
\hline \multicolumn{5}{c|}{ Primary Contacts } \\
Contact Person & Subject & Fax & Phone & E-Mail Address \\
J.S. Molen & All & 7738 & 7484 & N/A \\
\hline
\end{tabular}

Personal Computers and Workstations

$\begin{array}{cccc} & \text { Existing } & \text { Existing } & \text { Estimated Total } \\ \text { Manufacturer } & \text { FY1991 } & \text { FY1992 } & \text { FY1993 }\end{array}$

$\begin{array}{llll}\text { Altec-286 } & 1 & 1 & 3 \\ \text { H.P. Laser Jet III } & 1 & 1 & 1 \\ \text { PC USA } & 0 & 4 & 4 \\ \text { IBM } & 0 & 1 & 1 \\ \text { MTEK } & 0 & 1 & 1\end{array}$

\section{Word Processors}

Manufacturer

Existing

FY1991

Existing

FY1992

Estimated Total FY 1993

Xerox

1

1

1

\section{Software}

Word Processing/Editing

WordPerfeci

DOS 5

(2)

(2)

Other

List and Mail Plus

Formfiller

49 CFR Reg Scan

29 CFR Reg Scan

2.0 Reg Scan

Communication/Terminal Emulation

Kermit
Database

DBase III Plus

DBase IV

Fox Pro

(1)

(1)

(1)

Utilities
(1)

(1)

(1)

(6)
Disc Optimizer

Windows

Vice Versa

Reflections

$\begin{array}{lc}\text { (1) } & \text { Utilities } \\ \text { (1) } & \text { Disc Optimizer } \\ \text { (1) } & \text { Windows } \\ \text { (1) } & \text { Vice Versa } \\ \text { (2) } & \text { Reflections }\end{array}$

(1) 
Other

$\begin{array}{cccc} & \text { Existing } & \text { Existing } & \text { Estimated Total } \\ \text { Manufacturer } & \text { FY1991 } & \text { FY1992 } & \text { FY1993 }\end{array}$

H.P. Laser Jet III

Please describe systems andior applications that you plan to implement during the next fiscal year in the areas of (1) scientific mork stations, (2) personal computing. (3) local area networking, and (4) office automation. Please also indicale standardized sof fware packages for your division.

1. Scientific Work Stations: None planned.

2. Personal Computing: Implement IBM-compatible personal computers.

3. Local Area Networking: None planned.

4. Office Automation: Upgrade from Xerox to IBM-compatible system.

5. Standardized Software: None indicated. 


\section{Argonne West--Site Engineering/Project Management (Cost Center 556)}

\begin{tabular}{|lcccc|}
\hline \multicolumn{5}{c|}{ Primary Contacts } \\
Contact Person & Subject & Fax\# & Phone & E-Mail Address \\
& & & & \\
John K. Ammon & All & 7656 & 7507 & N/A \\
Gene K. Sherman & All & 7344 & 7353 & N/A \\
Roger L. Black & All & 7656 & 7199 & N/A \\
\hline
\end{tabular}

\begin{tabular}{|lccc|}
\hline & Personal Computers & and & \\
& Existing & Existing & \\
& FY1991 & EYI992 & Estimuted Total \\
FYI993 \\
Manufacturer & 4 & 5 & 5 \\
IBM (AT, XT) & 6 & 7 & 9 \\
Apple (II, Mac, CX) & 1 & 2 & 2 \\
Compaq & 20 & 21 & 18 \\
Club American & 1 & 1 & 0 \\
Intergraph Interact 32c & 15 & 15 & 15 \\
A.C.T (386-33) & 13 & 10 & 10 \\
Gateway & 1 & 2 & 2 \\
M.I.S.(486-330 & 1 & 1 & 1 \\
Z.I.P. (386-25) & 1 & 1 & 1 \\
Dauphin Laptop (386-sx) & 2 & 2 & 13 \\
S \& M (386-25) & 0 & 9 & \\
PC USA & & & 13 \\
\hline
\end{tabular}

\begin{tabular}{|cccc|}
\hline & Word Processors & & \\
& Existing & Existing & Estimated Total \\
Manufacturer & FY1991 & FY1992 & FY 1993 \\
SilverReed & 1 & 1 & 1 \\
\hline
\end{tabular}

\begin{tabular}{|cccc|}
\hline & Software & & \\
& & \multicolumn{2}{|c|}{ Communication/Terminal Emulation } \\
Project Management & $(1)$ & Procomm Plus & $(1)$ \\
TimeLine 4.0 & $(10)$ & DOECOMM & $(3)$ \\
Pertmaster Advance & $(1)$ & Kermit & $(5)$ \\
Expedition & $(2)$ & Carbon Copy & $(1)$ \\
Primavera P3 & & Scientific & \\
& $(1)$ & MathCAD & $(3)$ \\
Desktop Publishing & $(1)$ & Surfer & $(1)$ \\
PageMaker & & & \\
Express & & & \\
\end{tabular}


Software (continued)

$\begin{array}{ll}\text { Graphics } & \\ \text { MacPaint } & (1) \\ \text { ChartMaster } & (1) \\ \text { CricketDraw } & (1) \\ \text { EPS Illustrations } & (1) \\ \text { Paste Ease } & (1) \\ \text { SuperPaint } & (1) \\ \text { Adobe Illustrator } & (1) \\ \text { Impact Imagges } & (1) \\ \text { MacDraft } & (2) \\ \text { MacDraw } & (1) \\ \text { CricketPresent } & (1) \\ \text { CricketGraph } & (1) \\ \text { Power Point } & (1) \\ \text { Hijaak } & (1) \\ & \\ \text { Spreadsheet } & \\ \text { Framework III } & \text { (1) } \\ \text { Quattro Pro } & (4) \\ \text { Works } & (3)\end{array}$

Other

AWORKS

Coach

Grammitik
(1)

(1)

(1)

(1)

(1)

(1)

(4)

(1)

(10)

\section{CAD/CAM}

Microstation 32

AutoCAD Release 10

AutoCAD Release 11

AutoCAD Release 12

(3)

(3)

Word Processing/Editing Word

MacWrite

(4)

WordPerfect 5.1

(2)

(21)

Database

DBase III

(2)

DBase III+

Excel

Utilities

Norton 5.0

MAC Tools

Virex

Networking

Novell Netware 386

V3.11

(100 users)

\section{Network Connections}

\section{Existing \\ FY1992}

Local AppleTalk

Novell Netware 386

Lab-wide Tellabs
Estimated Total

FY1993

$\begin{array}{rr}4 & 7 \\ 40 & 55 \\ 6 & 7\end{array}$


Please describe systems and/or applications that you plan to implement during the next fiscal year in the areas of (1) scientific work stations, (2) personal computing. (3) local area networking, and (4) office automation. Please also indicate standardized software packages for your division.

1. Scientific Work Stations: None planned at the present, but will continue to evaluate their value as an engineerring platform and drafting tool. We are looking at a large format scanning system to convert older handdrawn drawings to the AutoCAD format.

2. Personal Computing: We will increase both in the quantity and use of personal computers. We will look at using one for the tracking of tasks and resources. We have increased the amount and size of databases and are making them available to all personnel.

3. Local Area Networking: We will increase the number of users once we have upgraded to the current version of Novell and will look at bridging to other LANs. There are currently 5 Novell LANs on site that are connected together.

4. Office Automation: CDOCS (Classsified Document Control System) is and will be expanded and used for storage of all records. We have added a second system to speed up the scanning process. We are converting all of our forms to WordPerfect files. This will allow each user to create his own form at a faster rate.

5. Standardized Software: We have adopted AutoCAD as our CAD software, DBase as our database software and WordPerfect as our word processing software. Norton Utilities, Norton Commander, Slick, and Fastback will become our utility software standards. The only group who uses a wide variety of software is Graphics Arts, and this can be attributed to the diverse work they perform. 


\section{Biological and Medical Research (Cost Center 110)}

\begin{tabular}{|ccccc|}
\hline \multicolumn{3}{c|}{ Primary Contacts } \\
Consact Person & Subject & Fax\# & Phone & E-Mivil Address \\
Ken Groh & ADAC & & 3861 & GROH@ANLBEM \\
Carol Fox & Inventory & & 3945 & FOX@ANBIOR \\
\hline
\end{tabular}

\section{Personal Computers and Workstations}

Manufacturer

IBM (PC, AT, XT, PS/2)

Apple (II, Macintosh)

ADAC

MICROLAB

DIGITAL

AST Research

Hewleu Packard

Waters Assoc

DTK 386SX

3 Com 4015

Microstar 286

Microstar 386

Compaq

DIGITAL MicrovaxII

DIGITAL 3100 Model 76

DIGITAL 3100 Model 38

Sun SPARC 2

Sun IPC

MicroStar 486

DIGITAL 4000 Model 60

Turbo Next Station

Silicon Graphics

Next Station

\section{Existing}

TY199]

20

7

1

0

6

7

4

0

6

1

1

1

2

2

1

1

0

0

0

0

0

0

0
Existing

FY1992

25
6
1
0
0
7
4
0
8
1
2
1
3
2
0
1
3
2
1
1
1
1
1

Estimated Total FYl993

23
6
1
0
0
7
4
0
8
1
2
1
3
2
1
1
3
2
1
1
1
1
1

\section{Word Processors}

Manufacturer

IBM

NBI

\section{Existing}

FY1991

5

0
Existing

FY1992

0

0
Estimated Total FY 1993 


\section{Software}

Word Processing/Editing

Text Editor

WordPerfect

Languages/Compilers

BASIC

FORTRAN
(35)

(10)

(10)
Spreadsheet

Lotus 1-2-3

(2)

Utility

Textra

DNASTAR
(1)

(l)

\section{Network Connections}

\section{Existing}

FY1992

Lab-wide Ethernet

DECnet

TCP/IP
50

35

\section{Estimated Total}

FY1993

Please state planned activities andlor applications to be implemented during the next fiscal year in the areas of (1) scientific work stations, (2) personal computing, (3) local area networking, and (4) office automation. Please also indicate standardized software packages for your division.

1. Scientific Work Stations: None planned.

2. Personal Computing: None planned.

3. Local Area Networking: Connect specific PCs to VAX systems for data transfer back and forth.

4. Office Automation: Connect secretarial PCs to each other using PCSA networking system.

5. Standardized Software: WordPerfect 


\section{Building Maintenance (Cost Center 501)}

\begin{tabular}{|ccccc|}
\hline \multicolumn{4}{c|}{ Primary Contacts } \\
Contact Person & Subject & Fax\# & Phone & E-Mail Address \\
Deidre Diei & Workstations & N/A & 5992 & N/A \\
\hline
\end{tabular}

Personal Computers and Workstations

$\begin{array}{cccc}\text { Manufacturer } & \text { Existing } & \text { Existing } & \text { Estimated Total } \\ \text { FY1991 } & \text { FY1993 } \\ \text { IBM (PC, AT, XT, PS/2) } & 10 & 10 & 15\end{array}$

Software

Word Processing/Editing

Word

(4)

Word Perfect

(8)

WordStar

(1)

Database

DBase III Plus

DBase IV

Windows

(3)

(1)

(3)

Graphics

Harvard Graphics

Brady Label

(1)

(2)

Operating Systems

Windows 3.1

Spreadsheet

Quattro Pro

Lotus 1-2-3

(2)

(1)

Utilities

Norton

(2)

Side Kick

(1)

Communication/Terminal Emulation

Procomm Plus

(3) 
Please describe systems andlor applications that you plan to implement during the next fiscal year in the areas of (1) scientific work stations, (2) personal computing, (3) local area networking, and (4) office automation. Please also indicate standardized software packages for your division.

1. Scientific Work Stations: None planned.

2. Personal Computing: Continued expansion of personal computing capabilities is planned for the next year.

3. Local Area Networking: Local area network installation in Buildings $201,4,810,827$ is planned for next year. Expansion of 214 network is planned for the last half of 1992.

4. Office Automation: None planned.

5. Standardized Software: A committee to select and standardize software and hardware system for PFS has been formed. John Mitchell is chairperson, $x$ 8610. The charter of this committce is to maintain consistent computing activities with the PFS Division. 


\section{Central Shops (Cost Center 210)}

Primary Contacts

$\begin{array}{rcccc}\text { Contact Person } & \text { Subject } & \text { Fax } & \text { Phone } & \text { E-Mail Address } \\ \text { Al Schneider } & \text { All } & 6267 & 7082 & \text { N/A }\end{array}$

\section{Personal Computers and Workstations}

Manufacturer

IBM (PC, AT, XT, PS/2)

Compaq

Gateway

Texas Instruments

ANL Electronics 386

PTR Prec. Tec.

\begin{abstract}
Existing
FY1991
\end{abstract}

8
8
2
2
0
0

Existing

FY1992

7
8
1
0
1
1

\section{Estimated Total} FY1993

\section{Software}

Word Processing/Editing

Word

WordPerfect

Communication/Terminal Emulation

Kermit

AZPC2

MSE-Estimating

Spreadsheet

Quattro Pro

(7)
Database

$$
\text { DBase IV }
$$

Mfg. Shop Control

(10)

CAE/CAD/CAM

AutoCAD

SmartCAM

\section{Network Connections}

Existing

FY1992

Novell

Direct Numeric Control (DNC)
10

2

\section{Estimated Total FY1993}

7

8

2

0

1 
Please describe systems and/or applications that you plan to implement during the next fiscal year in the areas of (1) scientific work stations, (2) personal computing, (3) local area networking, and (4) office automation. Please also indicate standardized software packages for your division.

1. Scientific Work Stations: None planned.

2. Personal Computing: None planned.

3. Local Area Networking: None planned.

4. Office Automation: None planned.

5. Standardized Software: None indicated. 


\section{Chemical Technology (Cost Centers 107 \& 269)}

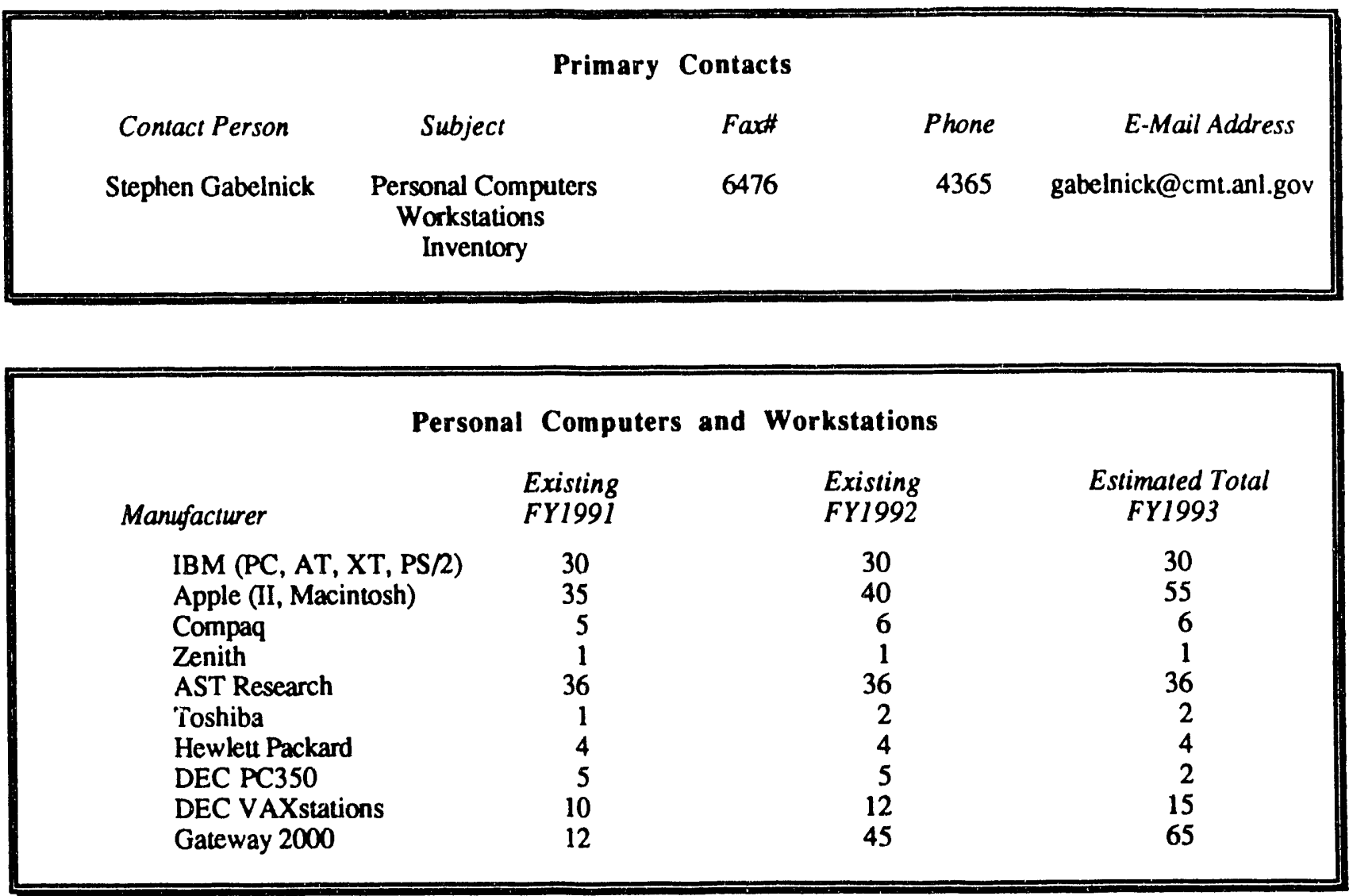

Software

Graphics

MacDraw II

SuperChart

SuperImage

Mass-11 Draw

Cricket Graph

Corel Draw

Power Point

Persuasion

Languages/Compilers

Turbo Pascal

FORTRAN

Basic

C

Utilities

Norton Utilities

PC Tools

Diskfit
(4)

(15)
Communication/Terminal Emulation

VTerm

SmarTerm 240

Reflection

Kermit

Pathworks

Word Processing/Editing

Word

KEDIT

Mass-11

WordPerfect

Desktop Publishing

IBM Interleaf

Expressionist

PageMaker

(1)

(4)

(3)

CAE/CAD

AutoCAD

(3) 


\section{Software (continued)}

Project Management

Timeline

MS Project

Integrated Packages

Excel

Lotus 1-2-3
(2)
Database

dBASE III Plus

Paradox

Other

Desquiew

\section{Network Connections}

Existing
FY1992

Lab-wide Ethernet

DECnet

Both

Lab-wide AppleTalk
80

80

80

40
Estimated Total FY1993

120

120

120

Please describe systems andlor applications that you plan to implement during the next fiscal year in the areas of $(1)$ scientific work stations, (2) personal computing. (3) local area networking, and (4) office automation. Please also indicate standardized software packages for your division.

1. Scientific Work Stations: IBM RISC/6000 for computational chemistry, HP 400 for analytical chemistry.

2. Personal Computing: Continued purchase of PC clones and Macintoshes for office automation.

3. Local Area Networking: Installation of twisted-pair (10 Base-T) Ethernet hub in addition to existing thin wire and thick wire. Installation of CISCO router/bridge, fiber optic Ethernet.

4. Office Automation: WordPerfect Office, PCNAX Gateways.

5. Standardized Software: WordPerfect on VMS, DOS, Macintosh; WordPerfect Office; Excel. 


\section{Chemistry (Cost Center 120)}

\begin{tabular}{|ccccc|}
\hline \multicolumn{5}{c|}{ Primary Contacts } \\
Contact Person & Subject & Fax\# & Phone & E-Mail Address \\
Ron Van Buskirk & All & 3822 & 3684 & vanbuskirk@anl.gov \\
\hline
\end{tabular}

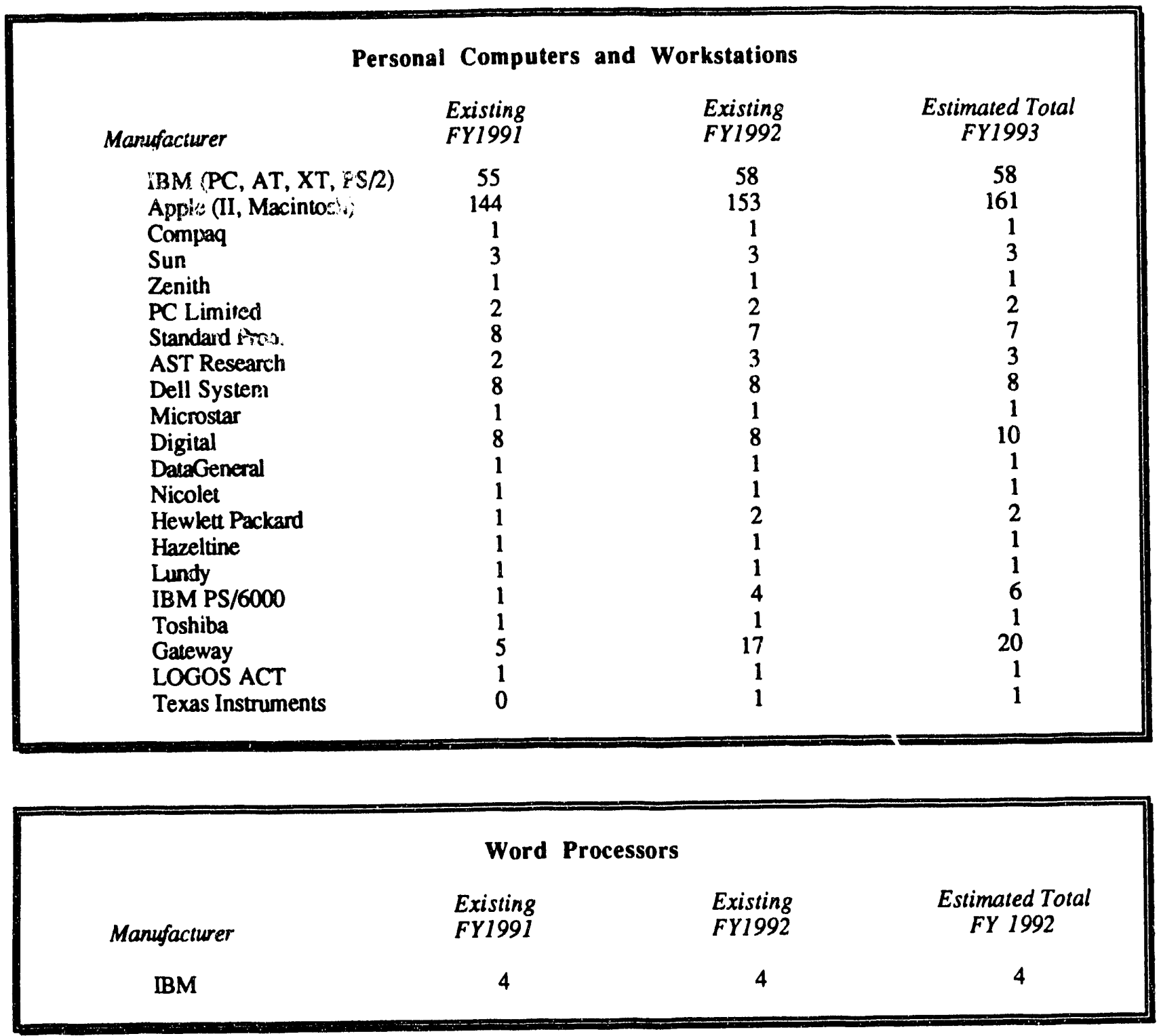




\section{Software}

Languages/Compilers

IBM Basic

IBM Fortran

(7)

LS C

LS Pascal

MacBasic

MS Quick Basic

MS C

MS Quick C

QuickBasic

MacFortran

Turbo Pascal

MacTran Plus

MS Fortran

Communication/Terminal Emulation

Emutek

MacTerminal

PC/Intercomm

PC Talk

SmartTerm

Versaterm

Kermit

NCSA Telnet

(2)

(2)

\section{Database}

DBase IV

WingZ

Double Helix

Foxbase

Filemaker

DataPerfect

(4)

\section{CAE/CAD}

McCad SCM-1

McCad SMT-1

(1)

(1)

Generic CAD

(3)

Claris CAD

(2)

Spreadsheet
Lotus 1-2-3
PC Calc
Excel
Insegrated Packages
SAS

(7)

(2)
Graphics

ANSI Graph

Charting Gallery

CHemDraw

CricketDraw

CriketGraph

ExpressGraph

Framework II

HP Drawgal

Kaleidagraph

MacDraft

MacPlot

PC Plot

TechGraph PD

MacDraw

(1)

(1)

(4)

(3)

(1)

(1)

(1)

(13)

(7)

(1)

(8)

(2)

Word Processing/Editing

Chem Text

WordPerfect

(1)

WordStar

WordUp

Endnote

PC Write

MS Word

Correct Grammar

MacWrite

Utilities

Norton Advanced Utilities

(1)

Norton Commander

Sidekick

PC Tools

(2)

(2)

MacTools

Desktop Publishing

Ready Set Go

(5)

Norton DT for Windows

Publish It Easy

Project Management

Harvard Project Manager

SuperProject

(1)

Mac Project 
Software (continued)

Other

Alchemy

Disk Optimizer

EG\&G Ace

Expressionist

FastBack

First Aid

Halo

Higgins

HP UV/VIS

IGOR

MacEQN

MacRabbit

Mathematica

MDC II

MGM Station

Mince

Mol. Present

Mouse Perfect

LabView

MS Macro ASM
(1)

(1)

(2)

(11)

(1)

(1)

(3)

(1)

(1)

(3)

(1)

(2)

(2)

(1)

(1)

(1)

(2)

(1)

(4)

(1)
MS Windows

MS Works

Natl. Inst. Dev. Pkg.

NB488

Norton for Mac

Passage

PC Model

ProFile

Q\&A

Readability

Reflex Plus

Semantic

Silver Lining

Software Carousel

Super Laser Spool

Trumpet

VTEK

Windows 286

Windows 386

Windows 3.0
(3)

(1)

(6)

(1)

(6)

(1)

(1)

(1)

(1)

(1)

(2)

(1)

(5)

(1)

(1)

(1)

(1)

(1)

(3)

(2)

Network Connections

Existing

FY1992

Lab-wide Ethernet

TCP/IP

DECnet

Both

Lab-wide AppleTalk

Local AppleTalk
63

34

11

55

50
Estimated Total

FY1993 
Please describe systems andlor applications that you plan to implement during the next fiscal year in the areas of (1) scientific work stations, (2) personal computing, (3) local area networking, and (4) office automation. Please also indicate standardized software packages for your division.

I. Scientific Work Stations: None planned.

2. Personal Computing: None planned.

3. Local Area Networking: None planned.

4. Office Automation: None planned.

5. Standardized Software: None indicated. 
Chief Operations Officer (Cost Center 202)

\begin{tabular}{|ccccc|}
\hline & \multicolumn{4}{c|}{ Primary Contacts } \\
Contact Person & Subject & Fax\# & Phone & E-Mail Address \\
Dennis Karras & All & 2948 & 3030 & N/A \\
\hline
\end{tabular}

Personal Computers and Workstations

$\begin{array}{cccc}\text { Manufacturer } & \text { Existing } & \text { Existing } & \text { Estimated Total } \\ \text { FYI991 } & \text { FY1992 } & \\ \text { IBM } & 14 & 14 & 13 \\ \text { MacIntosh } & 6 & 6 & 9 \\ \text { IBM Clone } & 0 & 1 & 1\end{array}$

\section{Software}

Word Processing/Editing

Microsoft Word 5.0

(6)

WordPerfect

(3)

Network NBI Oasys System

Database

DBase II+

DBase IV

(3)

(2)
Other

$$
\text { pmate2 }
$$

Lexis

Westlaw

Foreign Travel Management

(4)

(4)

(1)

(2)

Utilities

Microsoft Word Power Point for Windows 3.0

\section{Network Connections}

\section{Existing}

FY1992

NBI Oasys

Novell
35

2

\section{Estimated Total} FY1993 
Please describe systems and/or applications that you plan to implement during the next fiscal year in the areas of (1) scientific nork stations, (2) personal computing, (3) local area networking, and (4) office automation. Please also indicate standardized software packages for your division.

1. Scientific Work Stations: None planned.

2. Personal Computing: Expand software (WordPerfect, database) and knowledge.

3. Local Area Networking: We are planning a network between OPS and OTD.

4. Office Automation: Be on a network.

5. Standardized Software: Remove NBI. 
Computing and Telecommunications Division (Cost Centers 146, 245, 247, and 296)

\begin{tabular}{|ccccc|}
\hline \multicolumn{5}{c|}{ Primary Contacts } \\
Contact Person & Subject & Fax\# & Phone & E-Mail Address \\
Sally Ott & $\begin{array}{c}\text { Inventory } \\
\text { Jiln Regula }\end{array}$ & 5983 & 2537 & B11807 AT ANLVM \\
Workstations (IBM) & 5983 & 4817 & regula@ani.gov \\
Doug Engert & $\begin{array}{c}\text { LAN Manager Network } \\
\text { Workstations (Sun) }\end{array}$ & 5983 & 5444 & deengen@anl.gov \\
\hline
\end{tabular}

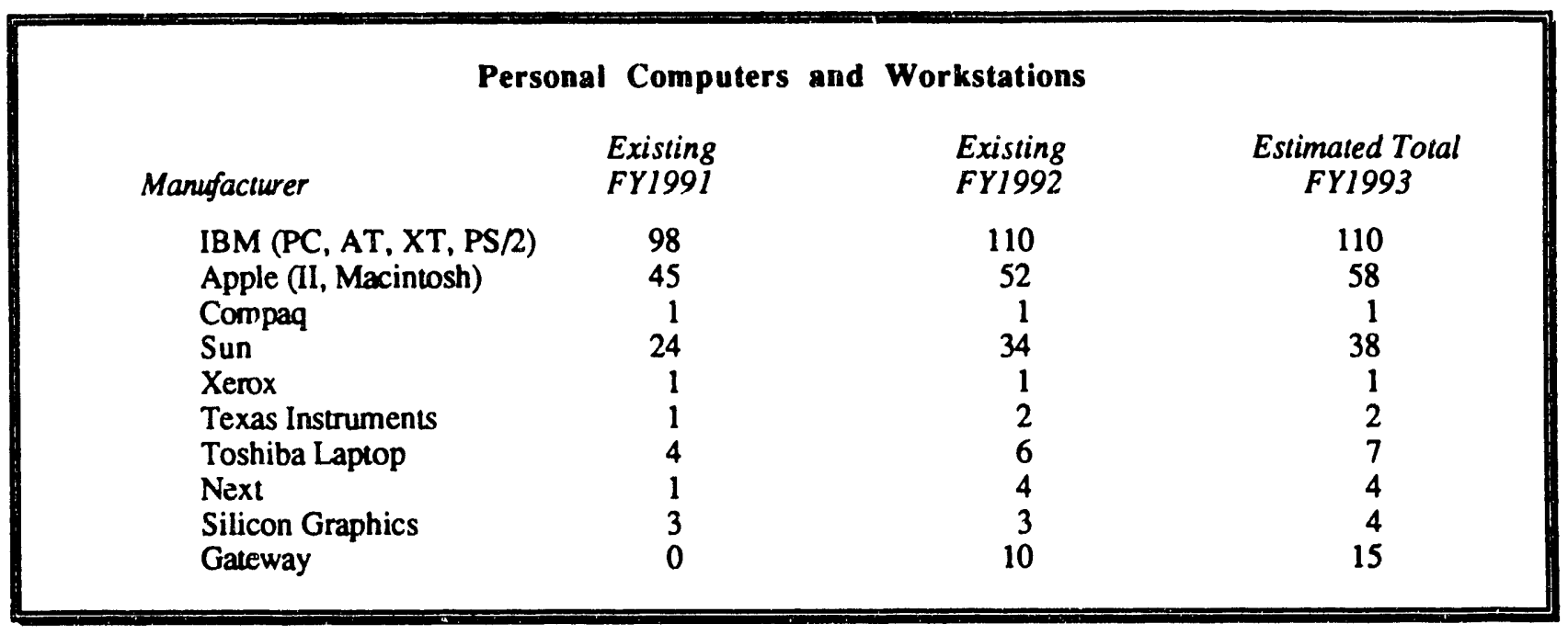

\begin{tabular}{|cccc|}
\hline & Software & & \\
Word Processing/Editing & & Graphics & MacDraw II \\
MacWrite & $(9)$ & MacPaint & $(6)$ \\
Word & $(38)$ & Gem Draw & $(5)$ \\
KEDIT & $(19)$ & SuperImage & $(8)$ \\
Text Editor & $(20)$ & Desktop Publishing & \\
Database & & PageMaker & $(3)$ \\
DBase III Plus & $(15)$ & Project Management & $(10)$ \\
Spieadsheet & & MacProject II & \\
Multiplan & $(12)$ & Communication/Terminal Emulation \\
Integrased Packages & & VTerm & $(4)$ \\
Excel & Kermit & $(9)$ \\
Helix & $(24)$ & AZPC 2 & $(3)$ \\
& $(4)$ & IBM PC3270 & $(4)$ \\
& & & \\
\hline
\end{tabular}




\section{Network Connections}

$\begin{array}{lc}\text { Existing } & \text { Estimated Total } \\ \text { FY1992 } & \text { FY1993 }\end{array}$

Lab-wide Ethernet

TCP/IP

DECnet

Lab-wide AppleTalk

Local AppleTalk

LAN Manager

$\begin{array}{rr}150 & 500 \\ 675 & 700 \\ 600 & 800 \\ 30 & 30 \\ 23 & 50\end{array}$

Please describe systems andlor applications that you plan to implement during the next fiscal year in the areas of (1) scientific work stations, (2) personal computing, (3) local area networking, and (4) office automation. Please also indicate standardized software packages for your division.

1. Scientific Work Stations: CTD will be implementing standardized user environments so that many workstations can be efficiently administered and maintained by a single administrator. Kerberos authentication for workstations is currently under evaluation. The AFS file system will implement network file services across wide area networks.

2. Personal Computing: Promote use of MS-Windows.

3. Local Area Networking: CTD will promote interoperability with TCP/IP compatible features for LAN Manager, Novell, and Pathworks networks. A test environment separated by a router from the Lab-wide network will be exercised in determining options for interoperability. Converting from PC networks to UNIX will also be implemented.

4. Office Automation: CTD will make avaliable applications like ANLPHONE foi distributed network environments (e.g., Apple Talk, UNIX, PCs).

5. Standardized Software: Microsoft Word, Microsoft Excel Spreadsheet. 


\section{Custodial (Cost Center 504)}

\begin{tabular}{|ccccc|}
\hline \multicolumn{4}{c|}{ Primary Contacts } \\
Contact Person & Subject & Fax\# & Phone & E-Mail Address \\
Julie McNeil & All & N/A & 2747 & N/A \\
\hline
\end{tabular}

\begin{tabular}{|cccc|}
\hline & Personal Computers and Workstations & \\
& Existing & Existing & Estimated Total \\
Manufacturer & FY1991 & FY1992 & FY1993 \\
Gateway 2000 & 2 & 2 & 2 \\
\hline
\end{tabular}

\begin{tabular}{|c|c|c|c|}
\hline \multicolumn{4}{|c|}{ Software } \\
\hline $\begin{array}{l}\text { Word Processing/Editing } \\
\text { WordPerfect }\end{array}$ & (2) & $\begin{array}{c}\text { Operating Systems } \\
\text { Windows } 3.0\end{array}$ & (2) \\
\hline $\begin{array}{l}\text { Spreadsheet } \\
\text { MS Excel }\end{array}$ & (1) & & \\
\hline
\end{tabular}

Please describe systems and/or applications that you plan to implement during the next fiscal year in the areas of (1) scientific work stations, (2) personal computing. (3) local area networking, and (4) office automation. Please also indicate standardized software packages for your division.

1. Scientific Work Stations: None planned.

2. Personal Computing: Continued expansion of personal computing capabilities is planned for the next year.

3. Local Area Networking: Local area network installation in Buildings $201,4,810,827$ is planned for next year. Expansion of 214 network is planned for the last half of 1992.

4. Office Automation: None planned.

5. Standardized Software: A committee to select and standardize software and hardware system for PFS has been formed. John Mitchell is chairperson, $\times 8610$. The charter of this committee is to maintain consistent computing activities with the PFS Division. 


\section{DIN (Cost Center 509)}

\begin{tabular}{|lcccc|}
\hline \multicolumn{4}{c|}{ Primary Contacts } \\
Contact Person & Subject & Fax\# & Phone & E-Mail Address \\
Dorothy Andrews & All & 2753 & 2752 & piga at anladm2 \\
\hline
\end{tabular}

\begin{tabular}{|cccc|}
\hline & Personal Computers and Workstations & \\
& Existing & Existing & Estimated Total \\
Manufacturer & FY1991 & FY1992 & 2 \\
Epson & 1 & 1 & 2 \\
\hline
\end{tabular}

\begin{tabular}{|c|c|c|c|}
\hline \multicolumn{4}{|c|}{ Software } \\
\hline $\begin{array}{l}\text { Word Processing/Editing } \\
\text { WordPerfect }\end{array}$ & (2) & $\begin{array}{l}\text { Operating Systems } \\
\text { DOS }\end{array}$ & (2) \\
\hline $\begin{array}{l}\text { Database } \\
\text { DBase IV }\end{array}$ & (2) & $\begin{array}{l}\text { Spreadsheet } \\
\text { Lotus 1-2-3 }\end{array}$ & (2) \\
\hline
\end{tabular}

Please de scribe systems andlor applications that you plan to implement during the next fiscal year in the areas of (I) scientific work stations, (2) personal computing. (3) local area networking, and (4) office automation. Please also indicate standardized software packages for your division.

1. Scientific Work Stations: None planned.

2. Personal Computing: Continued expansion of personal computing capabilities is planned for the next year.

3. Local Area Networking: Local area network installation in Buildings $201,4,810,827$ is planned for next year. Expansion of 214 network is planned for the last half of 1992.

4. Office Automation: None planned.

5. Standardized Software: A committee to select and standardize software and hardware system for PFS has been formed. John Mitchell is chairperson, $x 8610$. The charter of this comimittee is to maintain consistent computing activities with the PFS Division. 


\section{Division of Educational Programs (Cost Center 139)}

\begin{tabular}{|ccccc|}
\hline \multicolumn{4}{c|}{ Primary Contacts } \\
Contact Person & Subject & Fax\# & Phone & E-Mail Address \\
Catherine Beles & $\begin{array}{c}\text { Workstations } \\
\text { Networks }\end{array}$ & 3193 & 4817 & cbeles@dep.anl.gov \\
\hline
\end{tabular}

\section{Personal Computers and Workstations}

$\begin{array}{lccc} & \text { Existing } & \text { Existing } & \text { Estimated Total } \\ \text { Manufacturer } & \text { FY1991 } & \text { FY1992 } & 1993 \\ \text { IBM (PC, AT, XT, PS/2) } & 11 & 15 & 1 \\ \text { Compaq } & 1 & 1 & 2 \\ \text { Club American } & 2 & 2 & 9 \\ \text { Tatung } & 9 & 9 & 20 \\ \text { Epson } & 15-20 & 20-25 & 15 \\ \text { Dell } & 0 & 10 & \end{array}$

\section{Software}

Languages/Compilers

Turbo BASIC

Turbo Pascal

Microsoft C

Word Processing/Editing

Word

Word for Windows

Spreadsheet

Supercalc

Quattro Pro

Other

CC: Mail

Xtree
(2)

\section{Graphics}

Super Image

(1)

Harvard Graphics

Corel Draw

(2)

(1)

Desktop Publishing

Ventura

Pagemaker

Database

dBASE III Plus

dBASE IV

Communication/Terminal Emulation

Kermit

(20) 


\section{Network Connections}

Existing

FY1992

Local 3Com

Local LAN-MAN Server
35
Estimated Total FY1993

Please describe systems and/or applications that you plan to implement during the next fiscal year in the areas of (I) scientific work stations, (2) personal computing, (3) local area networking, and (4) office automation. Please also indicate standardized software packages for your division.

1. Scientific Work Stations: None planned.

2. Personal Computing: Continue to upgrade PCs to replace obsolete machines.

3. Local Area Networking: Implement finishing stages of migration from $3 \mathrm{Com}$ to Microsoft LAN Manager. Install cable trays and pull new Ethernet cable.

4. Office Automation: Design a standardized database file system for the entire division, allowing multiple users access to it.

5. Standardized Software: Microsoft Word, dBASE III Plus, Harvard Graphics, Quattro Pro, Microsoft Windows, CC: Mail, Xtree, Norton Utilities, Supercalc. 


\section{Electronics (Cost Center 143)}

\begin{tabular}{|ccccc|}
\hline \multicolumn{5}{|c||}{ Primary Contacts } \\
Contact Person & Subject & Fax\# & Phone & E-Mail Address \\
Dale Travis & All & 4021 & 6964 & anici::travis \\
Loretta Phillips & All & 4021 & 6934 & N/A \\
\hline
\end{tabular}

\begin{tabular}{|cccc|}
\hline & Personal Computers & and Workstations & \\
& Existing & Existing & Estimated Total \\
Manufacturer & FY1991 & FY1992 & FY1993 \\
Next & & & 2 \\
MCI & 2 & 2 & 1 \\
EL Clone & 1 & 1 & 30 \\
Apple & 21 & 29 & 9 \\
Galeway & 3 & 9 & 5 \\
IBM & 17 & 5 & 26 \\
Panasonic & 1 & 25 & 1 \\
SUN & 1 & 1 & 13 \\
DEC & 4 & 12 & 8 \\
Compaq & 7 & 7 & 2 \\
\hline
\end{tabular}

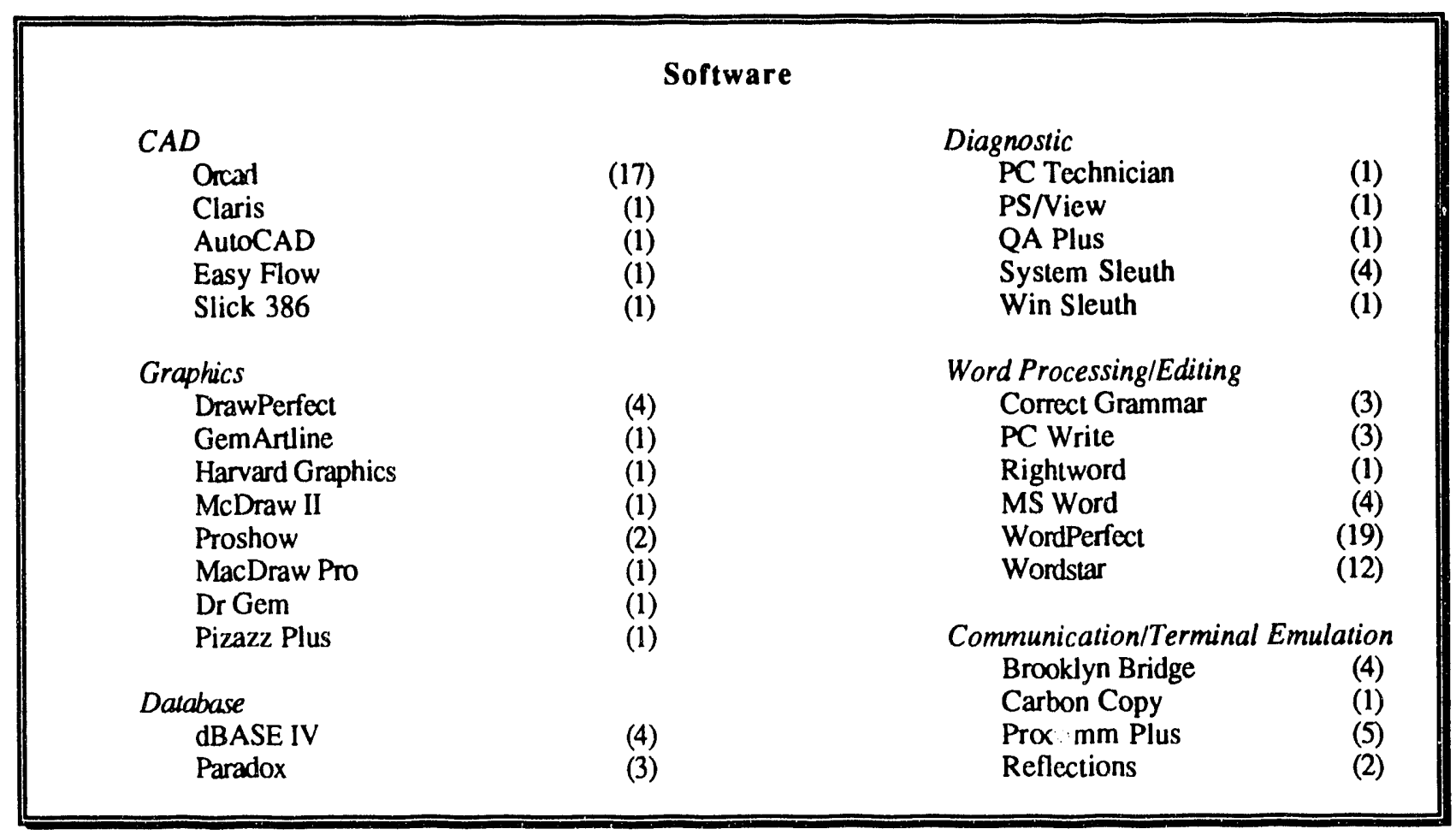




\section{Software (continued)}

Operating System
$3+$ open
DesqView
Direct Access
DR DOS
Gem/3
IBM DOS
LAN Manager
MS DOS
Netware
Norton Desktop
MS Windows
Uniforth Profs
Desqview/X

Spreadsheet
Lotus 1-2-3
MS Excel
Quattro Pro

\section{Utilities}

75 various packages

\section{Network Connections}

Lab-wide Ethernet

\section{Existing}

FY1992

\section{Estimated Total} FY1993

TCP/IP

Telnet

DECNet Vax

Mac Appletalk

IBM PC Clone Pathworks

Terminal Server with LAT

Cisco router

$\begin{array}{rr}9 & 9 \\ 13 & 14 \\ 2 & 7 \\ 5 & 5 \\ 25 & 27 \\ 5 & 5 \\ 1 & 1\end{array}$

Please describe systems andlor applications that you plan to implement during the next fiscal year in the areas of (1) scientific work stations, (2) personal computing, (3) local area networking, and (4) office automation. Please also indicale standardized software packages for your division.

1. Scientific Work Stations: None planned

2. Personal Computing: None planned

3. Local Area Networking: None planned.

4. Office Automation: None planned.

5. Standardized Software: None indicated. 


\section{Energy, Environmental, and Biological Research Program Administration (Cost} Center 274)

\begin{tabular}{|lcccc|}
\hline \multicolumn{5}{c|}{ Primary Contacts } \\
Contact Person & Subject & Fax\# & Phone & E-Mail Address \\
Austine Petersen & Macintosh & 3847 & 3721 & austine_petersen@qmgate.anl.gov \\
\hline
\end{tabular}

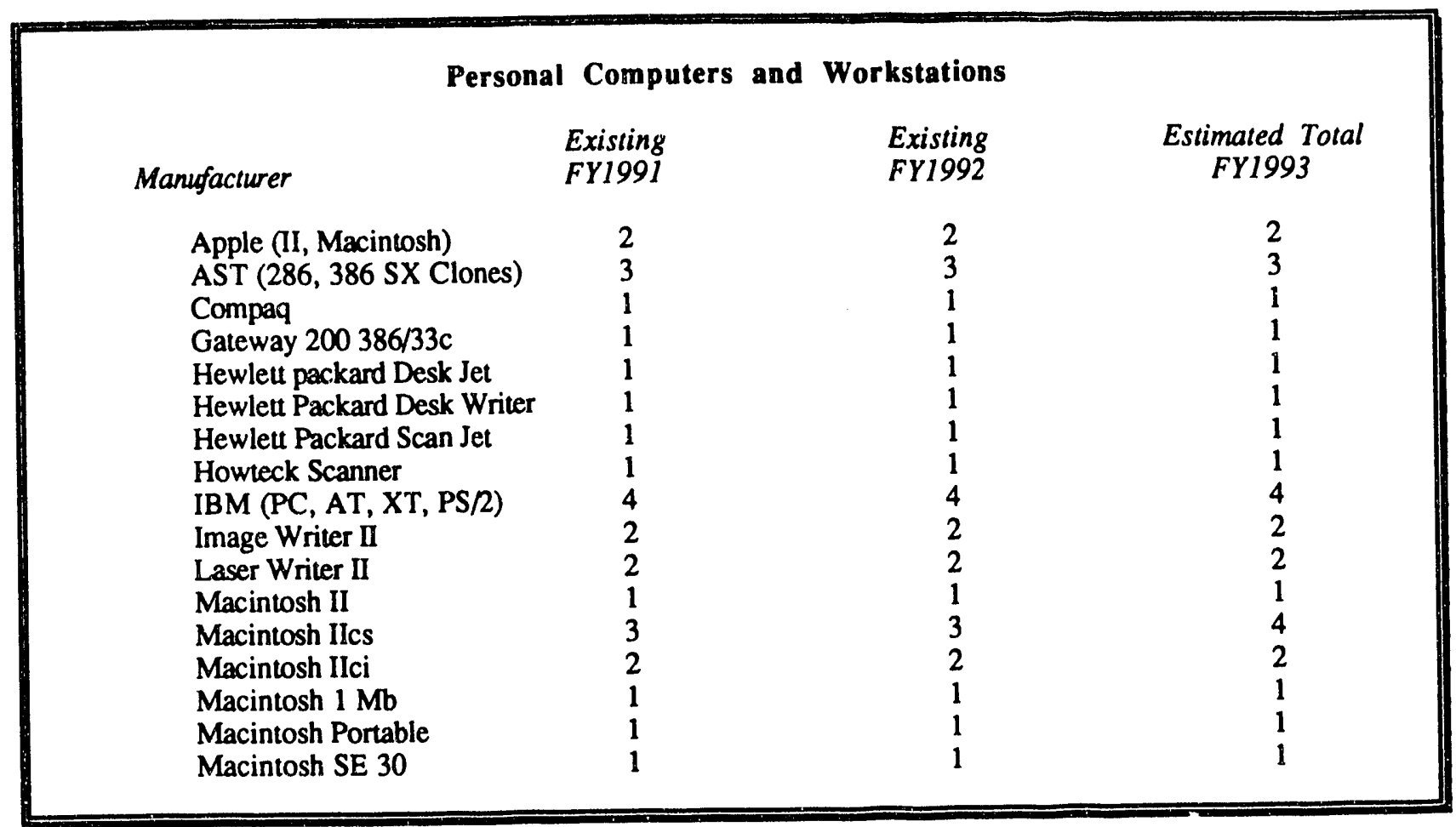

Word Processors

$\begin{array}{cccc}\text { Existing } & \text { Existing } & \text { Estimated Total } \\ \text { Manufacturer } & \text { FY1991 } & \text { FY1992 } & \text { FY } 1992 \\ \text { NBI } & 0 & 0 & 0\end{array}$




\section{Software}

Graphics

Microsoft Chart

Springboard Publisher

PixelPaint

Printshop

MacDraw II

MacProject II

Canvas 2.0

Bisomess 1

Microsoft Power Point

Cricket Graph
(1)

(1)

(1)

(2)

(1)

(1)

(1)

(1)

(1)
Word Processing/Editing

Word

Spreadsheet

Excel

Please describe systems andlor applications that you plan to implement during the next fiscal year in the areas of (1) scientific work stations, (2) personal computing. (3) local area networking, and (4) office automation. Please also indicate standardized software packages for your division.

1. Scientific Work Stations: None planned.

2. Personal Computing: 1 color printer; updates on software; additional hard drives.

3. Local Area Networking: Networking SBC group via Apple Talk.

4. Office Automation: None planned.

5. Standardized Software: Updates as avalaible. 


\section{Energy Systems (Cost Center 155)}

\section{Primary Contacts}

Contact Person

Subject

Fax\#

Phone

E-Mail Address

Erika Shoemaker

All

N/A

7846

erika_shoemaker@qmgate.anl.gov

\section{Personal Computers and Workstations}

All personal computers and workstations are included in the inventory for Cost Center 274.

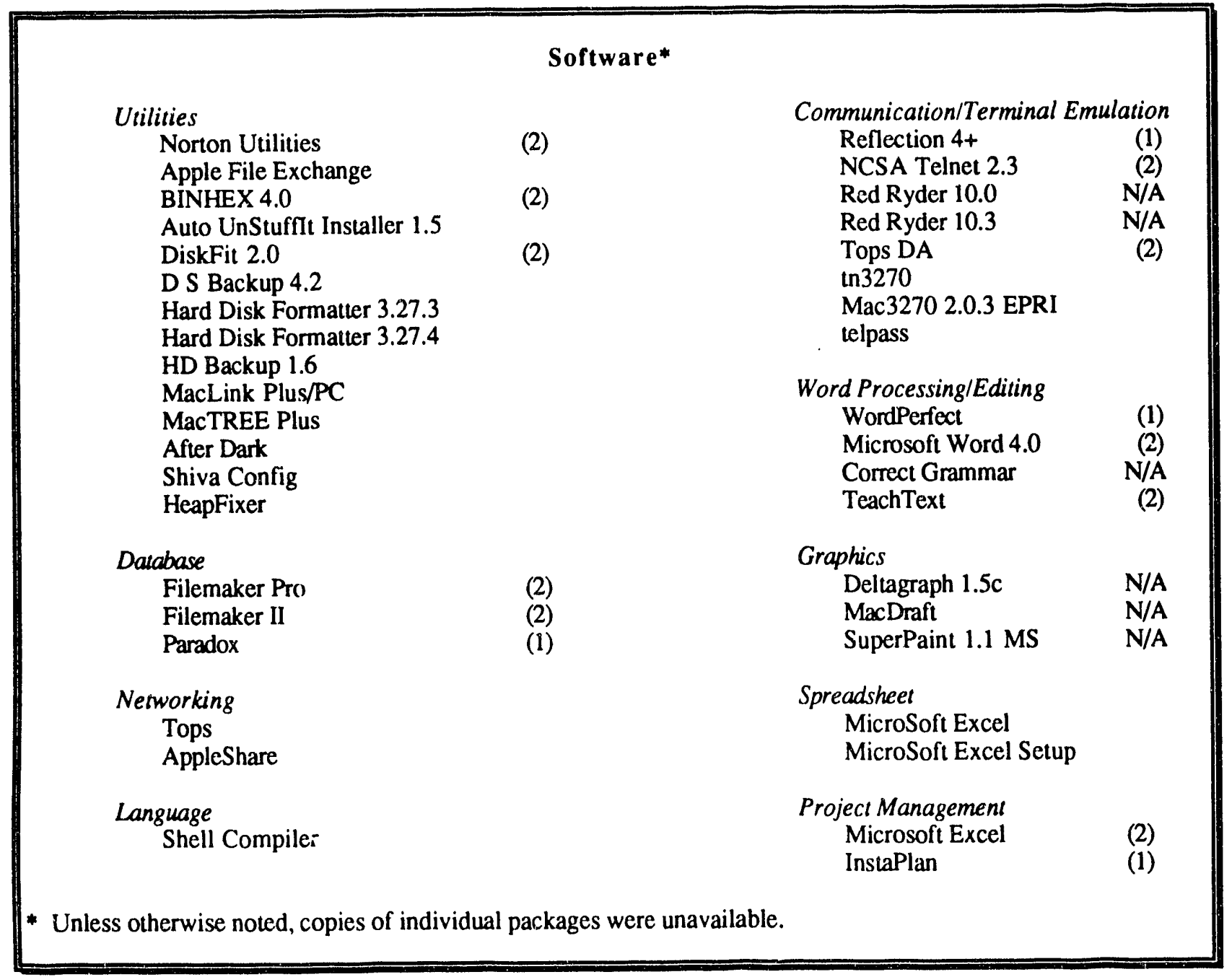


Software (continued)*

Other

DCA Conversion 1.5

Disk First Aid

Edit

Install Keypad

MacWelcome 1.4

PrintMoniter

Speed Disk

(2)

Virus RX

QuickMail

Loodle 3

Smart Alarms

Font/DA Mover

(2)

Font Harmony

(2)

Layout Plus

Offline 2.0

RamDisk

Total Recall 1.2.3

Interbase

DialTrans

Dialog Editor

Expressionist

Installer

Spool

MicroSoft Excel Setup

Profiler

Sound Mover 1.3

Start Tops

QuickDEX

MacEnvy

MailSender

Eraser

Disinfectant 1.8

Font \& Sound Valet

(2)

Word Finder

Interferon 3.1

Oasis 2.0

QM Forms

StuffIt 1.5.1

MacTCP

Appointments

Open PowerStation

Softtalk

Font/DA Mover 3.8

Suitcase

PrintDEX $1.4 \mathrm{e}$

Sound $>$ snd $1.2 e$

UnStuffIt 1.5.1

NetModem

Software Bridge/Mac

Fetch

* Unless otherwise noted, copies of individual packages was unavailable

Please describe systems andlor applications that you plan to implement during the next fiscal year in the areas of (1) scientific work stations, (2) personal computing, (3) local area networking, and (4) office automation. Please also indicate standardized software packages for your division.

1. Scientific Work Stations: None planned.

2. Personal Computing: None planned.

3. Local Area Networking: None planned.

4. Office Automation: None planned.

5. Standardized Software: None indicated. 
Engineering Physics (Cost Centers 115, 117, and 211)

\begin{tabular}{|rcccc|}
\hline \multicolumn{4}{c|}{ Primary Contacts } \\
Contact Person & Subject & Fax\# & Phone & E-Mail Address \\
Charles Miller & All & 5161 & 4564 & N/A \\
\hline
\end{tabular}

\begin{tabular}{|c|c|c|c|}
\hline \multicolumn{4}{|c|}{ Personal Computers and Workstations } \\
\hline Manufacturer & $\begin{array}{l}\text { Existing } \\
\text { FY1991 }\end{array}$ & $\begin{array}{l}\text { Existing } \\
\text { FY1992 }\end{array}$ & $\begin{array}{c}\text { Estimated Total } \\
\text { FY1993 }\end{array}$ \\
\hline $\begin{array}{l}\text { IBM (PC, AT, XT, PS/2) } \\
\text { Apple (II, Macintosh) } \\
\text { Compaq } \\
\text { Sun } \\
\text { Club American } \\
\text { AST Research } \\
\text { MicroStar } \\
\text { Radio Shack } \\
\text { Others } \\
\text { Gateway }\end{array}$ & $\begin{array}{r}53 \\
34 \\
17 \\
11 \\
1 \\
2 \\
5 \\
2 \\
21 \\
39\end{array}$ & $\begin{array}{r}55 \\
36 \\
18 \\
13 \\
1 \\
2 \\
5 \\
2 \\
23 \\
41\end{array}$ & $\begin{array}{r}55 \\
38 \\
19 \\
15 \\
1 \\
2 \\
5 \\
2 \\
23 \\
42\end{array}$ \\
\hline
\end{tabular}

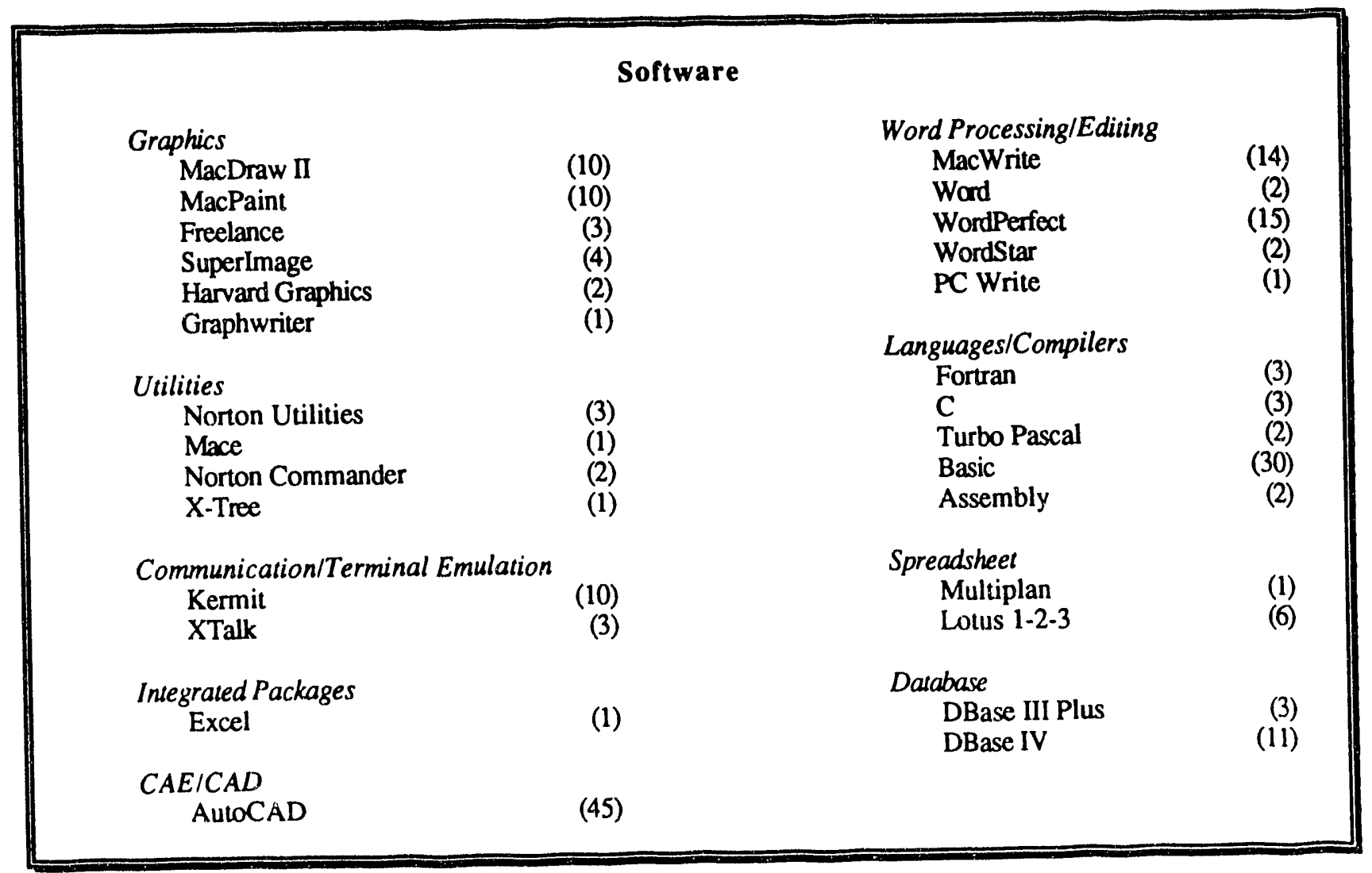




\section{Network Connections}

$\begin{array}{cc}\text { Existing } & \text { Estimated Total } \\ \text { FY1992 } & \text { FY1993 }\end{array}$

Lab-wide Ethernet

TCP/IP

53

55

Local 3Com

6

6

Please describe systems and/or applications that you plan to implement during the next fiscal year in the areas of (1) scientific work stations, (2) personal computing, (3) local area networking, and (4) office automaion. Please also indicate standardized software packages for your division.

1. Scientific Work Stations: Add CAD stations as needed and continue upgrade of older equipment.

2. Personal Computing: Maintain a high level of computing competency in engineering personal computing through improvements in software and computer capabilities.

3. Local Area Networking: Upgrade the Division servers to accommodate more users and implement the use of networking between scientific managers and Division administration.

4. Office Automation: Standardize on IBM (clone) and WordPerfect for admininstrative work.

5. Standardized Software: WordPerfect 5.1 for secretaries on IBM (clones), Lotus 1-2-3 for budgeting, DBase IV. 


\section{Engineering Research (Cost Centers 171 and 271)}

\begin{tabular}{|lccccc|}
\hline \multicolumn{5}{c|}{ Primary Contacts } \\
Contact Person & Subject & Fax\# & Phone & E-Mail Address \\
Ronald Scharping & All & 5318 & 4490 & b11898@anlvm.ctd.anl.gov \\
\hline
\end{tabular}

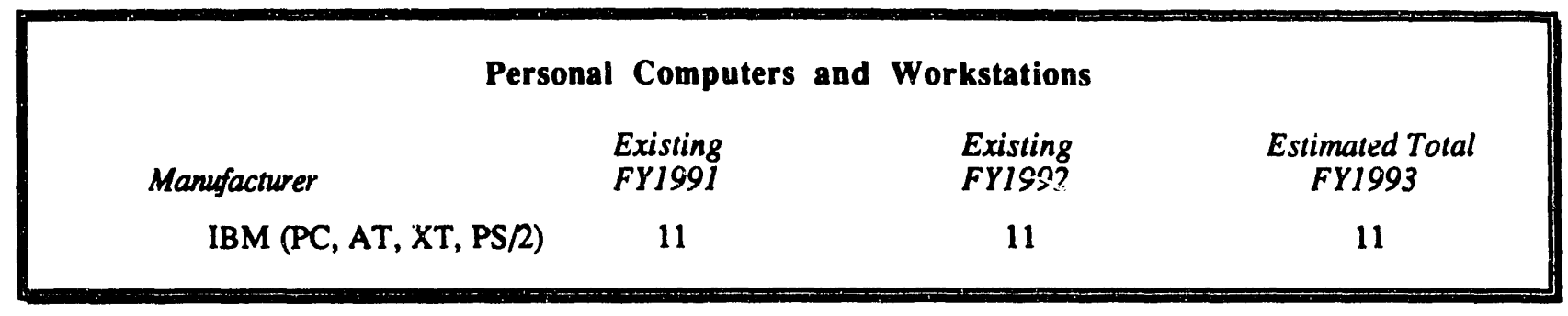

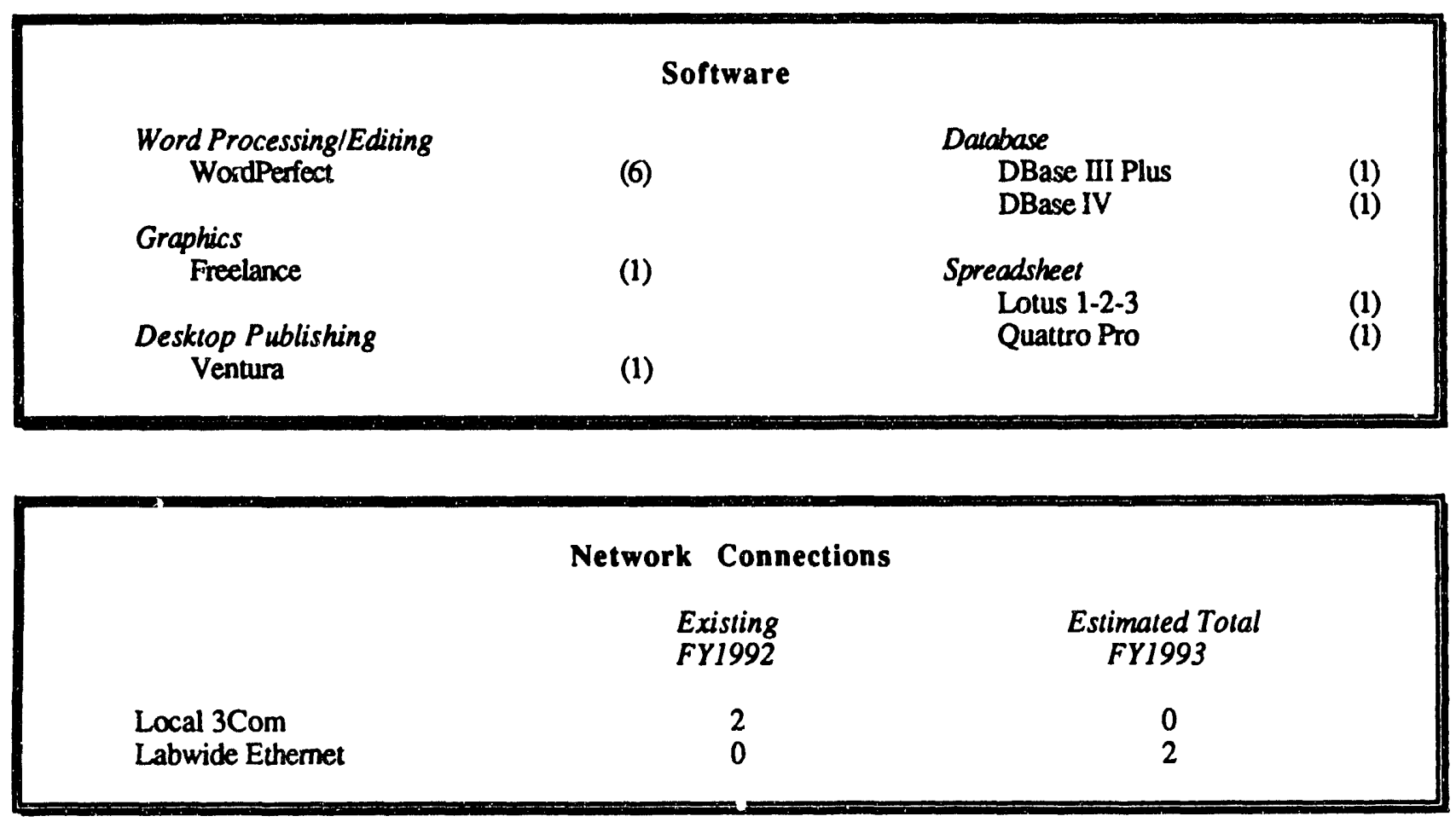


Please describe systems andlor applications that you plan to implement during the next fiscal year in the areas of (I) scientific work stations, (2) personal computing. (3) local area networking, and (4) office automation. Please also indicate standardized software packages for your division.

1. Scientific Work Stations: None planned.

2. Personal Computing: None planned.

3. Local Area Networking: Connection to Labwide Ethernet.

4. Office Automation: None planned.

5. Standardized Software: WordPerfect 5.1. 
Environment, Safety, and Health/Quality Assurance Oversight (Cost Center 333 \& 331)

\begin{tabular}{|ccccc|}
\hline & \multicolumn{3}{c|}{ Primary Contacts } & \\
Contact Person & Subject & Fax\# & Phone & E-Mail Address \\
Dan Miller & All & 5965 & 5775 & Miller@Cygnus.anl.gov \\
\hline
\end{tabular}

\begin{tabular}{|lccc||}
\hline & Personal Computers and Workstations & Estimated Total \\
& Existing & Existing & FY \\
Manufacturer & FY1991 & FY1992 & 4 \\
IBM (PC, AT, XT, PS 2) & 5 & 5 & 6 \\
Gateway 486 & 5 & 7 & 13 \\
Gateway 386 & 3 & 13 & 1 \\
Apple Mac & 1 & 1 & 1 \\
AT\&T Safari & 0 & 1 & \\
\hline
\end{tabular}

\begin{tabular}{|lccc|}
\hline & Word Processors & & Estimated Total \\
& Existing & Existing & FY 1993 \\
Manufacturer & FY199I & FY1992 & 1 \\
Micropolis Radion Array & 0 & 1 & 0 \\
Gateway 486 File & 1 & 1 & 1 \\
Gateway 486 Database & 0 & 1 & 1 \\
Gateway 486 Comm & 1 & 1 & 1 \\
P5 File & 0 & 0 & 1 \\
\hline
\end{tabular}

\section{Software}

Utilities

SQL Windows

dAnalyst

Codebase+t

Phar Lap 386

Borland C++

Netware C 386

Perform Pro

Vulcan

Fox Distribution
(5 users)

(1)

(1)

(1)

(2)

(1)

(1)
Database

$$
\begin{array}{lr}
\text { DBase IV } & \text { (9 users) } \\
\text { Foxpro 2.0 LAN } & \text { (250 users) } \\
\text { SQLbase } & (5 \text { users }) \\
\text { Netware SQL } & (250 \text { users }) \\
\text { Emerald Bay } & (2 \text { users })
\end{array}
$$

Spreadsheet

Lotus 1-2-3

Excel 


\section{Software (continued)}

Communication/Terminal Emulation

On Lan/PC

(15 users)

On Lan/MAC

Procomm Plus

(15 users)

Word Processing/Ecüting

WordPerfect DOS
Graphics

Powerpoint

Harvard

Corel Draw
(4)

(1)

WordPerfect Windows

Network Connections

(Also Support Non-ESH/QA Users)

Existing

FY1992

Novell

DaVinci

Netware Access
40

40

8
Estimated Total FY1993

65-70

65-70

Planned activities andlor applications to be implemented during the next fiscal year in the areas of (1) scientific work stations, (2) personal computing, (3) local area networking, and (4) office automation. Please also indicate standardized software packages for your division.

1. Scientific Work Stations: None planned.

2. Personal Computing: Incremental additions.

3. Local Area Networking: Expand Novell Network.

4. Office Automation: Add on time calendaring program to network.

5. Standardized Software: WordPerfect (DOS, Windows, or Mac). 
Environment, Safety, and Health Division--Division Office

(Cost Center 235)

\begin{tabular}{|lcccc|}
\hline & \multicolumn{4}{c|}{ Primary Contacts } \\
Contact Person & Subject & Fax\# & Phone & E-Mail Address \\
Jeanne Blomquist & All & 5778 & 5640 & N/A \\
\hline
\end{tabular}

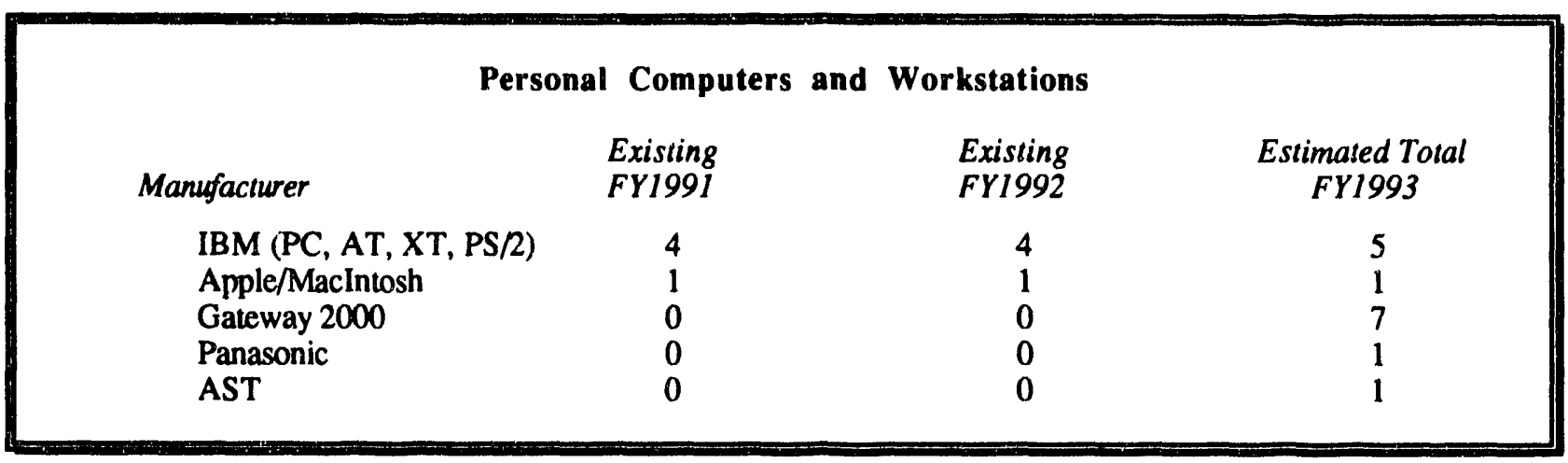

\begin{tabular}{|c|c|c|c|}
\hline \multicolumn{4}{|c|}{ Software } \\
\hline Utilities & \multicolumn{3}{|c|}{ Other } \\
\hline Norton & (2) & Syzygy & (1) \\
\hline Formtool & (2) & Agenda & (1) \\
\hline Deskview 386 & (2) & Grammatik & (1) \\
\hline PC-Kwik & (1) & RESRAD & (1) \\
\hline SYTRAN & (1) & AmiPro & (1) \\
\hline IZZE & (1) & MAC Draft & (1) \\
\hline DeskTop & (1) & MAC Project II & (1) \\
\hline DeskLink & (1) & Calendar Creator Plus & (2) \\
\hline Xtree & (1) & & \\
\hline Perform & (1) & Graphics & \\
\hline Maggepin & (5) & MS Paint & (1) \\
\hline WordLink & (1) & Freelance Graphics & (1) \\
\hline FormTool & (1) & Harvard Graphics & (1) \\
\hline & & Pagemaker & (2) \\
\hline Spreadsheet & & PR2US & (1) \\
\hline Lotus 1-2-3 & (4) & & \\
\hline MultiPlan & (1) & Word Processing/Editing & \\
\hline Quattro Pro & (1) & WordPerfect & (12) \\
\hline Excel & (3) & MultiMate & (1) \\
\hline & & MS-Word & (2) \\
\hline Integrated & & QEDIT & (1) \\
\hline GeoWorks & (1) & & \\
\hline
\end{tabular}




\section{Software (continued)}

Databases

dBASE IV

dBASE III Plus

Paradox

Rbase

FoxPro
(3)

(1)

(2)

(6)

Statistical

SYSTAT
(1)
Communication/Terminal Emulation

$$
\begin{aligned}
& \text { Kermit } \\
& \text { OnLan } \\
& \text { Reflection } \\
& \text { Languages/Compilers } \\
& \text { Quick Basic }
\end{aligned}
$$

(3)

Turbo Pascal

Borland C++

\section{Network Connections}

\section{Existing}

FY1992
Estimated Total

FY1993
Local 3Com

ESH/QA Network
3

Planned activities and/or applications to be implemented during the next fiscal year in the areas of (1) scientific work stations, (2) personal computing, (3) local area networking, and (4) office automation. Please also indicate standardized software packages for your division.

1. Scientific Work Stations: None planned.

2. Personal Computing: None planned.

3. Local Area Networking: All PC's in building 201 will be added to the ESH/QA Network.

4. Office Automation: None planned.

5. Standardized Software: None planned. 


\section{Environment, Safety, and Health Division--Dosimetry and Analytical Services} (External Dosimetry) (Cost Center 235)

\begin{tabular}{|ccccc|}
\hline \multicolumn{4}{c}{ Primary Contacts } \\
Contact Person & Subject & Fax\# & Phone & E-Mail Address \\
Elwyn Dolecek & All & & 3317 & N/A \\
\hline
\end{tabular}

\section{Personal Computers and Workstations}

$\begin{array}{cccc} & \text { Existing } & \text { Existing } & \text { Estimated Total } \\ \text { Manufacturer } & \text { FY1991 } & \text { FY1992 } & \text { FY1993 }\end{array}$

IBM (PC, AT, XT, PS/2)

Gateway
6

0
6

0
7

1

\section{Software}

Word Processing/Editing

Professional Write
Database

dBASE III Plus
(7)

Planned activities andlor applications to be implemented during the next fiscal year in the areas of (1) scientific work stations, (2) personal computing, (3) local area networking, and (4) office automation. Please also include standardized software packages for your division.

1. Scientific Work Stations: None planned.

2. Personal Computing: Norie planned.

3. Local Area Networking: None planned.

4. Office Automation: None planned.

5. Standardized Software: None planned. 


\section{Environment, Safety, and Health Division--Dosimetry and Analytical Services} (Internal Dosimetry) (Cost Center 235)

\begin{tabular}{|ccccc|}
\hline \multicolumn{3}{c}{ Primary Contacts } \\
Contact Person & Subject & Fax\# & Phone & E-Mail Address \\
& & & 3915 & N/A \\
Donald Nelson & All & & 3915 & N/A \\
Richard Holtzman & All & & & \\
\hline
\end{tabular}

Personal Computers and Workstations

Manufacturer

IBM (PC, AT, XT, PS/2)

Hewlet Packard

Gateway 2000

DEC MICROVAX

Epson Equity III Plus

CUI Advantage 286

Hyundai

DEC PDP11/34
Existing

Existing

10

10

Estimated Total FY1993

Software

Utilities

PC Tools

Norton Utilities

Fastback

Sideways

R \& R

Kedit

Screen Extender

Sidekick

XTREE Gold

Spreadsheet

Lotus 1-2-3

Multiplan

Quattro Pro

Excel

Databases

dBASE III Plus

dBASE IV

Quest

(4)

(1)

(1)

(2)

(1)

(1)

(1)

(8)

(2)

(2)

(5)

(1)

Integrated

Enabie
Other

(1)

)

(4)

)

)

AA User
Thermospec
Chemstation
PS Series
Remedy
Cindy
Nuclear Data/Singlet Peak Src
Nuclear Data/Nucl Identifier
Nuclear Data/Spectroscopy App
PCLPAK
AIRDOS-PC (EPA)
Remedy C+
LSTSQ (ANL)
Gofer
CastleSoft
Netroom
LUCAS
ORTEC ADCAM
Control Software
Packard 2200 CA
TRI-CARB 2500
LB4000 Control
SkinDose

(1)

(1)

(2)

(1)

(2)

(5)

(1)

(1)

(1)

(1)

(1)

(3)

(2)

(1)

(1)

(1)

(2)

(1)

(1)

(1)

(1)

(1)

(1) 


\section{Software (continued)}

Other (continued)

CAP88

Camberra Software

Communication/Terminal Emulation

Kermit

Procomm Plus

SIMPC

Statistical

SYSTAT
(2)
Languages/Compilers

Quick Basic

MS Fortran

DEC/MICRO VMS Fortran

Nuclear Data/English Language

(3)

(1)

(1)

(1)

Graphics

Draw Perfect

(1)

Harvard Graphics

Word Processing/Editing WordPerfect

\section{Network Conrections}

Existing
FY1992
Estimated Total

FY1993

Lab-wide Ethernet

DECnet

Local 3Com

2

2

4

4

Planned activities andlor applications to be implemented during the next fiscal year in the areas of (1) scientific work stations, (2) personal computing, (3) local area networking, and (4) office automation. Please also indicate standardized software packages for your division.

1. Scientific Work Stations: None planned.

2. Personal Computing: None planned.

3. Local Area Networking: None planned.

4. Office Automation: None planned. 
Environment, Safety and Health Division--Emergency Management (Cost Center 235)

\begin{tabular}{|ccccc|}
\hline & \multicolumn{3}{c}{ Primary Contacts } \\
Contact Person & Subject & Fax\# & Phone & E-Mail Address \\
Mary Goodkind & All & & 3316 & N/A \\
\hline
\end{tabular}

Personal Computers and Workstations

Manufacturer

IBM (PC, AT,XT, PS/2)

Apple/MacIntosh
Existing
FY1991

1

3
Existing

FY1992

1

3
Estimated Total FY1993

\section{Software}

Utilities

Hypercard

CMS-SCSI

Cameo Workshop

Sam II

MAC Format

Mouse Utilities

MacIntosh Tools

HP Laser Screen Fonts

HP Laser Printing Tools
(2)

(1)

(1)

(1)

(1)

(1)

(1)

(1)

(1)
Graphics

MS Paintbrush

Aldus Persuasion

McDraw PRO

(1)

(1)

(1)

Word Processing/Editing

Word

Professional Write

(2)

(3)

Databases

RBASE for DOS

Cameo
(1)

(3) 
Please describe systems andlor applications that you plan to implement during the next fiscal year in the areas of (1) scientific work stations, (2) personal computing, (3) local area networking, and (4) office automation. Please also indicate standardized software packages for your division.

1. Scientific Work Stations: None planned.

2. Personal Computing: None planned.

3. Local Area Networking: None planned.

4. Office Automation: None planned.

5. Standardized Software: None planned. 


\section{Environment, Safety and Health Division--Fire Protection (Cost Center 235)}

Primary Contacts

Contact Person

Timothy Tess

Subject

Fax\#

All

Personal Computers and Workstations

Manufacturer

$$
\begin{aligned}
& \text { Existing } \\
& \text { FY199I }
\end{aligned}
$$

Existing

FY1992

IBM (PC,AT,XT, PS/2)

Apple/MacIntosh

4

1
Phone

6183
E-Mail Address

N/A

\section{Software}

Word Processing/Editing

MS Word

WordPerfect

MS Publisher

Graphics

HyperCand

MAC Draw II

AutoCad

Utilities

Sam

Sum
(2)

(7)

(1)

(1)

(1)

(1)

(1)

(1)
Other

FPLTool

MS Works

MS Money

MS ENTPAK

HASS

(1)

(1)

(1)

(1)

(1)

Databases

dBASE III Plus

Filemaker Pro

Spreadsheet

MS Excel 
Please describe systems and/or applications that you plan to implement during the next fiscal year in the areas of (1) scientific work stations, (2) personal computing, (3) local area networking, and (4) office automation. Please also indicate standardized software packages for your division.

1. Scientific Work Stations: None planned.

2. Personal Computing: Personal Computer work stations for 8 employees.

3. Local Area Networking: LAN, Novell.

4. Office Automation: A common database which is accessible to all ESH-FP personnel.

5. Standardized Software: dBASE III Plus, MicroSoft Excel. 


\section{Environment, Safety, and Health Division--Health Physics} (Cost Center 235)

\begin{tabular}{|lcccc|}
\hline \multicolumn{4}{c|}{ Primary Contacts } \\
Contact Person & Subject & Fax\# & Phone & E-Mail Address \\
McLouis Robinet & All & & 3326 & N/A \\
\hline
\end{tabular}

\section{Personal Computers and Workstations}

$\begin{array}{lccc} & \begin{array}{c}\text { Existing } \\ \text { Manufacturer }\end{array} & \begin{array}{c}\text { Existing } \\ \text { FY1991 }\end{array} & \begin{array}{c}\text { Estimated Total } \\ \text { FY1993 }\end{array} \\ \text { IBM (PC, AT, XT, PS/2) } & 7 & 7 & 15 \\ \text { Hewlett Packard } & & 1 \\ \text { Gateway 2000 } & & 8 \\ \text { ANL Custom Built } & & 2 \\ \text { DataWork } & & 2 \\ \text { Chicago Computer Exchange Custom Built } & & 6 \\ \text { Compac Plus } & & 1 \\ \text { Zenith } & & 3 \\ \text { NEC } & & 3 \\ \text { LM } & & 1 \\ \text { Tandy } & & 2\end{array}$

\section{Software}

Other

Professional File

NRRPT Exam

Genii

Traces

RSIC Free OAD Codes

ALDOSE

SkyShine

MCNP

Info Select

After Dark

Quartendeck

PC Orps

GSDISP

Remedy

MIRDOSE

VARSKIN

PROIIS

5100

Cameo

Weather Brief

RadDecay
(2)

(1)

(4)
Graphics

Lotus Freelance

AdCADD DTM

(3)

AdCADD INOUT REDUCT

Grapher

PaintBrush

PC Paint

Finger Paint

AutoMap

Drafix + Ultra CAD

AutoCAD

AdCADDCOGO

Generic CAD

Drafix 3D

Drafix Outo

LetterEase

MathCAD

Sygraph

DeskScan

Paintshop Pro

MS Power Point 


\section{Software (continued)}

Other (continued)

Plasma Display Manager

Intel Above Boards

PDS2

(1)

(1)

(1)

Word Processing/Editing

WordPerfect

Professional Write .

MultiMate

Kedit

OmniPage Professional

Communication/Terminal Emulation

Kermit

Procomm

SatisFAXtion

Reflection

(2)

(1)

(1)

Spreadsheet

Quattro Pro

Lotus 1-2-3

(11)

(4)

PFS Pan

Languages/Compilers

MS Fortran

(3)

Quick Basic

(2)

Forth

MS C/C++

Databases

dBASE III Plus

dBASE IV

Paradox

(2)

(2)

(9)

(1)

(10)
Utilities

Formtool

PC MAG Utilities

MicroShield

OAD

Desktop for Paradox

Fasmath Diagnostics

Norton Utilities

AutoChoice

Expert Mouse

Name That Disk

Numerical Recipes C

(3)

(1)

(2)

(2)

(1)

(1)

(2)

(1)

(1)

(1)

Numerical Recipes Fortran

(1)

AIRDOS-EPA

CAP-88

MSoft Mouse

PC Tools

VGA $1024 \mathrm{C}$

Central Point Backup

(1)

(1)

(1)

(1)

(1)

(1)

Company Ladder

(1)

XTree Gold

Chem +

Archie

DeskTeam

GDR (Nucleus)

PCA (Nucleus)

Directory Scanner

(1)

(2)

(1)

(1)

(1)

(1)

(1)

Statistical

Systat
(1)

(1) 
Planned activities and/or applications to be implemented during the next fiscal year in the areas of (1) scientific work stations, (2) personal computing. (3) local area networking, and (4) office automation. Please also indicate standardized software packages for your division.

1. Scientific Work Stations: None planned.

2. Personal Computing: None planned.

3. Local Area Networking: None planned.

4. Office Automation: None planned.

5. Standardized Software: None planned. 
Environment, Safety, and Health Division--Industrial Hygiene (Cost Center 235)

\begin{tabular}{|ccccc|}
\hline \multicolumn{4}{c|}{ Primary Contacts } \\
Contact Person & Subject & Fax\# & Phone & E-Mail Address \\
James Woodring & All & & 5641 & N/A \\
\hline
\end{tabular}

Personal Computers and Workstations

Manufacturer

IBM (PC, AT, XT, PS/2)

Hewlett Packard

Gateway 2000

Panasonic
Existing

Existing

FY1991

4

1
FY1992

4
1
Estimated Total FY1993

\section{Software}

\section{Utilities}

Sideways

Norton

PC Tools

Printshop

Enercharts

Software Bridge

Scanner Utilities

Data Logger Utilities

CNC Utils

Formtool

Word Processing/Editing

WordPerfect

\section{CAE/CAD}

CADD II

Graphics

MS Paint
(1)

(1)

(9)

(1)

(1)

(1)

(1)

(2)

(2)

(1)

(2)

(1)

(1)
Databases

dBASE III Plus
Gloves
CCInfo
Clothing
dBASE IV

(3)

(1)

(1)

(1)

(1)

Communication/Terminal Emulation

Kermit

XTALK

Procomm

(3)

(1)

(2)

Spreadsheet

Lotus 1-2-3

(1)

Languages/Compilers

Basic 


\section{Notuvork Connections}
Existing
FY1992
Estimated Total
FY1993

Local 3Com

5

8

Planned activities andlor applications to be implemented during the next fiscal year in the areas of $(1)$ scientific work stations, (2) personal computing. (3) local area networking, and (4) office automation. Please also indicate standardized sofmare packages for your division.

1. Scientific Work Stations: None planned.

2. Personal Computing: None planned.

3. Local Area Networking: None planned.

4. Office Automation: None planned.

5. Standardized Software: None planned. 
Environment, Safety, and Health Division--Safety Engineering Section (Cost Center 235)

\begin{tabular}{|ccccc|}
\hline \multicolumn{4}{c|}{ Primary Contacts } \\
Contact Person & Subject & Fax\# & Phone & E-Mail Address \\
David Wise & All & & 3836 & N/A \\
\hline
\end{tabular}

\begin{tabular}{|lccc|}
\hline \multicolumn{4}{c|}{ Personal Computers and Workstations } \\
& Existing & Existing & Estimated Total \\
Manufacturer & FY1991 & FY1992 & FY1993 \\
IBM (PC, AT, XT, PS/2) & 2 & 2 & 2 \\
Gateway & 1 & 1 & 3 \\
Packard Bell & 0 & 0 & 1 \\
\hline
\end{tabular}

\begin{tabular}{|llll|}
\hline & Software & & \\
Utilities & & Word Processing/Editing & \\
PC Tools & WordPerfect & (5) \\
PC Anywhere & $(1)$ & Databases & dBASE III Plus \\
$\begin{array}{c}\text { Graphics } \\
\text { Harvard Graphics }\end{array}$ & $(1)$ & Spreadsheet \\
$\begin{array}{c}\text { Communication/Terminal Emulation } \\
\text { Procomm Plus }\end{array}$ & $(2)$ & Excel & (4) \\
& (2) & Other & Text-Trieve \\
& & & (2) \\
\hline
\end{tabular}


Planned activities and/or applications to be implemented during the next fiscal year in the areas of $(1)$ scientific work stations, (2) personal computing, (3) local area networking, and (4) office automation. Please also indicate standardized software packages for your division.

1. Scientific Work Stations: None planned.

2. Personal Computing: None planned.

3. Local Area Networking: All workstations are to be added to ESH 3COM local area network.

4. Office Automation: None planned.

5. Standardized Software: None planned. 


\section{Environment, Safety, and Health Division--Training and Quality Assurance (Cost} Center 235)

\begin{tabular}{|rcccc|}
\hline \multicolumn{4}{c|}{ Primary Contacts } \\
Contact Person & Subject & Faxit & Phone & E-Mail Address \\
Dean Larson & All & & 4573 & N/A \\
\hline
\end{tabular}

\begin{tabular}{|lccc|}
\hline \multicolumn{3}{|c|}{ Personal Computers } & and Workstations \\
& Existing & Existing & Estimated Total \\
Manufacturer & FY1991 & FY1992 & FY1993 \\
IBM (PC, AT, XT, PS/2) & 4 & 4 & 5 \\
Gateway 2000 & & & 4 \\
Sony & & & 1 \\
AST & & 1 \\
Apple/MacIntosh & & 2 \\
\hline
\end{tabular}

\begin{tabular}{|c|c|c|c|}
\hline \multicolumn{4}{|c|}{ Software } \\
\hline Graphics & & Other & \\
\hline HyperCard (Claris) & (1) & Dazzle & (2) \\
\hline Lotus Freelance Plus & (1) & Forth Dimension & (1) \\
\hline Harvard Graphics & (4) & MAC Project II & (1) \\
\hline PictureIt & (4) & IMC & (1) \\
\hline MAC Draw Pro & (1) & Critical Safety & (1) \\
\hline MAC Draft & (1) & TenCore & (4) \\
\hline ORG+ & (2) & Crossword & (1) \\
\hline Super Image & (1) & Metric X MX110 & (1) \\
\hline Print Maker & (1) & RadDecay & (1) \\
\hline VIP & (1) & Certificate Maker & (1) \\
\hline EnerGraphics & (1) & Certificate Library & (1) \\
\hline Hot Shots & (3) & Radiation Worker Safety Course & (2) \\
\hline PhotoMetric & (1) & HazMat Shipping Course & (1) \\
\hline Claris MAC Draw II & (1) & $\begin{array}{l}\text { Generic Rad Worker Safety } \\
\text { Training }\end{array}$ & (1) \\
\hline Word Processing/Editing & & IBM Linkway & (1) \\
\hline WordPerfect & (10) & & \\
\hline MS Word & (1) & Communication/Terminal Emulation & \\
\hline Typist & (1) & QModem & (1) \\
\hline Q\&A & (1) & Kermit & (2) \\
\hline Professional Write & (1) & PC Plus & (1) \\
\hline
\end{tabular}




\section{Software (continued)}

Utilities

PC Tools

Logical Connection

Argosy Software Bridge

Fifth Generation DiscLock

Scan

Soft PC

Grammatic IV

Formtools

SitBack

XTree

CatDisk

Sideways

Norton Utilities

Statistical

Chemistry Works
(8)

(3)

(3)

(1)

(1)

(1)

(1)
Spreadsheet

Claris File Maker Pro

Lotus 1-2-3

Quattro Pro

MultiPlan

(2)

(1)

(2)

(1)

Databases

OETS (Tracking System)

(1)

Video

Regscan

(1)

dBASE III Plus

dBASE IV

(1)

(2)

(2)

Language/Compiler

Clipper

(2)

Basic

Planned activities andlor applications to be implemented during the next fiscal year in the areas of (1) scientific work stations, (2) personal computing, (3) local area networking, and (4) office automation. Please also indicate standardized software packages for your division.

1. Scientific Work Stations: None planned.

2. Personal Computing: None planned.

3. Local Area Networking: None planned.

4. Office Automation: None planned.

5. Standardized Software: None planned. 


\section{Environmental Assessment and Information Sciences Division (Cost Center 165)}

\begin{tabular}{|c|c|c|c|c|}
\hline \multicolumn{5}{|c|}{ Primary Contacts } \\
\hline Contact Person & Subject & Fax\# & Phone & E-Mail Address \\
\hline $\begin{array}{l}\text { Dee Seymour } \\
\text { Lou Kvitek } \\
\text { Dick Eagan } \\
\text { James Renz }\end{array}$ & $\begin{array}{c}\text { IBM } \\
\text { Sun Workstations } \\
\text { Sun Networks } \\
\text { PC and Mac } \\
\text { Networks }\end{array}$ & $\begin{array}{l}5128 \\
5128 \\
5128 \\
5128\end{array}$ & $\begin{array}{l}8023 \\
3132 \\
3435 \\
4650\end{array}$ & $\begin{array}{l}\text { seymour@athens.eid.anl.gov } \\
\text { kvitek@athens.eid.anl.gov } \\
\text { eagan@eid.anl.gov } \\
\text { renz@ athens.eid.anl.gov }\end{array}$ \\
\hline
\end{tabular}

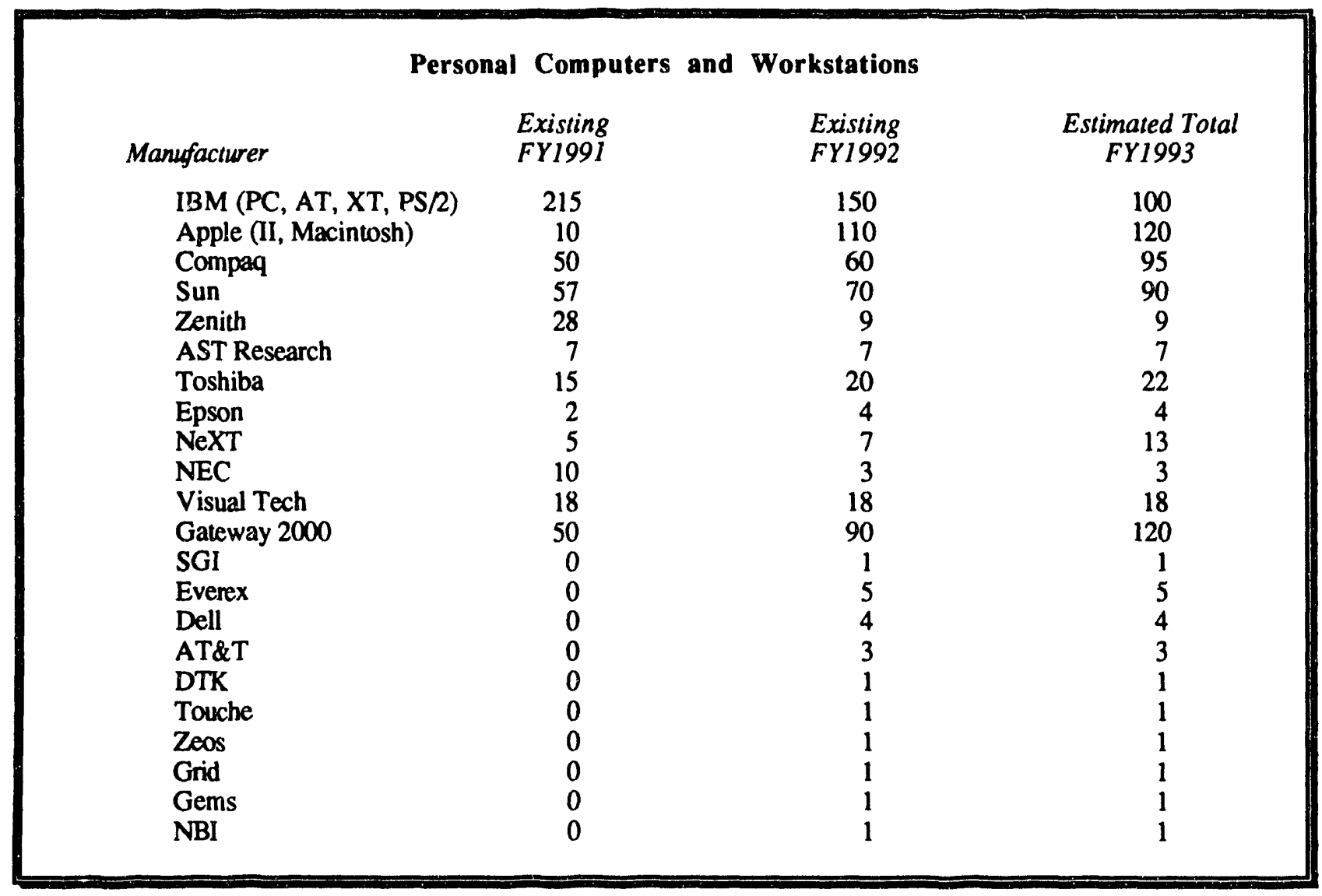




\section{Software}

Communication/Terminal Emulation

VTerm

Procomm

VersaTerm

Red Ryder

Relay Gold

Kermit

SmarTerm

Word Processing/Editing

MacWrite

Word

KEDTT

WordPerfect

Word Star

Norton

Framemaker

\section{CAE/CAD}

AutoCAD

MacDraft

VersaCAD

Desktop Publishing

PageMaker

(6)

Legend

Insegrated Packages

Excel

Lotus 1-2-3
Graphics

MacDraw II

MacPaint

(9)

Gem Draw

(1)

Harvard Graphics

Kaleidagraph

(25)

Graphic

SuperImage

Cricket Graph

Languages/Compilers

MS Fortran

Lahey Fortran

Turbo Pascal

Turbo C

MS C

Database

dBASE

Q\&A

Paradox

Ingris

Project Management MacProject II

Timeline

Utilities

Norton

MS Windows

\section{Network Connections}

Existing

FY1992

Lab-wide Ethernet

TCP/IP

DECnet

Both

Lab-wide AppleTalk

Local 3Com

Novell
375

3

3

144

0

200
Estimated Total FY1993

200

1

150

0 
Please describe systems andlor applications that you plan to implement during the next fiscal year in the areas of (1) scientific work stations, (2) personal computing, (3) local area networking, and (4) office automation. Please also indicate standardized software packages for your division.

1. Scientific Work Stations: Develop and enhance GIS capabilities.

2. Personal Computing: Standardize all computers to latest operating system.

3. Locul Area Networking: Route NOVELL over labwide Ethernet.

4. Office Automation: None planned.

5. Standardized Software: WordPerfect, Microsoft C, Borland C, Lotus, CC:Mail, MSWindows, DOS 5.0, Microsoft Word, MacSystem 7 
Environmental Research Division (Cost Center 149)

\begin{tabular}{|ccccc|}
\hline \multicolumn{4}{c|}{ Primary Contacts } \\
Contact Person & Subject & Fax\# & Phone & E-Mail Address \\
Terry Pawlisz & All & 5747 & 4487 & $\begin{array}{c}\text { terry_pawlisz@qmgate. } \\
\text { mct.anl.gov }\end{array}$ \\
\hline
\end{tabular}

\begin{tabular}{|lccc|}
\hline \multicolumn{4}{c|}{ Personal Computers and Workstations } \\
& Existing & Existing & Estimated Total \\
Manufacturer & FY1991 & FY1992 & FY1993 \\
IBM (PC, AT, XT, PS/2) & 10 & 12 & 12 \\
Apple (II, Macintosh) & 65 & 72 & 80 \\
Zenith & 3 & 3 & 3 \\
ComputAdd & 2 & 2 & 2 \\
Gateway & 1 & 1 & 1 \\
Sun & 1 & 1 & 3 \\
\hline
\end{tabular}

\begin{tabular}{|cccc|}
\hline & Word Processors & (Portable/Lap Tops) \\
& Existing & Existing & Estimated Total \\
Manufacturer & FY1991 & FY1992 & \\
Zenith & 1 & 1 & 1 \\
Dauphin (386) & 1 & 1 & 1 \\
Toshiba & 2 & 2 & 3 \\
MacIntosh & 1 & 1 & 6 \\
Cambridge & 1 & 1 & 1 \\
\hline
\end{tabular}


Software

Languages/Compilers

Fortran

MacFORTRAN

Quicksilver

ANX

Basic

\section{Graphics}

MacDraw II

MacDraft

Delta Graph

SuperPaint

Kaleidagraph

Database

dBASE III Plus

Filemaker (II, Pro)

dBASE IV

Genifer

dBXI

Word t, es ing/Editing

Mach is ice

Microsoft Word

WordPerfect

Write Now

\section{Utilities}

Systat

Norton Utilities

Symantic Utilities

Spreadsheet

Lotus 1-2-3

Microsoft Excel

Presentation Packages

Power Point

(7)

(2)

(1)

(1)

(2)

(5)

(1)

(1)

(1)

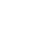

$C A D$

$$
\text { AutoCAD } 386
$$

\section{Other}

MORE II

Calendar Maker

Smart Forms

Adobe Type Manager

The Big Thesaurus

Tennelec

Plink 86

Mac Link Plus Transiators

Smart Labels

TOPS

DiskFit

Math Type

End Note +

Level 5

Word Exchange

Laplink

Micro Planner

SUM II

Fast Forms

Expressionist

Image Grabber

Ortec

4th Dimensiun

Camera

PC Partner

(3)

(5)

(2)

(1)

(1)

(1)

(1)

(1)

(1)

(7)

(3)

(4)

(1)

(1)

(l)

(1)

(1)

(8)

(1)

(1)

(1)

(2)

(1)

(1)

(1)

Communication/Terminal Emulation

VersaTerm (reg, pro)

Red Ryder

NCSA Telnet (site-wide license)

Geographic Information Systems

GRASS

ARC-INFO

Integrated Packaages

EXSYS

(2)

EXSYS Pro

(1) 


\section{Network Connections}

Existing

FY1992

Lab-wide Ethemet

TCP/IP

DECnet
3

9

\section{EstimatedTotal} FY1993

Please describe systems and/or applications that you plan to implement during the next fiscal year in the areas of (I) scientific work stations, (2) personal computing, (3) local area networking, and (4) office automation. Please also indicate standardized software packages for your division.

1. Scientific Work Stations: Using a SUN workstation for advanced seismic processing; upgrading existing SUN for site planning (data integration); using a SUN for field operations in a mobile location.

2. Personal Computing: None planned.

3. Local Area Networking: Networking Macintosh computers with the SUN workstation(s) to build greater compatibility.

4. Office Automation: None planned.

5. Standardized Software: None indicated. 
Environment and Waste Management Program (Cost Center 140)

\begin{tabular}{|ccccc|}
\hline \multicolumn{4}{c|}{ Primary Contacts } \\
Contact Person & Subject & Fax\# & Phone & E-Mail Address \\
Dolores Ray & All & & 5640 & N/A \\
\hline
\end{tabular}

\begin{tabular}{|cccc|}
\hline \multicolumn{4}{c|}{ Personal Compsters and Workstations } \\
& Existing & Existing & Estimated Total \\
Manufacturer & FY1991 & FY1992 & FY1993 \\
IBM (PC, AT, XT, PS/2) & 13 & 13 & 20 \\
Gateway 2000 & 0 & 2 & 2 \\
\hline
\end{tabular}

\begin{tabular}{|c|c|c|c|}
\hline \multicolumn{4}{|c|}{ Software } \\
\hline \multirow{3}{*}{$\begin{array}{l}\text { Word Processing/Editing } \\
\text { KEDIT } \\
\text { WordPerfect }\end{array}$} & \multicolumn{3}{|c|}{ Database } \\
\hline & (1) & DBase III Plus & (16) \\
\hline & (13) & DBase IV & (10) \\
\hline \multirow{4}{*}{$\begin{array}{l}\text { Graphics } \\
\text { Grapher } \\
\text { Surfer }\end{array}$} & & \multicolumn{2}{|c|}{ Communication/Terminal Emulation } \\
\hline & (1) & Kermit & (4) \\
\hline & & Spreadsheet & \\
\hline & & Lotus 1-2-3 & (13) \\
\hline
\end{tabular}

\begin{tabular}{|cc|}
\hline Network Connections & \\
& Existing \\
FY1992 & Estimated Total \\
FY1993 & 30 \\
\hline
\end{tabular}


Please describe systems and/or applications that you plan to implement during the next fiscal year in the areas of $(1)$ scientific work stations, (2) personal computing, (3) local area networking, and (4) office automation. Please also indicate standardized software paikages for your division.

1. Scientific Work Stations: None plarned.

2. Personal Computing: None planned.

3. Local Area Networking: Local area network will be expanded to include all of EWM personnel.

4. Office Automation: NCise planned.

5. Standardized Software: None indicated. 


\section{Facilities Planning/Engineering (Cost Center 512)}

\begin{tabular}{|lcccc|}
\hline & \multicolumn{3}{c|}{ Primary Contacts } \\
Contact Person & Subject & Fax\# & Phone & E-Mail Address \\
Vytenis Milunas & All & & 7336 & N/A \\
\hline
\end{tabular}

\begin{tabular}{|cccc|}
\hline & Personal Computers and Workstations & \\
& Existing & Existing & Estimated Total \\
Manufacturer & FYI991 & FY1992 & FY1993 \\
IBM (PS/2) & 19 & 16 & 16 \\
Compaq & 3 & 5 & 5 \\
Epson & 6 & 10 & 10 \\
Mitac & 1 & 1 & 1 \\
Gateway & 0 & 5 & 10 \\
PCC & 0 & 5 & 5 \\
\hline
\end{tabular}

\section{Software}

\section{$C A E / C A D$}

Precade

AutoCAD 386

MathsoftCAD

AutoCAD 10

AutoCAD 11

ASG Core

ASG Mechanical

ASG Plumbing

ASG Structural

ASG Mechanical HVAC

ASG Electrical

Word Processing/Editing

Multimate

Microsoft Word

Word 5.0

WordPerfect Office

WordPerfect

WordPerfect 5.1

RightWriter

Other

386 Max Professional

OSHA Fast Regs.

Micros-DOE

Procomm
(1)

(1)

(1)

(1)

(1)

(4)
Utilities

Lotus Agenda

Compaq Faststart

Norton Utilities

Norton Commander

Comert

EMCEG

Dapper

Captor

Afault

Cable

Deft

(1)

(1)

(4)

(6)

(1)

(1)

(1)

(1)

(1)

(1)

(1)

Graphics

Harvard Graphics

Perform

Original Charts

DrawPerfect

(1)

(2)

Org +

Creative Calendar

(9)

(1)

Project Mrunagement

Primavera

Cambridge Analyst.

(1)

(1)

Operating Systems

Compaq MS-DOS 


\section{Software}

Spreadsheet

Microsoft Multiplan

Lotus 1-2-3
(1)

(22)
Database

dBASE IV

RBase
(4)

(2)

Network Connections

Existing

FY1992
Estimated Total

FY1993

DecNet

Please describe systems andlor applications that you plan to implement during the next fiscal year in the areas of (1) scientific work stations, (2) personal computing. (3) local area networking, and (4) office automation. Please also indicale standardized software packages for your division.

1. Scientific Work Stations: None planned.

2. Personal Computing: Continued expansion of personal computing capabilities is planned for the next year.

3. Local Area Networking: Local area network installation in Buildings $201,4,810,827$ is planned for next year. Expansion of 214 network is planned for the last half of 1992.

4. Office Automation: None planned.

5. Standardized Software: A committee to select and standardize software and hardware system for PFS has been formed. John Mitchell is chairperson, $\mathbf{x 8 6 1 0}$. The charter of this committee is to maintain consistent computing activities with the PFS Division. 


\section{Fire Department (Cost Center 236)}

Primary Contacts

$\begin{array}{lcccc}\text { Contact Person } & \text { Subject } & \text { Fax\# } & \text { Phone } & \text { E-Mail Address } \\ \text { Cynthia Hijuelos } & \text { Workstations } & 5440 & 6136 & \text { NiA }\end{array}$

Personal Computers and Workstations

$\begin{array}{cccc} & \text { Existing } & \text { Existing } & \text { Estimated Total } \\ \text { Manufacturer } & \text { FY1991 } & \text { FY1992 } & \text { FY1993 } \\ \text { IBM (PC, AT, XT, PS/2) } & 3 & 4 & 4 \\ \text { Apple (II, Macintosh) } & 1 & 3 & 3\end{array}$

\section{Software}

Database

dBASE III

dBASE IV

CAMEO

FireProof (FMS) Tracking System (1)

CAE/CAD

ACAD
(1)

(1)

(1)
Word Processing/Editing
MultiMate
WordPerfect

(1)

Graphics

Microsoft chart
(1)

Please describe systems andlor applications that you plan to implement during the next fiscal year in the areas of (1) scientific work stations, (2) personal computing. (3) local area networking, and (4) office automation. Please also indicate standardized software packages for your division.

1. Scientific Work Stations: None planned.

2. Personal Computing: Computerization of day-to-day emergency response data for rapid retrieval and analyses is planned for the next fiscal year. (Software for this program is undetermined at this date.)

3. Local Area Networking: None planned.

4. Office Automation: None planned.

5. Standardized Software: WordPerfect. 


\section{Health Department (Cost Center 148)}

\section{Primary Contacts}

Contact Person

Subject

Fax\#

Phone

E-Mail Address

Ken Vassallo

All

2804

N/A

Personal Computers and Workstations

Manufacturer

$\begin{array}{ll}\text { Existing } & \text { Existing } \\ \text { FY1991 } & \text { FY1992 }\end{array}$

Estimated Total FY1993

IBM (PC, AT, XT, PS/2)

18

18

20

\begin{tabular}{|lccc|}
\hline & Software & & \\
Word Processing/Editing & & Spreadsheets & Excel \\
Word & $(10)$ & Utility & $(10)$ \\
WordStar & $(4)$ & SideKick & \\
& & SideKick Plus & $(2)$ \\
Database & $(2)$ & Norton 4 & $(1)$ \\
DBase IV & $(1)$ & & $(3)$ \\
Informix & $(1)$ & Other & \\
Clipper & & Free Link Micro & $(8)$ \\
Communication/Terminal Emulation & Stedman's Med. & $(1)$ \\
Kermit & $(2)$ & IBM DOS & $(20)$ \\
IBM PC3270 & $(7)$ & & \\
Telnet & $(1)$ & & \\
\hline
\end{tabular}


Planned activities and/or applications to be implemented during the next fiscal year in the areas of $(1)$ scientific work stations, (2) personal computing, (3) local area networking, and (4) office automation. Please also indicate standardized software packages for your division.

1. Scientific Work Stations: None planned.

2. Personal Computing: Add new PS/2 Workstations to LAN Manager Network.

3. Local Area Networking: Local Area LAN Manager.

4. Office Automation: Barcode, check in process, direct entry of physical results.

5. Standardized Software: Word, Excel. 


\section{High Energy Physics (Cost Center 137)}

\begin{tabular}{|ccccc|}
\hline \multicolumn{4}{c|}{ Primary Contacts } \\
Contact Person & Subject & Fax\# & Phone & E-Mail Address \\
Jim Schlereth & All & N/A & 6281 & jls@hep.anl.gov \\
\hline
\end{tabular}

\section{Personal Computers and Workstations}

Manufacturer

IBM (PC, AT, XT, PS/2)

Apple (II, Macintosh)

Compaq

VAXstation 3000

DECstation

\section{Existing \\ FY1991}

N/A

N/A

N/A

3
Existing

FY1992

N/A

$N / A$

N/A

0

\section{Estimated Total FY1993}

33

20

4

4

\section{Word Processors}

$\begin{array}{cccc}\text { Manufacturer } & \begin{array}{c}\text { Existing } \\ \text { FY1991 }\end{array} & \begin{array}{c}\text { Existing } \\ \text { FY1992 }\end{array} & \begin{array}{c}\text { Estimated Total } \\ \text { FY } 1993\end{array} \\ \text { Exxon/Nydec } & \text { N/A } & \text { N/A } & 6\end{array}$

Software*

CAE/CAD

AutoCAD

Altera

(8)

(1)

(2)
Communication/Terminal Emulation

VTerm

Kermit

(4)

ORCAD

Tango

Spreadsheet

Multiplan

Lotus 1-2-3

Project Management

MacProject II

Utility

DOS

- The numbers of software packages are estimates. 
Network Connections
Existing
FY1992
Estimated Total
FY1993

Lab-wide Ethernet

TCP/IP

15

DECnet

10

30

Both

7

20

7

Please describe systems andior applications that you plan to implement during the next fiscal year in the areas of $(1)$ scientific work stations, (2) personal computing, (3) local area networking, and (4) office automation. Please also indicate standardized software packages for your division.

1. Scientific Work Stations: None planned.

2. Personal Computing: None planned.

3. Local Area Networking: None planned.

4. Office Automation: None planned.

5. Standardized Software: None indicated. 


\section{Human Resources (Cost Center 410 and 416*)}

\section{Primary Contacts}

Contact Person

Subject

Fax\#

Phone

E-Mail Address

George Lales

All

3014

2987

B35710 AT ANLVM

Personal Computers and Workstations

Manufacturer

$$
\text { Existing }
$$

FY1991

Existing

FY1992

Estimated Total

FY1993

IBM ( PC, AT, XT, PS/2)

45

Macintosh

1

55

59

6

\section{Software}

Communcication/Terminal Emulation

Kermit

IBM PC3270

(1)

Telex

Maxess

Word Processing/Editing

Wond

KEDIT

Word for Windows

Integrated Packages

Windows 3.0

Graphics

Harvard Graphics

(2)
Other

Org. Plus

Cobra

Network Schedules (1 NET)

Omni Page

MacLinc PC

CC: Mail (1 NET)

Form Base

(3)

Spreadsheet

Lotus 1-2-3

Excel

(3)

Database

* Includes Secretarial and Clerical Services 


\section{Network Connections}

$\begin{array}{cc}\text { Existing } & \text { Estimated Total } \\ \text { FY1992 } & \text { FY1993 }\end{array}$

Local 3Com

53

Epson Server (LAN Manager)

53

63 (Macs will be added)

63

Planned activities andlor applications to be implemented during the next fiscal year in the areas of (1) scientific work stations, (2) personal computing, (3) local area networking, and (4) office automation. Please also indicate standardized software packages for your division.

1. Scientific Work Stations: None planned.

2. Personal Computing: Continue to upgrade microsoft products. Up communications with more shared spaces, mail, scheduling. Automate project management tools for managers. Push personal databases.

3. Local Area Networking: Plan to investigate ad-hoc reporting on the server with DBMS SQL or dBase reporting interface. Move towards client server technology. Finish migration to LAN-Manager. Incorporate Macintoshers. Continue with development of multi-user Clipper information systems.

4. Office Automation: Continue to pursue an acceptable forms processing system on the LAN.

5. Standardized Software: Microsoft Word for Window:s; Excel; Windows; Harvard Graphics; CC: Mail; Network Scheduler. 


\section{Inspections (Cost Center 336)}

\section{Primary Contacts}

Contact Person

Subject

Fax\#

Phone

E-Mail Address

Thomas Busse

Personal Computers

N/A

5713

N/A

\section{Personal Computers and Workstations}

$\begin{array}{lccr}\text { Manufacturer } & \begin{array}{c}\text { Existing } \\ \text { FY1991 }\end{array} & \begin{array}{c}\text { Existing } \\ \text { FY1992 }\end{array} & \begin{array}{r}\text { Estimated } \\ \text { FY199 }\end{array} \\ \text { IBM (PC, AT, XT, PS/2) } & 3 & 3 & 4 \\ \text { Gateway } & 0 & 0 & 2\end{array}$

\section{Software}

Word Processing/Editing

Word

Spreadsheet

Multiplan
(3)

(3)
Database

DBase IV

Please describe systems and/or applications that you plan to implement during the next fiscal year in the areas of $(1)$ scientific work stations, (2) personal computing, (3) local area networking, and (4) office automation. Please also indicate standardized software packages for your division.

1. Scientific Work Stations: None planned.

2. Personal Computing: None planned.

3. Local Area Networking: None planned.

4. Office Automation: None planned.

5. Standardized Software: None indicated. 


\section{Intense Pulsed Neutron Source Program (Cost Center 136)}

\begin{tabular}{|ccccc|} 
& \multicolumn{4}{c|}{ Primary Contacts } \\
Contact Person & Subject & Fax & Phone & E-Mail Address \\
Ira Bresof & All & N/A & 8705 & BRESOF AT ANLPNS \\
\hline
\end{tabular}

Personal Computers and Workstations

Manufacturer

IBM (PC, AT, XT, PS/2)

Apple (II, Macintosh)

Compaq

Gateway

Epson

Tri Star
Existing
FY1991

11
19
7
0
0
0

Existing
FY1992

6

26

6

6

2
Estimated Total FY1993

6
29
6
10
2
1

\section{Software}

Word Processing/Editing MacWrite

Word

Text Editor

Mass I1

WordPerfect

Darabare

dBASE III Plus

Mass11-Manage

dBASE II Mac

DEC RDB

Integrated Packages

Excel

\section{Utility}

Misc. MacIntosh
(1)

(I)
Graphics

MacDraw II

MacPaint

Mass11 Draw

KaleidaGraph

DeltaGraph

(5)

(2)

(1)

(8)

(1)

Communication/Terminal Emulation

$\begin{array}{lr}\text { Kermit } & (8) \\ \text { PCSA } & (9) \\ \text { Versaterm Pro } & (14)\end{array}$

Spreadsheet

Multiplan

Quaturo

(3)

(6)

CAE/CAD

Claris CAD

(2) 


\section{Network Connections \\ Existing \\ FY1992 \\ Estimated Total \\ FY1993}

Lab-wide Ethernet

DECnet

Lab-wide AppleTalk

1

2

1

2

Please describe systems and/or applications that you plan to implement during the next fiscal year in the areas of (I) scientific work stations, (2) personal computing, (3) local area networking, and (4) office automation. Please also indicate standardized software packages for your division.

1. Scientific Work Stations: We plan to add two UNIX Workstations and two VMS Workstations in FY1993.

2. Personal Computing: We plan to add two Macintosh computers and two 486 PCs.

3. Local Area Networking: We will continue to use ethernet hardware. Communication software will be a mixture of Internet and DECNET.

4. Office Automation: We have standardized on Microsoft Word on the Macintosh and are considering changing from Mass 11 to Microsoft Word or WordPerfect on the PC.

5. Standardized Software: Spreadhsheet:

Microsoft - EXCEL

Communications:

Word Processing:

Macintosh - Versaterm-Pro, PC- Kermit

Mail:

Macintosh - Microsoft Word

Macintosh - Quick Mail, PC - Pathworks 


\section{Lodging Facilites (Cost Center 222)}

\begin{tabular}{|ccccc|}
\hline \multicolumn{4}{c|}{ Primary Contacts } \\
Contact Person & Subject & Fax\# & Phone & E-Mail Address \\
Pat Carson & All & N/A & 2580 & N/A \\
\hline
\end{tabular}

\begin{tabular}{|cccc|}
\hline & Personal Computers and Workstations & \\
& Existing & Existing & Estimated Total \\
Manufacturer & FY1991 & FY1992 & FY1993 \\
Mitac (PC) & 2 & 3 & 3 \\
\hline
\end{tabular}

\section{Software}

Word Processing/Editing

WordPerfect

Spreadsheat

Multiplan

Lotus 1-2-3

(1)

(1)
Database

dBASE IV

Hotel System
(2)

(1)

Please describe systems andlor applications that you plan to implement during the next fiscal year in the areas of (1) scientific work stations, (2) personal computing. (3) local area networking, and (4) office automation. Please also indicate standardized software packages for your division.

1. Scientific Work Stations: None planned.

2. Personal Computing: Continued expansion of personal computing capabilities is planned for the next year.

3. Local Area Networking: Local area network installation in Buildings $201,4,810,827$ is planned for next year. Expansion of 214 network is planned for the last half of 1992.

4. Office Automation: None planned.

5. Standardized Software: A committee to select and standardize software and hardware system for PFS has been formed. John Mitchell is chairperson, $\times 8610$. The charter of this committee is to maintain consistent computing activities with the PFS Division. 
Materials Science Division (Cost Center 105)

Primary Contacts

$\begin{array}{ccccc}\text { Contact Person } & \text { Subject } & \text { Fax\# } & \text { Phone } & \text { E-Mail Address } \\ \text { Paul Domagala } & \text { All } & 4798 & 5197 & \text { domagala@anl.gov }\end{array}$

\section{Personal Computers and Workstations}

Manufacturer

IBM (PC, AT, XT, PS/2)

Apple (II, Macintosh)

Compaq

Zenith

NEC

SUN

Silicon Graphics

VAX

Next

\section{Existing \\ FY1991}

19

125

8

0

0

3

2

6

5
Existing
FY1992

20
157
8
2
2
3
2
7
5

\section{Estimated Total} FY1993

24
180
8
2
2
3
2
7
5

\section{Software*}
Graphics
MacDraw II
MacPaint
Stateview
MacDraw
MacDraft
CricketGraph
Languages/Compilers
LS Fortran
MacFortran
MPW Pascal
LS Pascal
LS C
Word Processing/Editing MacWrite
Wond
Integrated Packages
Excel
Security
Nightwatch

- Software totals are estimates.

(2)
Other

MiniCad

QuickMail

Reflections

Timbuktu

Mathematica

Dreams

Dicer

MathType

Graceland Responder

(1)

(90)

(1)

(47)

(5)

(3)

(157)

Communication/Terminal Emulation

VersaTerm Pro

(30)

Versa Term

NCSA Telnet

Database

dBASE III Plus

Filemaker II

Utilities

Disk Express

(2)

SUM 


$\begin{array}{cc}\text { Network Connections (nodes) } & \\ & \\ \text { Existing } & \text { Estimated Total } \\ \text { FY1992 } & \text { FY1993 } \\ 119 & 135 \\ 143 & 175\end{array}$

Lab-wide Ethernet

Please describe systems and/or applications that you plan to implement during the next fiscal year in the areas of (1) scientific work stations, (2) personal computing. (3) local area networking, and (4) office automation. Please also indicate standardized software packages for your division.

1. Scientific Work Stations: The three existing Sun Sparc IIs will be upgraded to Sparc 10s

2. Personal Computing: A remote tape backup system for the Macintosh computers on the Appletalk network will be added.

3. Local Area Networking: Building 223 network will be rewired, providing ethemet (thin) access to all offices.

4. Office Automation: One of two flat-bed scanners will be added.

5. Standandized Software: Standard Macintosh software now includes Word, Quickmail, Graceland Responder 


\section{Materials and Components Technology (Cost Center 114)}

\begin{tabular}{|ccccc|}
\hline \multicolumn{4}{c|}{ Primary Contacts } \\
Contact Person & Subject & Fax\# & Phone & E-Mail Address \\
Bill Shack & Macintosh & 4798 & 5137 & wj_shack@qmgate.anl.gov \\
\hline
\end{tabular}

Personal Computers and Workstations

$\begin{array}{lccc} & \text { Existing } & \text { Existing } & \text { Estimated Total } \\ \text { Manufacturer } & \text { FY1991 } & \text { FY1992 } & \\ \text { IBM (PC, AT, XT, PS/2) } & 35 & 37 & 37 \\ \text { Apple (II, Macintosh) } & 55 & 60 & 85 \\ \text { Compaq } & 2 & 2 & 2 \\ \text { Sun \& HP Workstations } & 7 & 10 & 10 \\ \text { Zenith } & 4 & 4 & 4 \\ \text { PC Limited } & 10 & 10 & 10 \\ \text { AST Research } & 1 & 1 & 1 \\ \text { PC Clones } & 7 & 7 & 12\end{array}$

Word Processors

$\begin{array}{cccc}\text { Manufacturer } & \begin{array}{c}\text { Existing } \\ \text { FY1991 }\end{array} & \begin{array}{c}\text { Existing } \\ \text { FY1992 }\end{array} & \begin{array}{c}\text { Estimated Total } \\ \text { FY } 1993\end{array} \\ \text { Exxon/Vydec } & 1 & & 1 \\ \text { NBI } & 2 & 1 & 2\end{array}$

\section{Software*}

Graphics

MacDraw II

MacDraw

MacPaint

CricketDraw

KaleidaGraph

DeltaGraph

\section{Utilities}

Diskfit

Super LaserSpool

Retrospect Remote
Communication/Terminal Emulation

$$
\begin{aligned}
& \text { VTerm } \\
& \text { Kermit } \\
& \text { QuickMail } \\
& \text { Telnet } \\
& \text { Mail Sender }
\end{aligned}
$$

Languages/Compilers

Fortran
Pascal
Basic
C

- The numbers of software packages are estimates. 


\section{Software (continued)*}
Security
SUM
Folder Lock
Disinfectant
Spreadsheet
Lotus
Word Processing/Editing
Word

\section{Database}

dBASE III Plus

Filemaker Pro

\section{CAE/CAD}

MiniCad

(2)

Dreans

Integrated Packages

Excel

- The numbers of software packages are estimates.

\section{Network Connections \\ Existing \\ FY1992 \\ Estimated Total \\ FY1993}

Lab-wide Ethernet

TCP/IP

Lab-wide AppleTalk

(Fax Modem for E-mail Network)
18 Nodes +3 Gator Boxes

60 Nodes
25 Nodes +3 Gator Boxes 85

Please describe systems andlor applications that you plan to implement during the next fiscal year in the areas of (1) scientific work stations, (2) personal computing. (3) local area networking, and (4) office automation. Please also indicate standardized software packages for your division.

1. Scientific Work Stations: Will probably add $2-3$ workstations (SUN or HP).

2. Personal Computing: Will continue to add Macintoshes for general use. Gateway clones for Laboratory data acquisition.

3. Local Area Networking: Will attach to new hubs to provide 10Mb Ethernet connections between buildings.

4. Office Automation: None planned.

5. Standardized Software: Word, Excel, FileMaker Pro, KaleidaGraph or DeltaGraph Professional. 
Materials and Services Department (Cost Center 315)

\begin{tabular}{|llccc|}
\hline \multicolumn{4}{c|}{ Primary Contacts } \\
Consact Person & Subject & Fax\# & Phone & E-Mail Address \\
Charles Robinson & Inventory & 3197 & 2928 & N/A \\
\hline
\end{tabular}

Personal Computers and Workstations

$\begin{array}{cccc} & \text { Existing } & \text { Existing } & \text { Estimated Total } \\ \text { Manufacturer } & \text { FY1991 } & \text { FY1992 } & \text { FY1993 } \\ \text { IBM (PC, AT, XT, PS/2) } & 24 & 21 & 19 \\ \text { Gateway } & 4 & 13 & 17\end{array}$

\section{Software}

Word Processing

Wand

KEDIT

Multimate

WordPerfect 5.1 (Network)

Right Writer

Communication/Terminal Emulation

Kermit

Relay Gold

Reflection

Crosstalk

Spreadsheet

Multiplan

Quattro Pro (Network)

Integrated Packages

Excel

\section{Desktop Publishing}

Xerox Venura 3.0
(8)

(1)

(6)

(7)

(9)

(5)

(2)

(1)
Other

Hazmat Doc

Sideways

Regscan 49

Report Writer

Word Link

Genifer

Xerox Formbase

Windows 3.0

Windows 3.1

(1)

(1)

(1)

(1)

(1)

Database

dBASE III Plus

dBASE IV

FoxBase 2.1

(7)

(1)

(1)

Graphics

Chart

Utility

Norton Utilities 
Please describe systems andlor applications that you plan to implement during the next fiscal year in the areas of (1) scientific work stations, (2) personal computing. (3) local area networking, and (4) office automation. Please also indicate standardized software packages for your division.

1. Scientific Work Stations: None planned.

2. Personal Computing: Personal productivity tool utilized such as word processing, database, desktop publishing, and form and database generations.

3. Local Area Networking: Novell 3.11

4. Office Automation: Bar coding

5. Standardized Software: None indicated. 


\section{Mathematics and Computer Science (Cost Center 145)}

\begin{tabular}{|ccccc|}
\hline \multicolumn{4}{c|}{ Primary Contacts } \\
Contaci Person & Subject & Fax & Phone & E-Mail Address \\
Gene Rackow & All & & 7126 & rackow@mcs.anl.gov \\
\hline
\end{tabular}

Personal Computers and Workstations

Manufacturer

IBM (PC, AT, XT, PS/2)

Apple (II, Macintosh)

Sun

Zenith

NeXT

SGI

IBM (RS/6000)

$\begin{array}{ll}\text { Existing } & \text { Existing } \\ \text { FY1991 } & \text { FY1992 }\end{array}$

6
1
58
2
20

FY1992

6
1
70
2
20

\section{Estimated Total} FY1993

6
1
70
2
40
5
8

\section{Software}

Word Processers/Editing
MacWrite
TeX
LaTeX
Languages/Compilers
C
C++
Prolog
Fortran

(1)
Graphics
MacDraw II
MacPaint
AVS

(1)

(1)

(5)

Spreadsheet

Multiplan

(1)

Communication/Terminal Emulation

VTerm

(6)

\section{Network Connections}

Existing

FY1992

\section{Estimated Total \\ FY1993}

Lab-wide Ethernet

TCP/IP

all machines

all machines 
Planned activities and/or applications to be implemented during the next fiscal year in the areas of (1) scientific work stations, (2) personal computing, (3) local area networking, and (4) office automation. Please also indicate standardized software packaages for your division.

1. Scientific Work Stations: None planned.

2. Personal Computing: None planned.

3. Local Area Networking: None planned.

4. Office Automation: None planned.

5. Standardized Software: None indicated. 
Media Services Department (Cost Center 260)

\begin{tabular}{|ccccc|}
\hline \multicolumn{5}{c|}{ Primary Contacts } \\
Contact Person & Subject & Fax\# & Phone & E-Mail Address \\
Lee Wagar & All & 5974 & 5603 & b20429@anlvm.ctd.anl.gov \\
\hline
\end{tabular}

Personal Computers and Workstations

Manufacturer

Existing

Existing

FY1991

FY1992

Estimated Total FY1993

IBM (PC, AT, XT, PS/2)

Apple (Macintosh)

20

13

17

20

14

22

\section{Software}

Word Processing/Editing

WordPerfect

Word

(2 Mac, 8 PC)

(13 Mac, 7 PC)

Spreadsheet

Quattro Pro

Excel

Presentation Visuals

Persuasion

Database

DBase III Plus

FileMaker Pro
(6 PC)

(3 Mac)

(4 Mac)

(11 PC)

(3 Mac)
Graphics

MacDraw II

Canvas

Freehand

Claris CAD

DeltaGraph

KaleidaGraph

Illustrator

MacFlow

\section{Desktop Publishing}

PageMaker

QuarkXpress

(11 Mac)

(1 Mac)

Communication/Terminal Emulation

Kermit

NCSA Telnet

(N/A Mac, PC)

(N/A Mac)

Equations

Expressionist

MathType
$(6 \mathrm{Mac})$

(1 Mac)
Utility

MacLisk

Norton Utilities

DOS Mounter

Disinfectant

XTreePro Gold

VirusScan
(1 Mac)

(1 PC)

(7 Mac)

(N/A Mac)

(7 PC)

(N/A PC) 


\section{Network Connections}

$\begin{array}{lc}\text { Existing } & \text { Estimated Total } \\ \text { FY1992 } & \text { FY1993 }\end{array}$

Lab-wide Ethernet

TCP/IP Telnet

10

12

XNS (3Com)

20

17

LocalTalk (AppleShare, QuickMail, AlisaTalk)

Local AppleTalk

15

20

15

20

Please describe systems andlor applications that you plan to implement during the next fiscal year in the areas of (I) scientific work stations, (2) personal computing. (3) local area networking, and (4) office automation. Please also indicate standardized software packages for your division.

1. Scientific Work Stations: None planned.

2. Personal Computing: Acquire divisional Macintosh server.

3. Local Area Networking: None planned.

4. Office Automation: None planned.

5. Standardized Software: None indicated. 
Motion Picture Unit (Cost Center 276)

\begin{tabular}{|rrrrr|}
\hline \multicolumn{5}{c|}{ Primary Contacts } \\
Contact Person & Subject & Fax\# & Phone & E-Mail Address \\
Gail Farmer & IBM & 5545 & 7451 & N/A \\
\hline
\end{tabular}

Personal Computers and Workstations

$\begin{array}{lccc} & \begin{array}{c}\text { Existing } \\ \text { Manufacturer }\end{array} & \begin{array}{c}\text { Existing } \\ \text { FY1992 }\end{array} & \begin{array}{c}\text { Estimated Total } \\ \text { FY1993 }\end{array} \\ \text { IBM (PC, AT, XT, PS/2) } & 2 & 2 & 2 \\ \text { Amiga 2000 } & 1 & 2 & 2 \\ \text { MacIntosh } & 2 & 2 & 2 \\ \text { Gateway 2000 } & 0 & 0 & 0\end{array}$

\section{Software}

Word Processing

WordPerfect 5.1

(2)

WordPerfect Mac

(1)

Word

(1)

Other

DigiView

Provideo Plus

VideaScape

Sculpt/Animale 3D

Database

dBASE III Plus

(1)

Filemaker

(1)

Aegis Aninuator

Labels Unlimited

Macromind Director

Authorware

Sundance Systems

(1)

(1)

(1)

(1)

(1)

(1)

(1)

(1)

Spreadsheet

Lotus 1-2-3

(1)

Excel

(1) 
Please describe systems andlor applications that you plan to implement during the next fiscal year in the areas of (1) scientific work stations, (2) personal computing, (3) local area networking, and (4) office automation. Please also indicate standardized software packages for your division.

1. Scientific Work Stations: None planned.

2. Personal Computing: Purchase PC's for staff use.

3. Local Area Networking: None lanned.

4. Office Automation: None planned.

5. Standardized Software: None indicated. 
Office of the Chief Financial Officer (Cost Centers 400, 401, 402, and 403)

\section{Primary Contacts}

Contact Person

Subject

Fax\#

Phone

E-Mail Address

Paul Vignola

All

6397

7589

Vignola@anl.gov

\section{Personal Computers and Workstations}

Manufacturer

IBM (PC, AT, XT, PS/2)

Gateway 2000 Portable

$\begin{array}{cc}\text { Existing } & \text { Existing } \\ \text { FY1991 } & \text { FY1992 } \\ 91 & 100 \\ 3 & 3\end{array}$

Estimated Total FY1993

3

\section{Software}

Word Processing/Editing MS-Word for Windows

Database

DBase IV

Spreadsheet

Lotus 1-2-3

Excel

Quaturo 2.0

Operating System/Environments

MS DOS 5.0

MS Windows 3.1

Communication/Terminal Emulation

Kermit

Maxess by $3 \mathrm{Com}$

Other

RODEPC

Disc 1099PC
Graphics

SuperImage

MS-Power Point

(2)

Desktop Publishing

Byline

Project Management

MS Project

Electronic Mail

CC: Mail

Languages/Compilers

4GL, Jr.

Metaview

MS Visual Basic

Utility

Norton Utilities

Sidekick 


\section{Network Connections}

$\begin{array}{lc}\text { Existing } & \text { Total } \\ \text { FY1992 } & \text { FY1993 }\end{array}$

Local 3Com

LAN Manager

Netware

80

0

$5 \quad 80$

Netware - 0

20

Planned activities and/or applications to be implemented during the next fiscal year in the areas of (I) scientific work stations, (2) personal computing, (3) local area networking, and (4) office automation. Please also indicate standardized software packages for your division.

1. Scientific Work Stations: OCF has acquired an HP Unix Computer on which we will study the feasibility of downsizing the Lab's integrated financial system.

2. Persomal Computing: Our computer stable now houses approximately 110 computers. The first 20 were 8088 class machines. Three are still in service. The second 20 are 80286 machines and will limp along another year. The rest are $803865 x$ 's. This year will be for the 486 .

3. Local Area Networking: Our migration from 3COM's 3+ share network operating system brought us to a hybrid network incorporating MicroSoft's LAN Manager and Novell's Netware.

4. Office Automation: We have chosen MicroSoft's suite of office automation tools including Word for Windows, Excel, PowerPoint, Projech, and Visual Basic.

5. Standardized Software: ANL has Mac's, PC's, NBI's, Unix, and Vax. There exist no ANL software standands. 


\section{Office of the Director (Cost Center 201)}

\begin{tabular}{|ccccc|}
\hline \multicolumn{4}{c|}{ Primary } & Contacts \\
\hline Contact Person & Subject & Fax\# & Phone & E-Mail Address \\
Pat Traub & $\begin{array}{c}\text { Strategic Planning Office } \\
\text { Office of the Director }\end{array}$ & 3679 & 4253 & PKT AT \\
& ANLADM1 \\
\hline
\end{tabular}

\section{Personal Computers and Workstations}

Manufacturer

IBM (PC, AT, XT, PS/2)

Gateway 2000

$$
\begin{aligned}
& \text { Existing } \\
& \text { FY1991 }
\end{aligned}
$$

7

6
Existing

FY1992

7

11
Estimated Total FY1993

7

11

\section{Software}

Word Processing/Editing

Multimate version 4

DBase IV

Word Perfect

Powerpoint for Windows

Grammatic IV

Desktop Publishing

Always

Excel

Other

Flowchar II+

Sidekick

Norton Adv.
(5)

(10)

(4)

(3)

(1)

(4)

(1)

(1)

(1)
Spreadsheet

Multiplan

Lotus 1-2-3

Quaturo

(1)

(3)

(1)

Database

DBase III Plus

(3)

Languages/Compilers

Basic

(1)

Windows 386

Communication/Terminal Emulation

Kermit

(2)

Procomm + 


\section{Network Connections}

$\begin{array}{lc}\text { Existing } & \text { Estimated Total } \\ \text { FY1992 } & \text { FY1993 }\end{array}$

Novell

9

11

Please describe systems andlor applications that you plan to implement during the next fiscal year in the areas of (1) scientific work stations, (2) personal computing, (3) local area networking, and (4) office automation. Please also indicale standardized software packages for your division.

1. Scientific Work Stations: None planned.

2. Personal Computing: None planned.

3. Local Area Networking: Novell

4. Office Automation: None planned

5. Standardized Software: None indicated. 


\section{Office of Public Affairs (Cost Center 275)}

\begin{tabular}{|ccccc|}
\hline \multicolumn{5}{c}{ Primary Contacts } \\
Contact Person & Subject & Fax\# & Phone & E-Mail Address \\
Marilyn Wittkofski & All & 5274 & 5579 & WRM AT ANLADM1 \\
\hline
\end{tabular}

\section{Personal Computers and Workstations}

$\begin{array}{lccc}\text { Manufacturer } & \begin{array}{c}\text { Existing } \\ \text { FY1991 }\end{array} & \begin{array}{c}\text { Existing } \\ \text { FY1992 }\end{array} & \begin{array}{c}\text { Estimated Total } \\ \text { FY1993 }\end{array} \\ \text { Apple (II, Macintosh) } & 15 & 15 & 20 \\ \text { IBM (PC, AT, XT, PS/2) } & 1 & 1 & 1 \\ \text { Vydec 1800 } & 1 & 1 & 1\end{array}$

\section{Word Processors}

$\begin{array}{cccc}\text { Manufacturer } & \begin{array}{c}\text { Existing } \\ \text { FY1991 }\end{array} & \begin{array}{c}\text { Existing } \\ \text { FY1992 }\end{array} & \begin{array}{c}\text { Estimated Total } \\ \text { FY 1993 }\end{array} \\ \text { NBI } & 8 & 8 & 8\end{array}$

\section{Software}

Word Processing/Editing Microsoft Word

FileMaker

HyperCard

MultiFinder

Desktop Publishing

PageMaker
(20)

(10)

(10)

(10)

(6)
Graphics

MacDraw II

(10)

MacPaint

(10)

Integrated Packages

Excel

Communication/Terminal Emulation Kermit 


\section{Network Connections}

$\begin{array}{cc}\text { Existing } & \text { Estimated Total } \\ \text { FY1992 } & \text { FY1993 }\end{array}$

Local AppleTalk

Please describe systems and lor applications that you plan to implement during tyhe next fiscal year in the areas of (1) scientific work stations, (2) personal computing. (3) local area networking, and (4) office automation. Please also indicale standardized software packages for your division.

1. Scientific Work Stations: None planned.

2. Personal Computing: None planned.

3. Local Area Networking: None planned.

4. Office Automation: None planned.

5. Standardized Software: Normal upgrade of existing software. 


\section{Physical Research (Cost Center 273)}

\section{Primary Contacts}

Contact Person

Subject

Fax\#

Phone

E-Mail Address

Emest VanBerkum

All

6720

3750

B07412 AT ANLVM

\section{Personal Computers and Workstations}

Manufacturer

Apple (II, Macintosh)

Gateway 2000
Existing

FY1991

3

0

\section{Existing}

FY1992

4
Estimated Total

FY1993

\section{Word Processors}

Manufacturer

NBI
Existing

FY1991

0
Existing

FY1992

0
4

\section{Software}

Word Processing/Editing MacWrite

Word
(4)

(4)
Graphics

MacDraw II
MacPaint
SuperPaint
MacDraft

Network Connections

Existing

FY1992
Estimated Total

FY1993

Local AppleTalk

4

4 
Please describe systems wid/or applications that you plan to implement during the next fiscal year in the areas of (1) scientific work stations, personal computing, (3) local area networking, and (4) office automation. Please also indicale slandardized sy fware packages for your division.

1. Scientific Work Stations: None planned.

2. Personal Computing: Continue use of PCs for word processing, spreadsheets, graphics, electronic mail, and link to ORPS system.

3. Local Area Networking: Macintosh Apple talk network with Quick Mail.

4. Office Automation: None planned.

5. Standardized Software: None identified. 


\section{Plant Facilities and Services--Plant Manager's Office (Cost Center 506)}

\section{Primary Contacts}

$\begin{array}{ccccc}\text { Contact Person } & \text { Subject } & \text { Fax\# } & \text { Phone } & \text { E-Mail Address } \\ \text { John Mitchell } & \text { All } & \text { N/A } & 8610 & \text { N/A } \\ \text { Susanne Prodehl Forst } & \text { All } & \text { N/A } & 2922 & \text { N/A }\end{array}$

Personal Computers and Workstations

$\begin{array}{cccc} & \text { Existing } & \text { Existing } & \text { Estimated Total } \\ \text { Manufacturer } & \text { FY1991 } & \text { FY1992 } & \text { FY1993 }\end{array}$

Gateway 2000

2

9

9

\section{Word Processors}

$\begin{array}{cccc}\text { Manufacturer } & \text { Existing } & \text { Existing } & \text { Estimated Total } \\ \text { FYI } 531 & \text { FY1992 } & \text { FY } 1993 \\ \text { NBI } & 5 & 4 & 0\end{array}$

\section{Software}

Word Processing

WordPerfect

Communication/Terminal Emulation

Procomm

Kermit

(3)

(3)

MS Developers Kit

Multi-tasking

Enable

(1)
Spreadsheet

Multiplan

MS Excel

(1)

(6)

Database

DBase III Plus
DBase IV
SuperCalc

(1)

(6)

(1)

Project Management

MS Project

(2)

Desktop Publishing 


\section{Software (continued)}

\section{Scanning \\ Imagin \\ Color Lab \\ Operating Systems \\ Windows 3.1 \\ WP Office}

DOE

PFMD

CAS

Tiger Team Database (ESHQA)
(1)
Utility

Calendar Creator

PC Tools

Name Disk

(1)

(1)

(1)

Graphics/Presentation

Corel Draw

Draw Penf

Powerpoint

Artline

Presentation T

Perform

PC Point Brush IV
(1)

(1)

Please describe systems and/or applications that you plan to implement during the next fiscal year in the areas of (1) scientific work stations, (2) personal computing. (3) local area networking, and (4) office automation. Please also indicase standardized software packages for your division.

1. Scientific Work Stations: None planned.

2. Personal Computing: Continued expansion of personal computing capabilities is planned for the next year.

3. Local Area Networking: Local area network installation in Buildings $201,4,810,827$ is planned for next year. Expansion of 214 network is planned for the last half of 1992.

4. Office Automation: None planned.

5. Standardized Software: A committee to select and standardize software and hardware system for PFS has been formed. John Mitchell is chairperson, $\mathbf{x 8 6 1 0}$. The charter of this committee is to maintain consistent computing activities with the PFS Division. 


\section{Procurement Department (Cost Center 322)}

\begin{tabular}{|ccccc|}
\hline \multicolumn{4}{c|}{ Primary Contacts } \\
Contact Person & Subject & Fax\# & Phone & E-Mail Address \\
Norman Goetz & All & 4517 & 7028 & N/A \\
\hline
\end{tabular}

\begin{tabular}{|cccc|}
\hline & \multicolumn{1}{c|}{ Personal Computers and Workstations } \\
& Existing & Existing & Estimated Totol \\
Manufacturer & FYI991 & FY1992 & FY1993 \\
IBM & 39 & 35 & 35 \\
Apple & 1 & 1 & 1 \\
Galeway & 1 & 22 & 22 \\
\hline
\end{tabular}

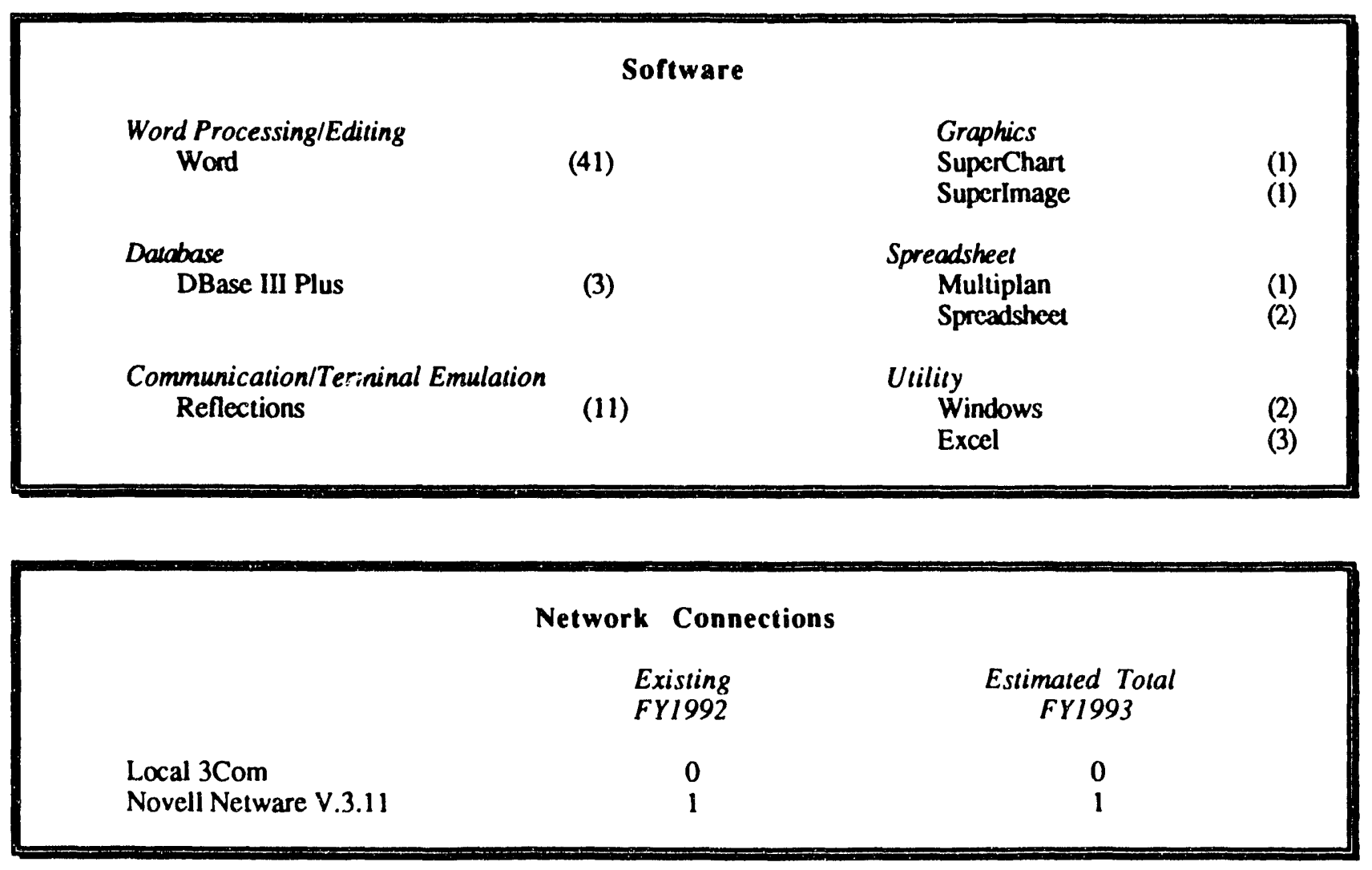




\section{Network Connections}

Existing

FY1992

\section{Estimated Total}

FY1993
Local 3Com

Novel Netware v 3.11
1
0

Please describe systems and/or applications that you plan to implement during the next fiscal year in the areas of $(1)$ scientific work stations, (2) personal computing. (3) local area networking, and (4) office automation. Please also indicate standardized software packages for your division.

1. Scientific Work Stations: None planned.

2. Personal Computing: Personal Productivity tools will be used, such as word processing, database, and spreadsheet to facilitate administrative and magerial functions.

3. Local Area Networking: Plan to replace current hardware due to continued hardware failure and to meet the needs of current procurement databases being rewritten/updated. Currently upgrading network operating system from 3Com 3+Share to Novel Netware vV3.11.

4. Office Automation: Current local area network houses office productivity application software )databases, word processors) allowing the sharing of peripherals such as hard drives and printers.

5. Standardized Software: None indicated. 


\section{Reactor Analysis (Cost Center 116)}

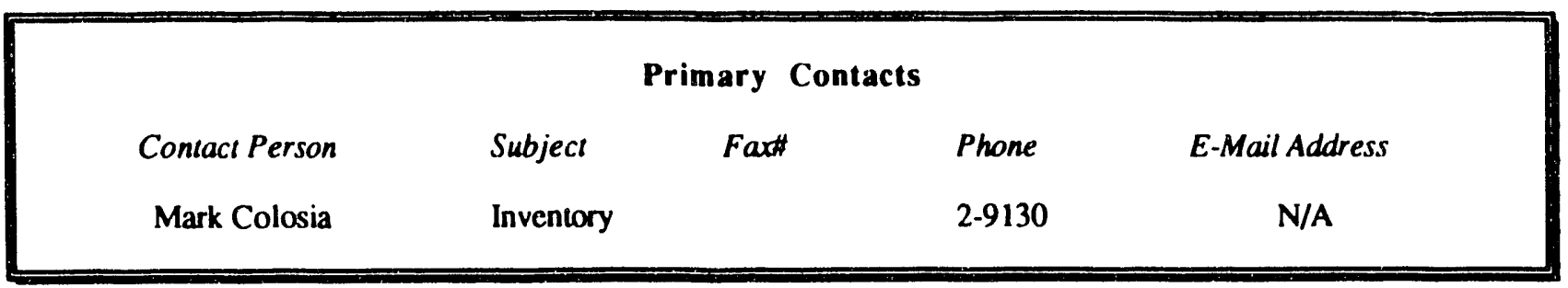

\begin{tabular}{|c|c|c|c|}
\hline \multicolumn{4}{|c|}{ Personal Computers and Workstations } \\
\hline Manufacturer & $\begin{array}{l}\text { Existing } \\
\text { FY1991 }\end{array}$ & $\begin{array}{l}\text { Existing } \\
\text { FYl992 }\end{array}$ & $\begin{array}{c}\text { Estimated Tolal } \\
\text { FY } 1993\end{array}$ \\
\hline IBM (PC, AT, XT, PS/2) & 11 & 11 & 11 \\
\hline Apple (II, Macintosh) & 1 & 2 & 2 \\
\hline Compaq & 1 & 0 & 0 \\
\hline Microstar & 3 & $\mathbf{0}$ & $\mathbf{0}$ \\
\hline Digital & 1 & 0 & 0 \\
\hline Galeway & 0 & 10 & 12 \\
\hline Archic & $\mathbf{0}$ & 1 & 1 \\
\hline dik & 0 & 1 & 1 \\
\hline Kinetic & 0 & 1 & 1 \\
\hline LSI & 0 & 1 & 1 \\
\hline Zenith & 0 & 1 & 1 \\
\hline Sun & 2 & 18 & 18 \\
\hline IBM RS6000 & 0 & 2 & 2 \\
\hline
\end{tabular}

\section{Word Processors}

Manufacturer

NBI
Existing

FY1991

0
Existing

FY1992

0
Estimated Total FY1993

\section{Software on PC's}

Communications/Terminal Emulation

Crosstalk

Kermit

Laplink

Lifeline

MacLink Plus 5.02

NCSA

PC-NFS

WIS
(1)
Database

Fox Base +

Foxpro 2.01

Paradox

Rapid File

Spreadsheet

Lotus 1-2-3

Microsoft Excel

Quaturo Pro
(1)

(1)

(1)

(1)

(9)

(6)

(6) 
Graphics

Cricket Graph

(1)

Delta Graph

(1)

Dissila

DrawPerfect 1.1

(2)

(1)

Generic CADD 5.0

Grapher

GSI Drivers

(DISSPLA 5.00)

MacDraw II

Microsoft Mouse and

Paintbrush

Microsoft Powerprint

Tech-Graph-Pad

Operating System

DOS

Microsoft MS-DOS

Languages

Microsoft Basic

Microsoft Fortran 4.1

(1)

Microsoft Macro

Assembler 5.1

Microsoft Quick Basic

Microsoft Quick C

QBasic

Ryan-McFarland Fortran

Turbo C 2.0 with

Assembler and Debugger

Turbo Pascal

(1)

\section{Utility}

Data Physician Plus

Dequiew

FFM

Filemaker

Filemaker Prs

First Class Hypertext

Framework II

German Tutor

Light

MacroMaker

Mathematica

Minitab (licensed to W.

Ragland through $U$ of $C$ )

Norton Commander

Norton Utilities

PK Zip

Printmaster

Q-DOS

QEMM

Sideways

Stat

StatView II

Supertext

Vaccine

(8)

(1)

(i)

(1)

(1)

(2)

(2)

(1)

(1)

(1)

(1)

Word Processing/Editing

Correct Grammar

KEDIT

MacWrite 4.6

Microsoft Equation Editor

Microsoft Word 5

PC Write

WordPerfect 5.1

WordStar

Software on Workstations

Database

Oracle

(3)

Disspla

Xfig, tgif, ghostview, ghostscript ( $x$-windows contributed - lients)

Sun OS 4.1.1

$\mathrm{X}$-windows. Release 5

Sun OS 3.5
Spreadsheet

xspread ( $x$-windows contributed client)

Languages

Sun Fortran

GNU C, C++ (Free Soft-

ware Foundation)

Sun C

Sun Pascal 


\section{Software on Workstations (continued)}

Word Processing/Editing

WordPerfect 5.0 (10 floating licenses)

Framemaker (4 floating licenses)

GNU emacs (Free Software

Foundation)
Other

Mathematica

Matlab

SAS

Data Views

(14)

Local AppleTalk

TOPS

TCP/IP

\section{Network Connections}

\section{Existing}

FY1992

2

0

30
Estimated Total

FY1993

Planned activities andlor applications to be implemented dwring the next fiscal year in the areas of (1) scientific work stations, (2) personal computing. (3) local area networking, and (4) office ausomation. Please also indicase standardized software packages for your division.

1. Scientific Work Stations: Increase memory and disk space on existing work stations.

2. Personal Computing: None planned.

3. Local Area Networking: Implement AFS client.

4. Office Automation: Add network connections.

5. Standardized Software: None planned. 


\section{Reactor Engineering (Cost Center 112)}

\section{Primary Contacts}

Consact Person

Subject

Fax\#

Phone

E-Mail Address

Jerry Gaston

Inventory

4780

4563

N/A

Personal Computers and Workstations

Manufacturer

IBM (PC, AT, XT, PS/2)

Apple (II, Macintosh)

Compaq

Sun

AST Research

Leading Edge

Standard Brands

Hewleu Packard

\section{Existing}

21

15

9

3

2

6

32
Existing

FY1992

23
17
9
3
2
6
41
1

Estimated Total FY1993

23
19
9
7
2
6
50
1

\section{Software}

Word Processing/Editing

Word

WordPerfect

WordStar

Database

DBase III Plus

FoxPro

Project Management

MicroPlanner

Timeline

Utility

Norton Utilities

CAD
(2)

(1)

(8)

(1)
Graphics

Cricket Graph

Cricket Draw

(2)

(2)

Spreadsheet

Lotus 1-2-3

Quattro Pro

Excel

Languages/Compilers

Pascal

(8)

Fortran

Basic

CAE/CAD

MathCad

Generic CADD 


\section{Network Connections}

$\begin{array}{cc}\text { Existing } & \text { Estimated Total } \\ \text { FY1992 } & \text { FY1993 }\end{array}$

Lab-wide Ethernet

TCP/AP

50

60

DECnet

Please describe systems andlor applications that you plan to implement during tise next fiscal year in the areas of (1) scientific work stations, (2) personal computing, (3) local area networking, and (4) office automation. Please also indicate standardized software packages for your division.

1. Scientific Work Stations: None planned.

2. Personal Computing: None planned.

3. Local Area Networking: None planned.

4. Office Automation: None planned.

5. Standardized Software: None indicated. 


\section{Secretarial and Clerical Services (Cost Center 416)}

See Human Resources (Cost Center 410). Secretarial and Clerical Services and Human Resources share the same word processors, personal computers, workstations, and associated software; however, for budget purposes the cost centers are listed individually. 


\section{Security (Cost Center 232)}

\begin{tabular}{|ccccc|}
\hline \multicolumn{4}{c|}{ Primary Contacts } \\
Contact Person & Subject & Fax\# & Phone & E-Mail Address \\
Rish Ramos & Inventory & 6102 & 5739 & N/A \\
\hline
\end{tabular}

\begin{tabular}{|cccc|}
\hline & Personal Computers & and & \\
& & & \\
Manufacturer & Existing & Existing & Estimated Total \\
IBM & FYI991 & FYI992 & FYI993 \\
AT\&T & 12 & 13 & 13 \\
Mitac & 2 & 2 & 2 \\
Gateway & 6 & 6 & 6 \\
Kinspao & 2 & 2 & 2 \\
& 1 & 1 & 1 \\
\hline
\end{tabular}

\begin{tabular}{|c|c|c|c|}
\hline \multicolumn{4}{|c|}{ Software } \\
\hline $\begin{array}{l}\text { Word Processing/Editing } \\
\text { Multimate Advantage II } \\
\text { WordPerfect }\end{array}$ & $\begin{array}{l}\text { (11) } \\
\text { (14) }\end{array}$ & $\begin{array}{l}\text { Labase } \\
\text { DBase IV } \\
\text { DBase III Plus } \\
\text { FoxPro }\end{array}$ & $\begin{array}{l}\text { (6) } \\
\text { (1) } \\
\text { (1) }\end{array}$ \\
\hline $\begin{array}{l}\text { Spreadsheet } \\
\text { Multiplan }\end{array}$ & (1) & $\begin{array}{l}\text { aphics } \\
\text { Harvard Graphics }\end{array}$ & (3) \\
\hline $\begin{array}{l}\text { Communication } \\
\text { Relay Gold } \\
\text { ProCom }\end{array}$ & $\begin{array}{l}\text { (1) } \\
\text { (1) }\end{array}$ & $\begin{array}{l}\text { Other } \\
\text { Printbar } \\
\text { 3+ Share }\end{array}$ & $\begin{array}{l}(1) \\
\text { (1) }\end{array}$ \\
\hline
\end{tabular}

Network Connections

\section{Existing}

FY1992
Estimated Total FY1993

Local 3Com

1 
Please describe systems and/or applications that you plan to implement during the next fiscal year in the areas of (I) scientific work stations, (2) personal computing. (3) local area networking, and (4) office automation. Please also indicate standardized software packages for your division.

1. Scientific Work Stations: None planned.

2. Personal Computing: None planned.

3. Local Area Networking: None planned.

4. Office Automation: None planned.

5. Standardized Software: None indicated. 


\section{Special Materials (Cost Center 150)}

\begin{tabular}{|ccccc|}
\hline \multicolumn{4}{c|}{ Primary Contacts } \\
Contact Person & Subject & Fax\# & Phone & E-Mail Address \\
Teresa A. Lang & Workstations & 4084 & 6774 & N/A \\
\hline
\end{tabular}

Personal Computers and Workstations

$\begin{array}{lccc} & \text { Existing } & \text { Existing } & \text { Estimated Total } \\ \text { Manufacturer } & \text { FY1991 } & \text { FY1992 } & \text { FY1993 } \\ \text { IBM (PC, AT, XT, PS/2) } & 1 & 1 & 1 \\ \text { PC Limited } & 1 & 1 & 1 \\ \text { AT\&T } & 1 & 1 & 1 \\ \text { AMQ } & 0 & 0 & 0 \\ \text { Transdata } & 0 & 0 & 0\end{array}$

\section{Software}

Word Processing/Editing

Multimate

WordPerfect

Multimate LAN

Utility

Harvard Graphics

Excel
(1)

(3)

(1)

(1)

(4)
Database

DBase III Plus

DBase IV

(1)

(l)

Other

MS DOS

(6)

Star Exchiange

PC DOS

D R DOS
(1)

(1)

\section{Network Connections}

Existing

FY1992

Local 3Com
1
Estimated Total

FY1993 
Please describe systems and/or applications that you plan to implement during the next fiscal year in the areas of $(1)$ scientific work stations, (2) personal computing. (3) local area networking, and (4) office automation. Please also indicate standardized software packages for your division.

1. Scientific Work Stations: None planned.

2. Personal Computing: None planned.

3. Local Area Networking: None planned.

4. Office Automation: None planned.

5. Standardized Software: None indicatid. 


\section{Special Projects Office (Cost Center 197)}

\begin{tabular}{|ccccc|}
\hline \multicolumn{4}{c|}{ Primary Contacts } \\
Contact Person & Subject & Fax\# & Phone & E-Mail Address \\
Debra Petersen & All & N/A & 7561 & N/A \\
\hline
\end{tabular}

Personal Computers and Workstations

Manufacturer

IBM (PC, AT, XT, PS/2)

Compaq

Zenith

Delta Data

Compaq LTE 286

Apple (MacIntosh)

Compaq LTE 386

\section{Existing \\ FY1991}

24

38

1

2

1

3

0

\section{Existing \\ FY1992}

24
38
1
2
1
3
2

\section{Estimated Total}

FY1993

24
38
1
2
1
3
2

\section{Software}

Word Processing/Editing

WordPerfect

WordPerfect LAN

WordPerfect Russian

Bilingual Solutions

Microsoft Word

Grammatik IV

Word Perfect Works

Database

DBase III Plus

DBase IV

DataPerfect

Clipper

PC Album

Q\&A

Spreadsheet

Lotus 1-2-3

Symphony
(11)

(4)

(2)

(3)

(2)
Graphics

GemDraw

Other Gem

DrawPerfect

(16)

(10)

(4)

Desktop Publishing

Ventura

Pagemaker

(2)

Project Management

SuperProject

(4)

Communication/Terminal Emulation

Kermit

Bitcom

Carbon Copy

(6)

(7)

(2)

CAE/CAD

AutoCAD

EasyCAD
(2)

(4) 


\section{Software (continued)}

Integrated Packages

Excel

Framework

(6)

Windows

(1)

(2)

TimeLine

(1)

Other

Think C

(1)

WP Library

(4)

Harvard Project

Utility

Mace

(4)

\section{Network Connections}

Existing

FY1992

Local 3Com

4
Estimated Total FY1993

4

Please describe systems andlor applications that you plan to implement during the next fiscal year in the areas of (1) scientific work stations, (2) personal computing. (3) local area networking, and (4) office automation. Please also indicase standardized software packages for your division.

1. Scientific Work Stations: None planned.

2. Personal Computing: None planned.

3. Local Area Networking: None planned.

4. Office Automation: SPO plans to automate all Procurement/Finance activities.

5. Standardized Software: Word Perfect. 


\section{Support Services Division (Cost Center 216)}

Primary Contacts

$\begin{array}{ccccc}\text { Contact Person } & \text { Subject } & \text { Fax\# } & \text { Phone } & \text { E-Mail Address } \\ \text { Virginia Biondi } & \text { All } & 7740 & 6182 & \text { N/A }\end{array}$

\section{Personal Computers and Workstations}

Manufacturer

IBM (PC, AT, XT, PS/2)

Compaq

Macintosh IICX

Gateway $2000486 / 33$

Toshiba T3200SX

Existing
FY1991
$\mathbf{8}$
1
1
1
0

\section{Existing \\ FY1992}

Estimated Total FY1993

\section{Word Processors}

Manufacturer

NBI

\section{Existing}

FY1991

1
Existing

FY1992

1
Estimated Total FY 1993

9
1
1
2
1

\section{Software}

Word Processing/Editing

Word

WordPerfect

Mac Word

Database

dBASE III Plus

dBASE IV

Foxbase 2.0

Turbo C ++

Borland C ++

Microstep

Magic PC

C-Scape Look \& Feel

C Lib for Btrieve

Burie : for DOS

C-Scape
(8)

(10)

(1)

(6)

(1)

(1)

(1)

(1)

(1)

(1)

(1)

(1)

(1)

(1)
Graphics

Gem Draw

SuperChart

SuperImage

Microsoft Chart

Spreadsheet

Multiplan

Quattro

Lotus 1-2-3

Mac Excel

(1)

(1)

(1)

(2)

(3)

(3)

(1)

(1)

Desktop Publishing

Ventura

(1)

Languages/Compilers

Clipper 


\section{Software (continued)}

Communication/Terminal Emulation

Kermit

Relay Gold

Reflections

Utility

Norton Utilities

PC Tools
(8)

(2)

(1)
Other

ReportWriter

Genifer

Company Ladder

Analyst Gold

PK Zip

(1)

(1)

(1)

(1)

(3)

Project Management

TimeLine

\section{Network Connections}

Existing

FY1992
Estimated Total

FY1993
Local 3Com

Novell Netware V.3.11
0

1
0

Planned activities and/or applications so be implemented during the next fiscal year in the areas of (1) scientific work stations, (2) personal computing, (3) local area networking, and (4) office automation. Please also indicate standardized software packages for your division.

1. Scientific Work Stations: None planned.

2. Personal Computing: Continued PC connection to mainframe via remote dial-in (modems); personal productivity tools utilized such as wordprocessing, databases and utilities, and spreadsheets aiding in administrative and managerial functions.

3. Local Area Networking: Upgraded existing 3Com 3+Share operating system to Novell Netware V 3.11. Replacement of older hardware as needed ( 3 server 3 file server).

4. Office Automation: Continued office productivity application software utilized (databases, spreadsheets, and word processors) and current existence of a local area network sharing hard drive and printers.

5. Standardized Software: None indicated. 


\section{Technical Communications Service Department (Cost Center 265)}

\begin{tabular}{|lllll|}
\hline & \multicolumn{3}{c|}{ Primary Contacts } \\
Contact Person & Subject & Fax \# & Phone & E-Mail Address \\
John Boebinger & Local Talk & 7406 & 6997 & b42252@anl.cv2.ctd.anl.gov \\
& $\begin{array}{l}\text { Network } \\
\text { PC Network }\end{array}$ & & \\
& PCs/Macs & & \\
Jackie Griffith & Word Processing & 3795 & JJD AT ANLEES1 \\
Linda Haley & Graphics & 8820 & N/A \\
\hline
\end{tabular}

\section{Personal Computers and Workstations}

$\begin{array}{cccc} & \text { Existing } & \text { Existing } & \text { Estimated Tolal } \\ \text { Manufacturer } & \text { FY1991 } & \text { FY1992 } & \text { FY1993 }\end{array}$

$\begin{array}{lrrr}\text { IBM (PS/2) } & 5 & 5 & 5 \\ \text { Apple (II, MacIntosh) } & 12 & 3 & 20 \\ \text { Apple Portable Mac } & 2 & 2 & 4 \\ \text { Gateway 2000 } & 19 & 10 & 35 \\ \text { Portable PCs } & 3 & 3 & 8 \\ \text { NBI PC } & 3 & 0 & 3\end{array}$

\section{Word Processors and Network Servers}

$\begin{array}{cccc} & \text { Existing } & \text { Existing } & \text { Estimated Total } \\ \text { Manufacturer } & \text { FY1991 } & \text { FY1992 } & \text { FY 1993 }\end{array}$

$\begin{array}{llll}\text { NBI WP/WS } & 1 & 0 & 1 \\ \text { NBI WP/Server } & 1 & 0 & 1 \\ \text { NBI Unix Server } & 1 & 0 & 1 \\ \text { PC Network Server } & 0 & 2 & 1\end{array}$

\section{Software}

Word Processing/Editing

Word Perfect (DOS)

Word Perfect (Mac)

MS Word (Mac)

MS Word (DOS)

Expressionist

Macwrite
(25)

(5)

(11)

(1)

(4)

(1)
Database

File Maker Pro

Excel

DbaselII

Data Perfect

Plan Perfect

Fast Track Sch.

4
4
3




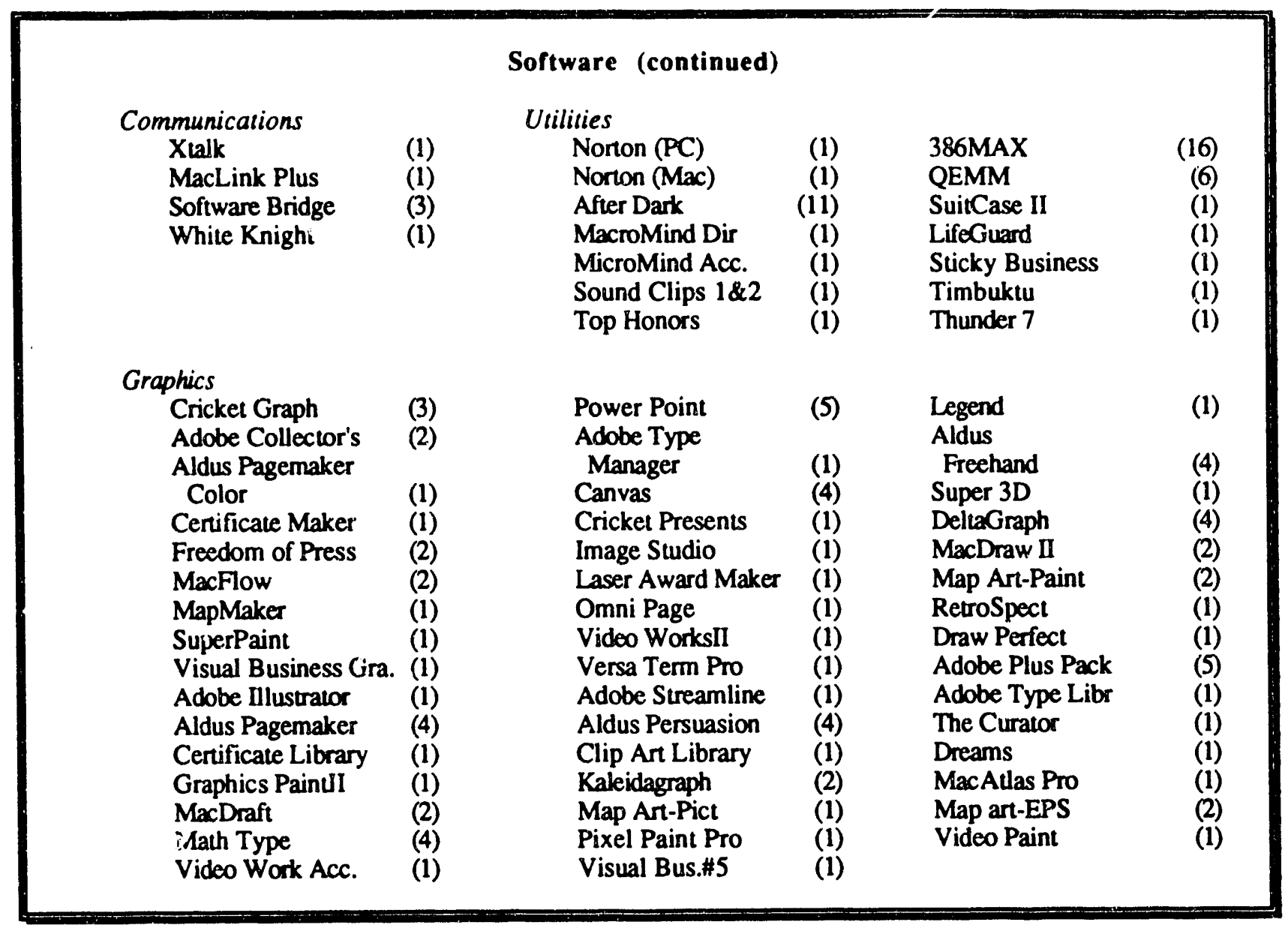

\section{Network Connections}

$$
\begin{aligned}
& \text { Existing } \\
& \text { FY1992 }
\end{aligned}
$$

Lab-wide Ethernet (TCP/IP)

Lab-wide Appletalk
36

18

\section{Estimated Total} FY1993

45 
Please describe systems and/or applications that you plan to implement during the next fiscal year in the areas of (1) scientific work stations, (2) personal computing. (3) local area networking, and (4) office automation. Please also indicate standardized software packages for your division.

1. Scientific Work Stations: None planned.

2. Personal Computing: Continued growth and development of existing applications.

3. Local Area Networking: The network is now Novell-based running CCmail.

4. Office Automation: Both PCs and Macs will coexist, share reousrces, and have common mail capabilities.

5. Standardized Software: None indicated. 


\section{Technical Information Services Department (Cost Center 161)}

\begin{tabular}{|ccccc|}
\hline \multicolumn{4}{c|}{ Primary Contacts } \\
Contact Person & Subject & Fax\# & Phone & E-Mail Address \\
Shannon Savage & All & N/A & 4221 & b27707ti@aim.ctd.anl.gov \\
\hline
\end{tabular}

\begin{tabular}{|lccc|}
\hline \multicolumn{3}{c|}{ Personal Computers and Workstations } \\
& Existing & Existing & Estimated Total \\
& FYI991 & FY1992 & FY1993 \\
Manufacturer & & & 41 \\
IBM (PC, AT, XT, PS/2) & 46 & 41 & 2 \\
Zenith & 2 & 2 & 39 \\
Terminals for AIM* & 31 & 39 & 1 \\
MAC IIc & 1 & 23 & 23 \\
Gateway & 0 & & \\
* Argonne Information Management System & & & \\
\hline
\end{tabular}

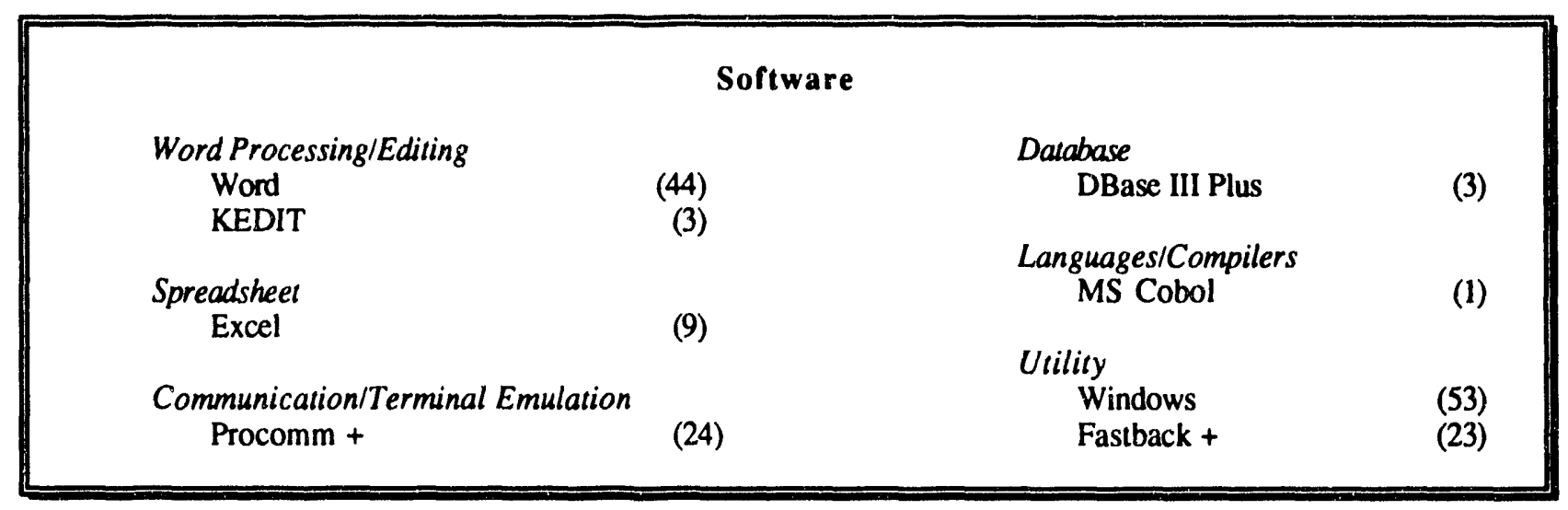


Please describe systems and/or applications that you plan to implement during the next fiscal year in the areas of (1) scientific work stations, (2) personal computing, (3) local area networking, and (4) office automation. Please also indicate standardized software packages for your division.

1. Scientific Work Stations: None planned.

2. Personal Computing: Training for Word for Windows.

3. Local Area Networking: Novell Netware LAN for administrative purposes.

4. Office Automation: None planned.

5. Standardized Software: Word for Windows, Windows 3.1, Procomm for Windows, Excel for Windows. 


\section{Travel Office (Cost Center 319)}

\begin{tabular}{|lcccc|}
\hline & \multicolumn{3}{c|}{ Primary Contacts } & \\
Contact Person & Subject & Fax\# & Phone & E-Mail Address \\
Lawrence McCure & All & 5844 & 4440 & N/A \\
\hline
\end{tabular}

\section{Personal Computers and Workstations}

$\begin{array}{cccc}\text { Manufacturer } & \begin{array}{c}\text { Existing } \\ \text { FY1991 }\end{array} & \begin{array}{c}\text { Existing } \\ \text { FY1992 }\end{array} & \begin{array}{c}\text { Estimated Total } \\ \text { FY1993 }\end{array} \\ \text { IBM (PC, AT, XT, PS/2) } & 2 & 2 & 1 \\ \text { Gateway 2000 386/25 } & 0 & 1 & 1\end{array}$

Software

Word Processing/Editing Word
Database

DBase III Plus

Planned activities andlor applications to be implemented during the next fiscal year in the areas of (1) scientific work stations, (2) personal computing, (3) local area networking, and (4) office automation. Please also indicate standardized software packages for your division.

1. Scientific Work Stations: None planned.

2. Personal Computing: None planned.

3. Local Area Networking: None planned.

4. Office Automation: None planned.

5. Standardized Software: None indicated. 


\section{Utility Systems (Cost Center 510)}

Primary Contacts

$\begin{array}{ccccc}\text { Contact Person } & \text { Subject } & \text { Fax\# } & \text { Phone } & \text { E-Mail Address } \\ \text { Jim Heine } & \text { All } & \text { N/A } & 7922 & \text { N/A }\end{array}$

\section{Personal Computers and Workstations}

$\begin{array}{cccc}\text { Manufacturer } & \begin{array}{c}\text { Existing } \\ \text { FY1991 }\end{array} & \begin{array}{c}\text { Existing } \\ \text { FY1992 }\end{array} & \begin{array}{c}\text { Estimated Total } \\ \text { FY1993 }\end{array} \\ \text { IBM (PC, AT, XT, PS/2) } & 1 & 3 & 3\end{array}$

\section{Software}

Word Processing

MS Word

(2)

(3)

WordPerfect

(1)

(3)

RightWriter

Database

DBase IV

Graphics

DrawPerfect

(2)

Operating Systems

Windows

(3)

Please describe systems andior applications that you plan to implement during the next fiscal year in the areas of $(1)$ scientific work stations, (2) personal computing, (3) local area networking, and (4) office automation. Please also indicate standardized software packages for your division.

1. Scientific Work Stations: None planned.

2. Personal Computing: Continued expansion of personal computing capabilities is planned for the next year.

3. Local Area Networking: Local area network installation in Buildings $201,4,810,827$ is planned for next year. Expansion of 214 network is planned for the last half of 1992.

4. Office Automation: None planned.

5. Standardized Software: A committee to select and standardize software and hardware system for PFS has been formed. John Mitchell is chairperson, $x 8610$. The charter of this committee is to maintain consistent computing activities with the PFS Division. 
Vehicle Maintenance (Cost Centers 316, 317, and 503)

\section{Primary Contacts}

$\begin{array}{lcccc}\text { Contact Person } & \text { Subject } & \text { Fax\# } & \text { Phone } & \text { E-Mail Address } \\ \text { Joanne Jacobson } & \text { All } & \text { N/A } & 7092 & \text { N/A }\end{array}$

Personal Computers and Workstations

$\begin{array}{lccc}\text { Manufacturer } & \begin{array}{c}\text { Existing } \\ \text { FY1991 }\end{array} & \begin{array}{c}\text { Existing } \\ \text { FY1992 }\end{array} & \begin{array}{c}\text { Estimated Total } \\ \text { FY1993 }\end{array} \\ \text { IBM (PC, AT, XT, PS/2) } & 5 & 5 & 5 \\ \text { Gateway 2000 } & 0 & 1 & 1\end{array}$

\section{Software}

Word Processing/Editing

Word

WordPerfect

Spreadsheet

Lotus 1-2-3

Other

49 CFR Regscan

Integrated Packages

MS Windows

MS Works
(1)

(2)

(2)

(1)

(5)

(1)
Database

DBase Illt

(1)

Desktop Publishing

MS Publisher

(1)

Utility

TallGrass Backup

(1)

Communication/Terminal Emulation

Procomm

Kermit

(1)

(1)

\section{Network Connections}

Existing

FY1992
Estimated Tolal

FY1993

DecNet 
Please describe systems and/or applications that you plan to implement during the next fiscal year in the areas of (1) scientific work stations, (2) personal computing, (3) local area networking, and (4) office automation. Please also indicale standardized software packages for your division.

1. Scientific Work Stations: None planned.

2. Personal Computing: Continued expansion of personal computing capabilities is planned for the next year.

3. Local Area Networking: Local area network installation in Buildings 201, 4, 810, 827 is planned for next year. Expansion of 214 network is planned for the last half of 1992.

4. Office Automation: None planned.

5. Standardized Software: A commiuee to select and standardize software and hardware systems for PFS has been formed. John Mitchell is chairperson, $x 8610$. The charter of this committee is to maintain consistent computing activities with the PFS Division. 


\section{Waste Management Operations (Cost Center 505)}

\begin{tabular}{|ccccc|}
\hline \multicolumn{5}{c|}{ Primary Contacts } \\
Contact Person & Subject & Fax\# & Phone & E-Mail Address \\
Duane Bradley & All & & 7307 & B15692A AT ANLVM \\
\hline
\end{tabular}

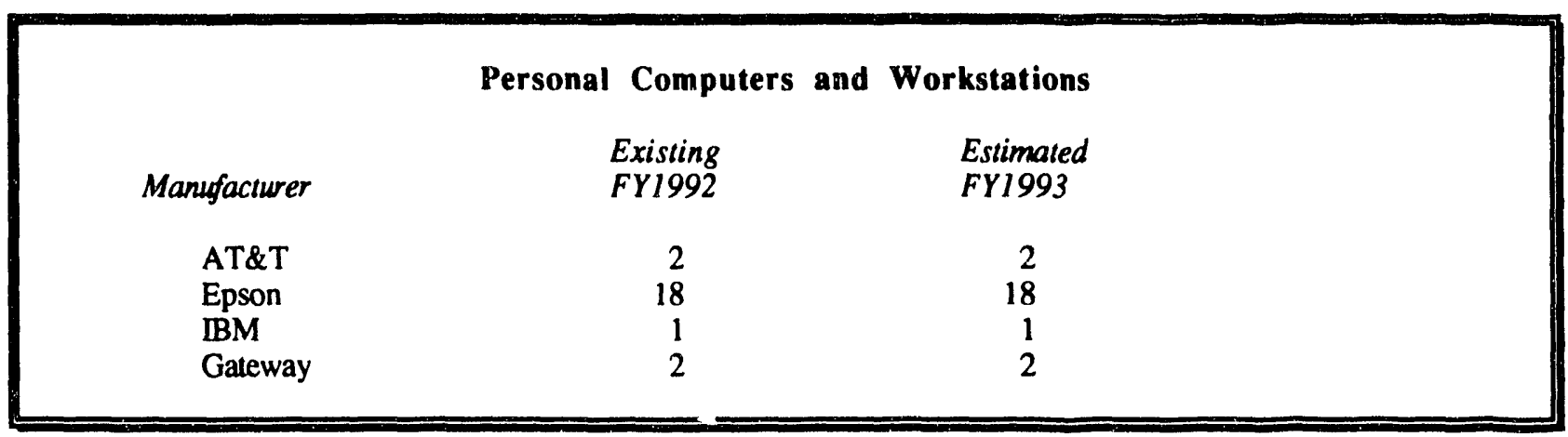

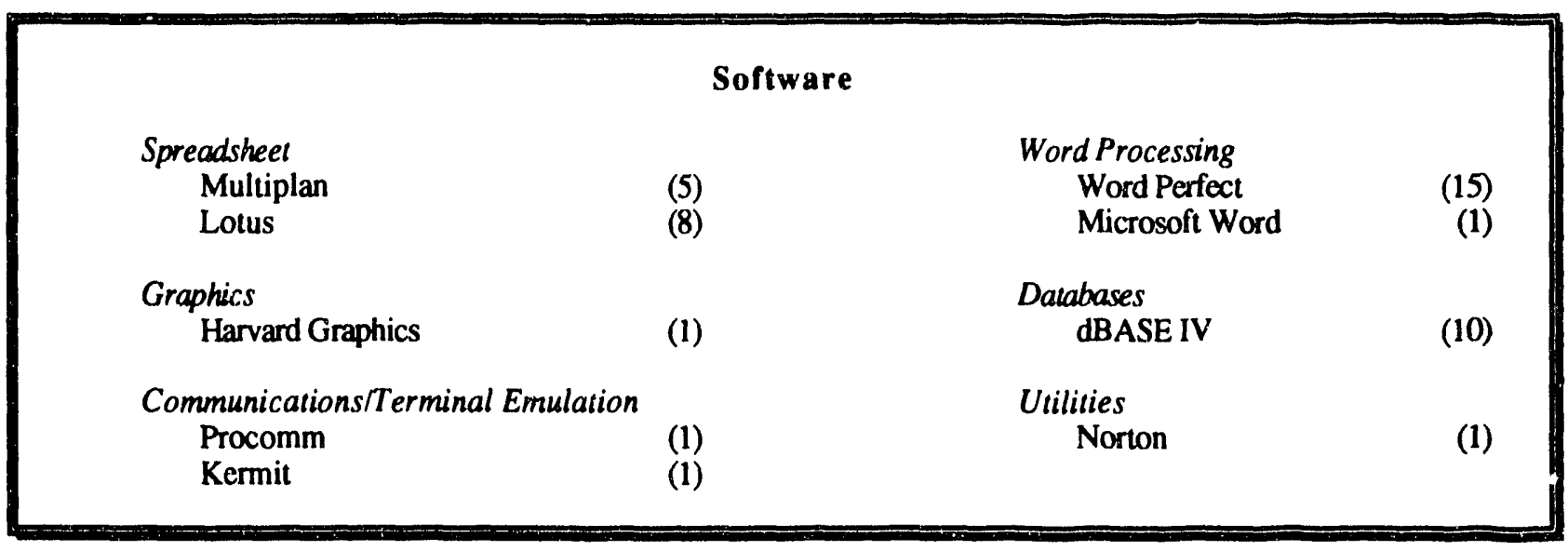


Planned activities andlor applications to be implemented during the next fiscal year in the areas of (1) scientific work stations, (2) personal computing, (3) local area networking, and (4) office automation. Please also indicate standardized software packages for your division.

1. Scientific Work Stations: None planned.

2. Personal Computing: NBI equipment replaced by PC word processors.

3. Local Area Networking: Some PC's may be connected to a network.

4. Office Automation: None planned.

5. Standardized Software: WordPerfect is the standardized word processor being used. 

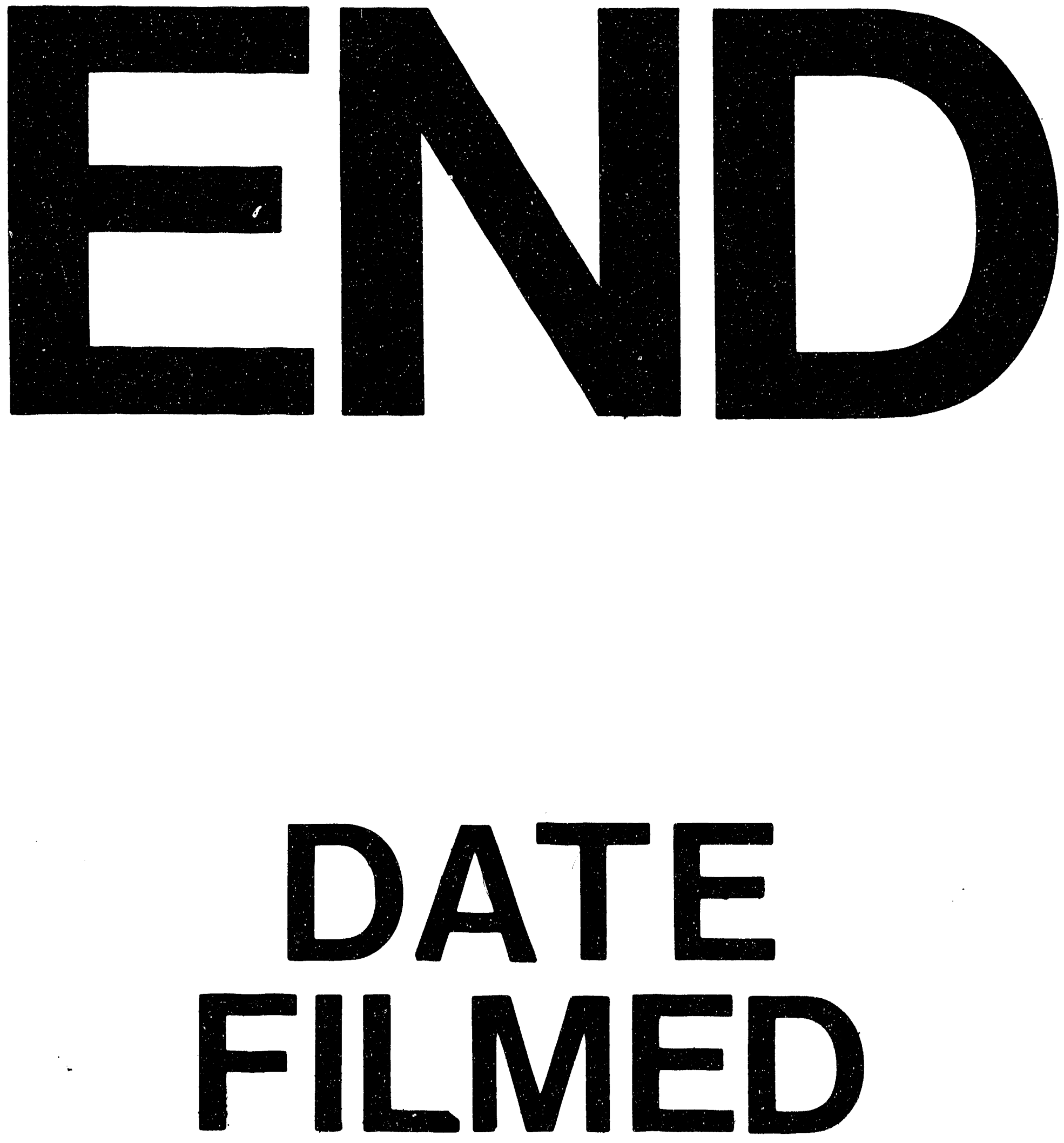

I

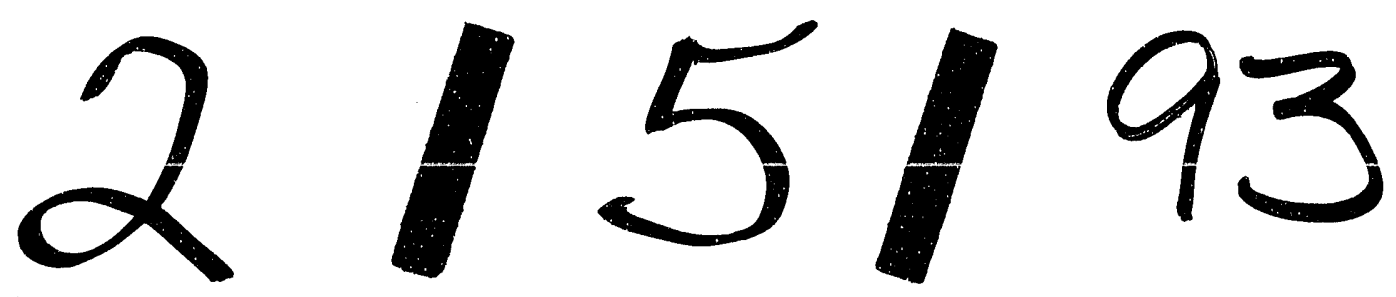


